\title{
The Meyer functions for projective varieties and their application to local signatures for fibered 4 -manifolds
}

\author{
YUSUKE KUNO
}

\begin{abstract}
We study a secondary invariant, called the Meyer function, on the fundamental group of the complement of the dual variety of a smooth projective variety. This invariant has played an important role when studying the local signatures of fibered 4-manifolds from topological point of view. As an application of our study, we define a local signature for generic nonhyperelliptic fibrations of genus 4 and 5 and compute some examples.
\end{abstract}

$14 \mathrm{D} 05,57 \mathrm{~N} 13$

\section{Introduction}

Let $\Sigma_{g}$ be a closed oriented $C^{\infty}$-surface of genus $g \geq 0$. The mapping class group of $\Sigma_{g}$, which we denote by $\Gamma_{g}$, is the group of orientation preserving diffeomorphisms of $\Sigma_{g}$ modulo isotopy. The group cohomology of $\Gamma_{g}$ attracts attentions because (1) its elements give rise to a characteristic class of oriented $\Sigma_{g}$-bundles, (2) over the rational coefficients, it is isomorphic to the cohomology of the moduli space of compact Riemann surfaces of genus $g$.

As for the degree two part, the cohomology group itself has been determined. Harer [12] proved that $H^{2}\left(\Gamma_{g} ; \mathbb{Z}\right) \cong \mathbb{Z}$ for $g \geq 3$, and the groups $H^{2}\left(\Gamma_{1} ; \mathbb{Z}\right) \cong \mathbb{Z} / 12 \mathbb{Z}$ and $H^{2}\left(\Gamma_{2} ; \mathbb{Z}\right) \cong \mathbb{Z} / 10 \mathbb{Z}$ are classically known. However, as a reflection of the fact that $\Gamma_{g}$ is related to various mathematical objects, various 2 -cocycles of $\Gamma_{g}$ are known, arising in different contexts.

One of these is Meyer's signature cocycle $\tau_{g}$, introduced by W Meyer [20] and rediscovered later by Turaev [27]. The definition involves the signature of 4-manifolds and will be recalled in this section.

The main object we study here is the Meyer function, a secondary invariant associated to $\tau_{g}$, originating in the work of Meyer [20]. He showed that for $g=1$ or 2, there exists a unique $\mathbb{Q}$-valued 1 -cochain $\phi_{g}: \Gamma_{g} \rightarrow \mathbb{Q}$ whose coboundary equals $\tau_{g}$. He also gave an explicit formula for $\phi_{1}$. Note that $\Gamma_{1}$ is isomorphic to $\operatorname{SL}(2 ; \mathbb{Z})$. Atiyah [4] showed interesting aspects of $\phi_{1}$ as a function: he showed that the value of $\phi_{1}$ for a 
hyperbolic element $\alpha \in \operatorname{SL}(2 ; \mathbb{Z})$ coincides with various values associated to $\alpha$ such as the special value of a Shimizu $L$-function determined by $\alpha$, an arithmetic invariant, or the $\eta$-invariant of the mapping torus of $\alpha$, a differential-geometric invariant.

Several recent works give higher genera or higher dimensional analogues of $\phi_{1}$ or $\phi_{2}$. Endo [8] and Morifuji [22] study the Meyer function on the hyperelliptic mapping class group. Endo [8] gives an application to the local signature for hyperelliptic fibrations and Morifuji [22] studies a relation to the $\eta$-invariant of mapping tori. Iida [14] studies, as a higher dimensional generalization of $\phi_{2}$, the Meyer function for the family of smooth theta divisors.

The Meyer function for a projective variety In this paper we give other analogues of Meyer's $\phi_{1}$ or $\phi_{2}$ and discuss their applications. We consider the family of Riemann surfaces constructed as follows.

Let $X \subset \mathbb{P}_{N}$ be a smooth complex projective variety of dimension $n$, embedded in the complex projective space of dimension $N$. Throughout the paper, we assume that

$$
N>n \geq 2 .
$$

Let $k:=N-n+1$. We denote by $G_{k}\left(\mathbb{P}_{N}\right)$ the set of all $k$-planes of $\mathbb{P}_{N}$. Let $D_{X}$ be the set of $k$-planes meeting $X$ not transversally. When $n=2, D_{X}$ is the classical dual variety of $X$. In Gel'fand, Kapranov and Zelevinsky [9], $D_{X}$ is called the $k$-th associated subvariety of $X$. Let $U^{X}=G_{k}\left(\mathbb{P}_{N}\right) \backslash D_{X}$. For $W \in U^{X}, W$ and $X$ meet transversally so their intersection $X \cap W$ has a natural structure of a compact Riemann surface. Thus setting

$$
\mathcal{C}^{X}:=\left\{(x, W) \in \mathbb{P}_{N} \times U^{X}: x \in X \cap W\right\},
$$

the second projection

$$
p_{X}:=\left.p_{2}\right|_{\mathcal{C}^{X}}: \mathcal{C}^{X} \rightarrow U^{X}
$$

is a complex analytic family of compact Riemann surfaces. Let $g$ be the genus of the fibers and

$$
\rho_{X}: \pi_{1}\left(U^{X}\right) \rightarrow \Gamma_{g}
$$

the topological monodromy (see the conventions below) of (1.0.1). Let $\rho_{X}^{*} \tau_{g}$ be the pullback of $\tau_{g}$ by $\rho_{X}$.

Theorem 1.0.2 (Theorem 3.1.1) There exists a unique $\mathbb{Q}$-valued 1-cochain $\phi_{X}: \pi_{1}\left(U^{X}\right) \rightarrow \mathbb{Q}$ whose coboundary equals $\rho_{X}^{*} \tau_{g}$. In particular, we have $\rho_{X}^{*}\left[\tau_{g}\right]=$ $0 \in H^{2}\left(\pi_{1}\left(U^{X}\right) ; \mathbb{Q}\right)$. 
We remark here that if $\rho: G \rightarrow \Gamma_{g}$ is a homomorphism from a group $G$ to $\Gamma_{g}$ and $\phi: G \rightarrow \mathbb{Q}$ is a 1 -cochain whose coboundary equals $\rho^{*} \tau_{g}$, then $\phi$ is always a class function: $\phi\left(x y x^{-1}\right)=\phi(y)$ for $x, y \in G$. This is easily derived from properties of $\tau_{g}$ (see Kuno [16, Appendix]). In particular the above $\phi_{X}$ is a class function. We call $\phi_{X}$ the Meyer function associated to $X \subset \mathbb{P}_{N}$. In fact this theorem is regarded as a further generalization of [16], where the case of $X$ being the $d$-th Veronese image of $\mathbb{P}_{2}$ is studied. Our proof is based on a geometric feature of $\tau_{g}$, and applications of the Novikov additivity of the signature are essential.

There are several studies on the fundamental group of the complement of the dual variety (or more generally the associated subvariety in a Grassmannian); for example, see Carlson and Toledo [6;7] and recent work of Shimada [26]. However, it is still a mysterious object and lots of the properties are unknown. The function $\phi_{X}$ tells us some information about $\pi_{1}\left(U^{X}\right)$ under a mild condition; see Proposition 3.6.1.

An application to local signatures One reason to seek a generalization of Meyer's $\phi_{1}$ or $\phi_{2}$ comes from the motivation to treat localization of the signature for the case of the genus of the fibers greater than two via Meyer functions.

Let us go back to the case of $g=1$ or 2 for explanation. The coboundary condition $\delta \phi_{g}=\tau_{g}$ leads to an immediate consequence: for an oriented surface bundle of genus $\leq 2$ over a closed oriented surface, the signature of the total space is zero. Proceeding further, let $M$ (resp. $B$ ) be a closed oriented $C^{\infty}$-manifold of dimension 4 (resp. 2) and $f: M \rightarrow B$ a proper surjective $C^{\infty}$-map having the structure of surface bundle of genus $g$, outside of finitely many points $b_{1}, \ldots, b_{m} \in B$. We call such a triple $(M, f, B)$ a fibered 4-manifold. The fiber germ $\mathcal{F}_{i}$ over $b_{i}$ is called a singular fiber germ. Typical examples are elliptic surfaces or Lefschetz fibrations.

In the above situation, the advantage of $\phi_{1}$ or $\phi_{2}$ is that we can associate each singular fiber germ with its local invariant $\sigma\left(\mathcal{F}_{i}\right) \in \mathbb{Q}$, called the local signature. The adjective "local" comes from the equality

$$
\operatorname{Sign}(M)=\sum_{i=1}^{m} \sigma\left(\mathcal{F}_{i}\right) .
$$

The definition of $\sigma\left(\mathcal{F}_{i}\right)$ is given by

$$
\sigma\left(\mathcal{F}_{i}\right)=\phi_{g}\left(x_{i}\right)+\operatorname{Sign}\left(N\left(f^{-1}\left(b_{i}\right)\right)\right),
$$

where $x_{i} \in \Gamma_{g}$ is the local monodromy around $b_{i}$ and $\operatorname{Sign}\left(N\left(f^{-1}\left(b_{i}\right)\right)\right)$ is the signature of a fiber neighborhood of $f^{-1}\left(b_{i}\right)$. This formulation first appeared in Y Matsumoto's papers $[18 ; 19]$. For generalizations of this story to higher genera, there is an obstruction: the class $\left[\tau_{g}\right]$ is a generator of $H^{2}\left(\Gamma_{g} ; \mathbb{Q}\right) \cong \mathbb{Q}$ for $g \geq 3$. 
Local signatures are also studied from the complex-geometric or algebro-geometric point of view; see Ashikaga and Konno [2] and Ashikaga and Yoshikawa [3]. In these settings, a local signature is defined another way and can be defined even if $g \geq 3$, by assigning some algebro-geometric conditions on the general fibers. There is an important point to note here: when $g \geq 3$, there is a fiber germ with a nontrivial local signature but which is topologically a trivial $\Sigma_{g}$-bundle. To capture such phenomena, it is insufficient just to look at the shape of $f^{-1}\left(b_{i}\right)$ or the local monodromy $x_{i}$, hence we need to modify (1.0.3).

As for higher genera analogues of Matsumoto's approach, Endo [8] studied the local signature for hyperelliptic fibrations. In [16] the author discussed nonhyperelliptic fibrations of genus 3. In this paper using the Meyer functions $\phi_{X}$ for particular choices of $X$, we will discuss nonhyperelliptic fibrations of genus 4 or 5 . The modification of (1.0.3) is achieved by introducing a group with some universal property and the Meyer function on that group. The monodromy $x_{i}$ in (1.0.3) is replaced by the lifted monodromy; see Definition 4.1.10. One advantage of our local signature is that we only need the complex structures on the general fibers so it is not necessary $f$ itself should be holomorphic. Although we don't know whether our local signature is the same as the others $[2 ; 3]$, we will observe the coincidence on some examples of singular fiber germs.

Organization of the paper Section 2 is a preparation for Section 3. We describe the tangent space of $D_{X}$ and study the situation when a holomorphic disk intersects $D_{X}$ transversally. These considerations will be used in Proposition 3.2.3. In Section 3 we prove Theorem 1.0.2 by a purely topological argument. Using the method of Lefschetz pencils, we give a formula for the value of $\phi_{X}$ on a special element, called the lasso. We also study the second bounded cohomology of $\pi_{1}\left(U^{X}\right)$. In Section 4 applications to nonhyperelliptic fibrations of genus 4 or 5 are discussed. First we explain our approach to local signatures via Meyer functions (see Proposition 4.1.8), then proceed to the particular cases. When the genus is 4 , we assign the general fibers to be "of rank 4". When the genus is 5 , we assign the general fibers to be nontrigonal. In the case of genus 4 , we compute the value of our local signature for some fiber germs.

In the rest of this introduction we fix conventions and recall Meyer's signature cocycle.

Topological monodromy We adopt the following: (1) For any two mapping classes $f_{1}$ and $f_{2}$, the multiplication $f_{1} \circ f_{2}$ means that $f_{2}$ is applied first. (2) For any two homotopy classes of based loops $\ell_{1}$ and $\ell_{2}$, their product $\ell_{1} \cdot \ell_{2}$ means that $\ell_{1}$ is traversed first. 
Let $p: \mathcal{C} \rightarrow B$ be an oriented $\Sigma_{g}$-bundle. Choose a base point $b_{0} \in B$ and fix an identification $\phi: \Sigma_{g} \cong p^{-1}\left(b_{0}\right)$. For each based loop $\ell:[0,1] \rightarrow B$ the pullback $\ell^{*} \mathcal{C} \rightarrow[0,1]$ by $\ell$ is a trivial $\Sigma_{g}$-bundle. Hence there exists a trivialization $\Phi: \Sigma_{g} \times[0,1] \rightarrow \ell^{*} \mathcal{C}$ such that $\Phi(x, 0)=\phi(x), x \in \Sigma_{g}$. By assigning the isotopy class of $\Phi(\cdot, 1)^{-1} \circ \phi$ to the homotopy class of $\ell$, we obtain a map $\rho$, called the topological monodromy of $p: \mathcal{C} \rightarrow B$, from $\pi_{1}\left(B, b_{0}\right)$ to $\Gamma_{g}$. Under the conventions above, $\rho$ is a homomorphism.

Meyer's signature cocycle Let $P$ denote the pair of pants, ie, $P=S^{2} \backslash \bigcup_{i=1}^{3}$ Int $D_{i}$ where $D_{i}, 1 \leq i \leq 3$, are the three disjoint closed disks in the 2 -sphere $S^{2}$. Choose a base point $p_{0} \in \operatorname{Int} P$ and fix based loops $\ell_{i}, 1 \leq i \leq 3$, such that each $\ell_{i}$ is homotopic to the loop traveling once the boundary $\partial D_{i}$ by counterclockwise manner, and the product $\ell_{1} \cdot \ell_{2} \cdot \ell_{3}$ is nullhomotopic. For $\left(f_{1}, f_{2}\right) \in \Gamma_{g} \times \Gamma_{g}$, we can construct an oriented $\Sigma_{g}$-bundle $E\left(f_{1}, f_{2}\right)$ over $P$ such that the topological monodromy $\pi_{1}\left(P, p_{0}\right) \rightarrow \Gamma_{g}$ sends $\left[\ell_{i}\right]$ to $f_{i}$ for $i=1,2$. The bundle $E\left(f_{1}, f_{2}\right)$ is a compact $C^{\infty}$-manifold of dimension 4 endowed with the natural orientation. Thus the signature of $E\left(f_{1}, f_{2}\right)$ is defined and we set

$$
\tau_{g}\left(f_{1}, f_{2}\right):=\operatorname{Sign}\left(E\left(f_{1}, f_{2}\right)\right) .
$$

By the Novikov additivity of the signature, $\tau_{g}$ turns out to be a 2-cocycle of $\Gamma_{g}$. The class $\left[\tau_{g}\right] \in H^{2}\left(\Gamma_{g} ; \mathbb{Z}\right)$ equals $1 / 3$ times the first MMM class; see Miller [21], Morita [23] and Mumford [25].

There is a linear-algebraic description of $\tau_{g}$ given by Meyer [20]. Let $\Gamma_{g} \rightarrow \operatorname{Sp}(2 g ; \mathbb{Z})$ be the homomorphism obtained by the action of $\Gamma_{g}$ on the first homology of $\Sigma_{g}$, and let $A_{1}$ and $A_{2}$ be the image of $f_{1}$ and $f_{2}$ by this homomorphism, respectively. Let

$$
J=\left(\begin{array}{cc}
0 & I_{g} \\
-I_{g} & 0
\end{array}\right)
$$

where $I_{g}$ is the $g \times g$ identity matrix, and consider the linear space

$$
V_{A_{1}, A_{2}}:=\left\{(x, y) \in \mathbb{R}^{2 g} \oplus \mathbb{R}^{2 g}:\left(A_{1}^{-1}-I_{2 g}\right) x+\left(A_{2}-I_{2 g}\right) y=0\right\},
$$

where $I_{2 g}$ is the $2 g \times 2 g$ identity matrix. Then

$$
\left\langle(x, y),\left(x^{\prime}, y^{\prime}\right)\right\rangle_{A_{1}, A_{2}}={ }^{t}(x+y) J\left(I_{2 g}-A_{2}\right) y^{\prime}
$$

turns out to be a symmetric bilinear form on $V_{A_{1}, A_{2}}$ hence its signature is defined. As proved in [20], we have

$$
\tau_{g}\left(f_{1}, f_{2}\right)=\operatorname{Sign}\left(V_{A_{1}, A_{2}},\langle,\rangle_{A_{1}, A_{2}}\right) .
$$


Here we correct some errors about signs in [16]. In the Appendix of [16], we have adopted the same notation about topological monodromies as this paper and have defined $\tau_{g}\left(f_{1}, f_{2}\right)=-\operatorname{Sign}\left(E\left(f_{1}, f_{2}\right)\right)$. Then Definition 7.1 on page 943 should be corrected as

$$
\operatorname{loc} . \operatorname{sig}^{\mathcal{Q}}(\mathcal{F}):=-\phi^{4}\left(\theta\left(\mathcal{F}^{0}\right)_{*}(\gamma)\right)+\operatorname{Sign}(E) .
$$

The equation $\operatorname{Sign}\left(\pi^{-1}\left(B_{0}\right)\right)=\sum_{i=1}^{n} \phi^{4}\left(\theta\left(\mathcal{F}_{i}^{0}\right)_{*}(\gamma)\right)$ in the proof of Theorem 7.2 on page 944 should be corrected by multiplying the right hand side by -1 . The proof of Proposition 5.1 on page 936 should be corrected similarly and all the values of the Meyer function in [16] should be multiplied by -1 .

Notation For a 1-cochain $\phi: G \rightarrow A$ of a group $G$ with coefficient in an abelian group $A$, its coboundary means the map $\delta \phi: G \times G \rightarrow A$ defined by

$$
\delta \phi(x, y)=\phi(x)-\phi(x y)+\phi(y) .
$$

For a complex manifold $M$, we denote by $K_{M}$ the canonical divisor of $M$. More generally, for a possibly singular variety $Y$, we denote by $\omega_{Y}$ the dualizing sheaf of $Y$. We use this notion only when $Y$ is given as a hypersurface in a complex manifold $M$. In this case $\omega_{Y}$ is an invertible sheaf on $Y$ given by the adjunction formula:

$$
\omega_{Y}=\left.\left(K_{M}+Y\right)\right|_{Y}
$$

For integers $p, q$ with $0<p<q$, we denote by $G_{p, q}$ the Grassmannian of all $p$-planes of $\mathbb{C}^{q}$. Note that $G_{k}\left(\mathbb{P}_{N}\right)$ is naturally isomorphic to $G_{k+1, N+1}$.

\section{Preliminaries from complex algebraic geometry}

In this section we describe some properties of $D_{X}$. When $n=2, D_{X}$ is an irreducible variety in $G_{N-1}\left(\mathbb{P}_{N}\right)=\mathbb{P}_{N}^{\vee}$, the dual projective space of $\mathbb{P}_{N}$, and $D_{X}$ is classically known as the dual variety of $X$. In fact, the treatment here is a generalization of the treatments in Sections 1 and 2 of K Lamotke's paper [17] to the case of general $n$. Corollary 2.2.3, Proposition 2.3.8 and Theorem 2.3.12 will be used in later sections. Let

$$
\mathcal{W}:=\left\{(x, W) \in \mathbb{P}_{N} \times G_{k}\left(\mathbb{P}_{N}\right): x \in X \cap W\right\} .
$$

Then there are two projections $p_{1}: \mathcal{W} \rightarrow X$, and

$$
p_{2}: \mathcal{W} \rightarrow G_{k}\left(\mathbb{P}_{N}\right)
$$




\subsection{Coordinate description of $p_{2}$}

In the following we give an explicit coordinate description of $p_{2}$.

Let $\left(x^{0}, W_{0}\right) \in \mathcal{W}$. By choosing appropriate homogeneous coordinates $\left[x_{0}: x_{1}: \cdots: x_{N}\right]$ of $\mathbb{P}_{N}$, we may assume that $x^{0}=[1: 0: \cdots: 0]$ and $W_{0}$ is given by $x_{k+1}=\cdots=x_{N}=0$.

We first introduce local coordinates of $\mathcal{W}$ near $\left(x^{0}, W_{0}\right)$. For $x \in X, p_{1}^{-1}(x)$ is the set of $k$-planes of $\mathbb{P}_{N}$ through $x$, which is isomorphic to $G_{k, N}$. The open set $\left\{x_{0} \neq 0\right\}$ of $\mathbb{P}_{N}$ is identified with $\mathbb{C}^{N}$ by

$$
\left[x_{0}: x_{1}: \cdots: x_{N}\right] \mapsto\left(x_{1} / x_{0}, \ldots, x_{N} / x_{0}\right) .
$$

Thus for $(x, \stackrel{\circ}{W}) \in\left(X \cap\left\{x_{0} \neq 0\right\}\right) \times G_{k, N}$, considering the affine subspace $x+\stackrel{\circ}{W} \subset$ $\mathbb{C}^{N} \cong\left\{x_{0} \neq 0\right\}$ and taking its closure in $\mathbb{P}_{N}$, we have a trivialization of $p_{1}$ over $X \cap\left\{x_{0} \neq 0\right\}$ :

$$
\left(X \cap\left\{x_{0} \neq 0\right\}\right) \times G_{k, N} \stackrel{\cong}{\longrightarrow} p_{1}^{-1}\left(X \cap\left\{x_{0} \neq 0\right\}\right) .
$$

Let

$$
e_{i}:=(0, \ldots, 1, \ldots, 0)^{t} \in \mathbb{C}^{N}
$$

for $1 \leq i \leq N$. In view of (2.1.1), $\stackrel{\circ}{W}_{0}:=W_{0} \cap\left\{x_{0} \neq 0\right\}$ is the $k$-plane of $\mathbb{C}^{N}$ spanned by $e_{1}, \ldots, e_{k}$. Let $W_{1}$ be the subspace of $\mathbb{C}^{N}$ spanned by $e_{k+1}, \ldots, e_{N}$. For $F \in \operatorname{Hom}\left(\stackrel{\circ}{W}_{0}, W_{1}\right)$ let $\stackrel{\circ}{W}(F):=\operatorname{Span}\left(f_{1}, \ldots, f_{k}\right) \in G_{k, N}$, where $f_{i}:=e_{i}+F\left(e_{i}\right)$, $1 \leq i \leq k$. By this mapping $F \mapsto \stackrel{\circ}{W}(F), \mathcal{U}:=\left\{\stackrel{\circ}{W} \in G_{k, N}: \stackrel{\circ}{W} \cap W_{1}=0\right\}$ is identified with $\operatorname{Hom}\left(\mathscr{\leftrightarrow}_{0}, W_{1}\right)$. Introducing $\left\{F_{i}^{j}\right\}$ by

$$
F\left(e_{i}\right)=\sum_{j=k+1}^{N} F_{i}^{j} e_{j}, \quad 1 \leq i \leq k,
$$

for $F \in \operatorname{Hom}\left(\stackrel{\circ}{W}_{0}, W_{1}\right)$, the set of functions $\left\{F_{i}^{j}\right\}$ serves as local coordinates of $\mathcal{U}$.

Choose a sufficiently small local coordinate neighborhood $\left(U ; t_{1}, \ldots, t_{n}\right)$ of $X$ centered at $x^{0}$ (ie, $x^{0}$ corresponds to the origin $(0, \ldots, 0)$ ) so that $U \subset X \cap\left\{x_{0} \neq 0\right\} \subset \mathbb{C}^{N}$. Then points in $U$ can be expressed as $x\left(t_{1}, \ldots, t_{n}\right)=\left(x_{1}, \ldots, x_{N}\right)$ where $x_{i}=$ $x_{i}\left(t_{1}, \ldots, t_{n}\right), 1 \leq i \leq N$ are holomorphic in $t_{1}, \ldots, t_{n}$.

In view of (2.1.2),

$$
\left(U \times \mathcal{U} ; t_{1}, \ldots, t_{n},\left\{F_{i}^{j}\right\}\right)
$$

can be used as a local coordinate neighborhood of $\mathcal{W}$ centered at $\left(x^{0}, W_{0}\right)$. 
Next we introduce local coordinates of $G_{k}\left(\mathbb{P}_{N}\right)$ near $W_{0}$. Let

$$
\widehat{e}_{i}=(0, \ldots, \stackrel{i+1}{1}, \ldots, 0)^{t} \in \mathbb{C}^{N+1},
$$

$0 \leq i \leq N$ and $\widehat{W}_{0}=\operatorname{Span}\left(\widehat{e}_{0}, \widehat{e}_{1}, \ldots, \widehat{e}_{k}\right), \widehat{W}_{1}=\operatorname{Span}\left(\widehat{e}_{k+1}, \ldots, \widehat{e}_{N}\right)$. Then by the natural isomorphism $G_{k}\left(\mathbb{P}_{N}\right) \cong G_{k+1, N+1}, W_{0}$ corresponds to $\widehat{W}_{0}$ (recall that $W_{0}$ is given by $\left.x_{k+1}=\cdots x_{N}=0\right)$. For $G \in \operatorname{Hom}\left(\widehat{W}_{0}, \widehat{W}_{1}\right)$ let $W(G):=$ $\operatorname{Span}\left(g_{0}, g_{1}, \ldots, g_{k}\right)$, where $g_{i}:=\widehat{e}_{i}+G\left(\widehat{e}_{i}\right), 0 \leq i \leq k$. By this mapping $G \mapsto W(G)$, $\mathcal{V}:=\left\{W \in G_{k+1, N+1}: W \cap \widehat{W}_{1}=0\right\}$ is identified with $\operatorname{Hom}\left(\widehat{W}_{0}, \widehat{W}_{1}\right)$. Introducing $\left\{G_{i}^{j}\right\}$ by

$$
G\left(\widehat{e}_{i}\right)=\sum_{j=k+1}^{N} G_{i}^{j} \widehat{e}_{j}, \quad 0 \leq i \leq k,
$$

for $G \in \operatorname{Hom}\left(\widehat{W}_{0}, \widehat{W}_{1}\right)$, then

$$
\left(\mathcal{V},\left\{G_{i}^{j}\right\}\right)
$$

is a local coordinate neighborhood of $G_{k+1, N+1} \cong G_{k}\left(\mathbb{P}_{N}\right)$ near $W_{0}$.

Now for $\left(t_{1}, \ldots, t_{n},\left\{F_{i}^{j}\right\}\right) \in U \times \mathcal{U}$, using the local coordinates (2.1.3) and (2.1.4), write $p_{2}\left(t_{1}, \ldots, t_{n},\left\{F_{i}^{j}\right\}\right)=G\left(t_{1}, \ldots, t_{n},\left\{F_{i}^{j}\right\}\right) \in \operatorname{Hom}\left(\widehat{W}_{0}, \widehat{W}_{1}\right)$. The closure of the affine space $x\left(t_{1}, \ldots, t_{n}\right)+\stackrel{\circ}{W}(F) \subset \mathbb{C}^{N}$ in $\mathbb{P}_{N}$ must be equal to $W\left(G\left(t_{1}, \ldots, t_{n},\left\{F_{i}^{j}\right\}\right)\right)$. On the other hand, at the level of $G_{k+1, N+1}$, the closure is a $(k+1)$-plane spanned by $\left(1, x_{1}, \ldots, x_{n}\right)^{t}$ and $\hat{f}_{1}, \ldots, \hat{f}_{k}$, where for $1 \leq i \leq k, \hat{f}_{i} \in \mathbb{C}^{N+1}$ is the image of $f_{i}$ under the inclusion $\mathbb{C}^{N} \hookrightarrow \mathbb{C}^{N+1},\left(y_{1}, \ldots, y_{N}\right) \mapsto\left(0, y_{1}, \ldots, y_{N}\right)$. From these we establish the following:

Lemma 2.1.5 (Local description of $\left.p_{2}\right)$ Let $\left(U \times \mathcal{U} ; t_{1}, \ldots, t_{n},\left\{F_{i}^{j}\right\}\right)$ and $\left(\mathcal{V},\left\{G_{i}^{j}\right\}\right)$ be the local coordinate neighborhoods of $\mathcal{W}$ and $G_{k}\left(\mathbb{P}_{N}\right)$, respectively, as above, and let $G_{i}^{j}=G_{i}^{j}\left(t_{1}, \ldots, t_{n},\left\{F_{i}^{j}\right\}\right)$ be the local coordinates of the point $W\left(G\left(t_{1}, \ldots, t_{n},\left\{F_{i}^{j}\right\}\right)\right)$. Then we have

$$
\begin{array}{ll}
G_{i}^{j}=F_{i}^{j} & \text { for } 1 \leq i \leq k, k+1 \leq j \leq N, \\
G_{0}^{j}=x_{j}-\sum_{i=1}^{k} x_{i} F_{i}^{j} & \text { for } k+1 \leq j \leq N .
\end{array}
$$

\subsection{Irreducibility of $D_{X}$}

The $k$-th associated subvariety $D_{X}$ is irreducible. Although this might be well known, here we include the proof of it together with the irreducibility of some loci related to $D_{X}$. 
Definition 2.2.1 Define the subsets of $\mathcal{W}$ as follows:

$$
\mathcal{D}:=\left\{(x, W) \in \mathcal{W}: T_{x} X+T_{x} W \neq T_{x} \mathbb{P}_{N}\right\},
$$

and for integers $i \geq 1$,

$$
\begin{aligned}
\mathcal{Y}_{i} & :=\left\{(x, W) \in \mathcal{W}: \operatorname{dim}\left(T_{x} X+T_{x} W\right)=N+1-i\right\} \\
& =\left\{(x, W) \in \mathcal{W}: \operatorname{dim}\left(T_{x} X \cap T_{x} W\right)=i\right\} .
\end{aligned}
$$

Finally, for $i \geq 1, \mathcal{D}_{i}:=\bigcup_{j \geq i} \mathcal{Y}_{j}$.

Note that $\mathcal{Y}_{i}$ is empty for $i>\max (n, k)$ and $\mathcal{Y}_{i}=\mathcal{D}_{i} \backslash \mathcal{D}_{i+1}$. Also we have $p_{2}(\mathcal{D})=$ $D_{X}$. All $\mathcal{D}_{i}$ are closed analytic subsets of $\mathcal{W}$ and we have a filtration

$$
\mathcal{D}_{1}=\mathcal{W} \supset \mathcal{D}_{2}=\mathcal{D} \supset \mathcal{D}_{3} \supset \mathcal{D}_{4} \supset \cdots \supset \mathcal{D}_{\max (n, k)}
$$

Using Lemma 2.1.5, we can verify that $\mathcal{D}$ is the set of critical points of $p_{2}: \mathcal{W} \rightarrow$ $G_{k}\left(\mathbb{P}_{N}\right)$ and $\mathcal{Y}_{i}\left(\operatorname{resp} . \mathcal{D}_{i}\right)$ is the set of points $(x, W) \in \mathcal{W}$ such that the differential $\left(p_{2}\right)_{*}: T_{(x, W)} \mathcal{W} \rightarrow T_{W} G_{k}\left(\mathbb{P}_{N}\right)$ has corank $i-1$ (resp. corank $\left.\geq i-1\right)$.

Theorem 2.2.2 (1) For each $i, \mathcal{Y}_{i}$ is a connected submanifold of $\mathcal{W}$ with codimension $i^{2}-i$ and is open and dense in $\mathcal{D}_{i}$.

(2) For each $i, \mathcal{D}_{i}$ is an irreducible analytic subset of $\mathcal{W}$ with codimension $i^{2}-i$.

Since $\operatorname{dim} \mathcal{W}=\operatorname{dim} G_{k}\left(\mathbb{P}_{N}\right)+1$ and $p_{2}$ is a proper holomorphic map, we get the following which we will use later.

Corollary 2.2.3 The set $D_{X}$ is an irreducible analytic subset of $G_{k}\left(\mathbb{P}_{N}\right)$ with codimension $\geq 1$. If the codimension of $D_{X}$ is $1, p_{2}\left(\mathcal{D}_{3}\right)$ is a proper analytic subset of $D_{X}$.

Proof of Theorem 2.2.2 Let us introduce some notation. Let $V$ be a fixed $n-$ dimensional subspace of $\mathbb{C}^{N}$ and for $i=1,2, \ldots$, let

$$
Y_{i}:=\left\{W \in G_{k, N}: \operatorname{dim}(V+W)=N+1-i\right\},
$$

and $D_{i}=\bigcup_{j \geq i} Y_{j}$. By trivializations of the pair of holomorphic vector bundles $\left(\left.T \mathbb{P}_{N}\right|_{X}, T X\right)$ on $X$, we see that $\left.p_{1}\right|_{\mathcal{D}_{i}}$ and $\left.p_{1}\right|_{\mathcal{Y}_{i}}$ are holomorphic fiber bundle with fiber isomorphic to $D_{i}$ and $Y_{i}$, respectively. Since $Y_{i}$ is open and dense in $D_{i}, \mathcal{Y}_{i}$ is also open and dense in $\mathcal{D}_{i}$. We see that $Y_{i}$ has a structure of a connected complex manifold of dimension $k n-k+i-i^{2}$. This can be seen by considering the projection $Y_{i} \rightarrow G_{i}(V), W \mapsto W \cap V\left(G_{i}(V)\right.$ is the Grassmannian of all $i$-planes of $\left.V\right)$. This shows $\mathcal{Y}_{i}$ is a connected complex manifold with the desired codimension. 
We next prove the second part. Since $\mathcal{Y}_{i}$ is contained in $\mathcal{D}_{i}$, the first part shows that the set of smooth points of $\mathcal{D}_{i}$ is connected, hence $\mathcal{D}_{i}$ is irreducible. Also we have $\operatorname{dim} \mathcal{D}_{i}=\operatorname{dim} \mathcal{Y}_{i}$. This completes the proof.

\subsection{The tangent space of $D_{X}$ for the case $D_{X}$ is a hypersurface}

In this section we describe the tangent space of $D_{X}$ at a generic point of $D_{X}$ under the assumption that the codimension of $D_{X}$ is 1 , ie, $D_{X}$ is a hypersurface of $G_{k}\left(\mathbb{P}_{N}\right)$. Then $\left.p_{2}\right|_{\mathcal{D}}: \mathcal{D} \rightarrow D_{X}$ is a dominant regular map between projective varieties of the same dimension. By Sard's lemma for varieties (see Chapter 3 of Mumford [24] for instance), there exists a proper analytic subset $E^{\prime} \subset D_{X}$ such that

(1) $E^{\prime}$ contains $S\left(D_{X}\right)$, the set of singular points of $D_{X}$,

(2) the differential $\left(\left.p_{2}\right|_{\mathcal{D}}\right)_{*}: T_{(x, W)} \mathcal{D} \rightarrow T_{W} D_{X}$ is an isomorphism for $(x, W) \in$ $\left(\left.p_{2}\right|_{\mathcal{D}}\right)^{-1}\left(D_{X} \backslash E^{\prime}\right) \backslash S(\mathcal{D})$, where $S(\mathcal{D})$ denotes the set of singular points of $\mathcal{D}$.

By Theorem 2.2.2, $\mathcal{Y}_{2}$ is contained in $\mathcal{D} \backslash S(\mathcal{D})$ so $S(\mathcal{D}) \subset \mathcal{D}_{3}$. Setting $E:=$ $E^{\prime} \cup p_{2}\left(\mathcal{D}_{3}\right)$ then this is a proper analytic subset of $D_{X}$ by Corollary 2.2.3. Now we have:

Lemma 2.3.1 Suppose that the codimension of $D_{X}$ is 1 . Then there exists a proper analytic subset $E$ of $D_{X}$ such that

(1) $E$ contains $S\left(D_{X}\right)$,

(2) $\left(\left.p_{2}\right|_{\mathcal{D}}\right)^{-1}\left(D_{X} \backslash E\right) \subset \mathcal{Y}_{2}$, in particular $\left(\left.p_{2}\right|_{\mathcal{D}}\right)^{-1}\left(D_{X} \backslash E\right)$ is contained in $\mathcal{D} \backslash S(\mathcal{D})$,

(3) the differential $\left(\left.p_{2}\right|_{\mathcal{D}}\right)_{*}$ is an isomorphism at $(x, W) \in\left(\left.p_{2}\right|_{\mathcal{D}}\right)^{-1}\left(D_{X} \backslash E\right)$.

Suppose that $\left(x^{0}, W_{0}\right) \in \mathcal{D}$ and $W_{0}=p_{2}\left(x^{0}, W_{0}\right) \in D_{X} \backslash E$. By Lemma 2.3.1, $\left(x^{0}, W_{0}\right) \in \mathcal{Y}_{2}$. Then the following relations among the subspaces of $T_{W_{0}} G_{k}\left(\mathbb{P}_{N}\right)$ hold:

$$
\left(p_{2}\right)_{*}\left(T_{\left(x^{0}, W_{0}\right)} \mathcal{W}\right) \supset\left(p_{2}\right)_{*}\left(T_{\left(x^{0}, W_{0}\right)} \mathcal{Y}_{2}\right)=\left(\left.p_{2}\right|_{\mathcal{D}}\right)_{*}\left(T_{\left(x^{0}, W_{0}\right)} \mathcal{D}\right)=T_{W_{0}} D_{X}
$$

Since $\left(x^{0}, W_{0}\right) \in \mathcal{Y}_{2},\left(p_{2}\right)_{*}\left(T_{\left(x^{0}, W_{0}\right)} \mathcal{W}\right)$ has codimension 1 in $T_{W_{0}} G_{k}\left(\mathbb{P}_{N}\right)$ (see Section 2.2). Therefore, we have

$$
T_{W_{0}} D_{X}=\left(p_{2}\right)_{*}\left(T_{\left(x^{0}, W_{0}\right)} \mathcal{W}\right) .
$$


Recall the local coordinates of $\mathcal{W}$ and $G_{k}\left(\mathbb{P}_{N}\right)$ in Section 2.1. By Lemma 2.1.5, we see that $\left(p_{2}\right)_{*}\left(T_{\left(x^{0}, W_{0}\right)} \mathcal{W}\right)$ is generated by the $n$ vectors

$$
\frac{\partial x_{k+1}}{\partial t_{i}}(0) \frac{\partial}{\partial G_{0}^{k+1}}+\ldots+\frac{\partial x_{N}}{\partial t_{i}}(0) \frac{\partial}{\partial G_{0}^{N}},
$$

$1 \leq i \leq n$, and the $k(n-1)$ vectors

$$
\frac{\partial}{\partial G_{i}^{j}}
$$

$1 \leq i \leq k, k+1 \leq j \leq N$. Let

$$
\frac{\partial \boldsymbol{x}}{\partial t_{i}}=\left(\frac{\partial x_{k+1}}{\partial t_{i}}, \ldots, \frac{\partial x_{N}}{\partial t_{i}}\right)^{t} \in \mathbb{C}^{n-1}, \quad 1 \leq i \leq n .
$$

Since $\left(x^{0}, W_{0}\right) \in \mathcal{Y}_{2}$ the rank of the matrix

$$
\left(\frac{\partial \boldsymbol{x}}{\partial t_{1}}(0), \ldots, \frac{\partial \boldsymbol{x}}{\partial t_{n}}(0)\right)
$$

is $(n-2)$. By an arrangement of indices we may assume that

$$
\frac{\partial \boldsymbol{x}}{\partial t_{i}}(0), 1 \leq i \leq n-2, \text { are linearly independent, }
$$

then the other two column vectors are in the linear span of these.

Proposition 2.3.6 Suppose that the codimension of $D_{X}$ is 1 and let $E$ be as in Lemma 2.3.1. Let $\left(x^{0}, W_{0}\right) \in \mathcal{D}$ and suppose $W_{0} \in D_{X} \backslash E$. Then under the assumption (2.3.5), the tangent space $T_{W_{0}} D_{X}$ is given by

$$
T_{W_{0}} D_{X}=\left\{\sum_{i, j} u_{i}^{j} \frac{\partial}{\partial G_{i}^{j}}: \operatorname{det}\left(\boldsymbol{u}, \frac{\partial \boldsymbol{x}}{\partial t_{1}}(0), \ldots, \frac{\partial \boldsymbol{x}}{\partial t_{n-2}}(0)\right)=0\right\} .
$$

Here, $\boldsymbol{u}=\left(u_{0}^{k+1}, \ldots, u_{0}^{N}\right)^{t} \in \mathbb{C}^{n-1}$.

Proof By the assumption (2.3.5), the right hand side of (2.3.7) is a hyperplane of $T_{W_{0}} G_{k}\left(\mathbb{P}_{N}\right)$. Also, the vectors (2.3.3) and (2.3.4) are clearly contained in the right hand side of (2.3.7). This completes the proof.

Regarding $p_{2}: \mathcal{W} \rightarrow G_{k}\left(\mathbb{P}_{\mathbb{N}}\right)$ as a family of algebraic curves, we investigate its pullback by a mapping into $G_{k}\left(\mathbb{P}_{\mathbb{N}}\right)$ which does not meet $E$ and is transverse to $D_{X}$. We first show that the total space of the pullback has the structure of a manifold. 
Proposition 2.3.8 Suppose that the codimension of $D_{X}$ is 1 and let $E$ be as in Lemma 2.3.1. Let $B$ be a $C^{\infty}$-manifold of dimension $\geq 2$ and let $\iota: B \rightarrow G_{k}\left(\mathbb{P}_{N}\right)$ be a $C^{\infty}$-map satisfying $\iota^{-1}(E)=\varnothing$ and transverse to $D_{X}$. Then, the pullback

$$
\begin{aligned}
\iota^{*} \mathcal{W} & :=\{(b,(x, W)) \in B \times \mathcal{W}: \iota(b)=W\} \\
& \cong\{(b, x) \in B \times X: x \in \iota(b)\}
\end{aligned}
$$

of $p_{2}$ by $\iota$ has the natural structure of a $C^{\infty}$-manifold as a $C^{\infty}$-submanifold of $B \times X$. Moreover, if $B$ is a complex manifold and $\iota$ is a holomorphic map, $\iota^{*} \mathcal{W}$ has the natural structure of a complex manifold as a complex submanifold of $B \times X$.

Proof We only treat the case $B$ is the small disk $\Delta:=\{z \in \mathbb{C}:|z|<\varepsilon\}, \varepsilon>0$ and $\iota$ is a holomorphic map such that $\iota^{-1}\left(D_{X}\right)=\{0\}$. A similar argument proves the general case (see also Lemma 2.4 in [16]).

By the assumption, we have $\iota(0) \in D_{X} \backslash E$ and the transversality

$$
\iota_{*}\left(T_{0} \Delta\right)+T_{\iota(0)} D_{X}=T_{\iota(0)} G_{k}\left(\mathbb{P}_{N}\right) .
$$

Let $\left(z_{0}, x^{0}\right) \in \iota^{*} \mathcal{W}$ and write $W_{0}:=\iota\left(z_{0}\right)$. Choosing the local coordinates of $\mathcal{W}$ and $G_{k}\left(\mathbb{P}_{N}\right)$ as in Section 2.1 we denote by $\iota_{i}^{j}$ the coordinate expression of $\iota$ with respect to the local coordinates $\left\{G_{i}^{j}\right\}$. In particular, we have $\iota_{i}^{j}\left(z_{0}\right)=0$. By Lemma 2.1.5, the local equation of $\iota^{*} \mathcal{W}$ near $\left(z_{0}, x^{0}\right)$ is given by

$$
-\iota_{0}^{j}(z)+x_{j}\left(t_{1}, \ldots, t_{n}\right)-\sum_{i=1}^{k} x_{i}\left(t_{1}, \ldots, t_{n}\right) \iota_{i}^{j}(z)=0
$$

for $k+1 \leq j \leq N$. Let $\psi_{j}\left(z, t_{1}, \ldots, t_{n}\right)$ be the left hand side of the above equation. The Jacobian matrix of $\left(\psi_{k+1}, \ldots, \psi_{N}\right)$ at $\left(z_{0}, 0, \ldots, 0\right)$ is the $(n-1) \times(n+1)$ matrix

$$
\left(-\iota^{\prime}\left(z_{0}\right), \frac{\partial \boldsymbol{x}}{\partial t_{1}}(0), \ldots, \frac{\partial \boldsymbol{x}}{\partial t_{n}}(0)\right),
$$

where $\iota^{\prime}=\left(d \iota_{0}^{k+1} / d z, \ldots, d \iota_{0}^{N} / d z\right)$. We claim that this matrix is of full rank. Suppose $\left(x^{0}, W_{0}\right) \notin \mathcal{D}$, namely $\left(x^{0}, W_{0}\right) \in \mathcal{Y}_{1}$. Then the $(n-1) \times n$ matrix obtained by deleting the first column of (2.3.10) is already of full rank so is (2.3.10).

Suppose $\left(x^{0}, W_{0}\right) \in \mathcal{D}$. Then by the assumption, $z_{0}=0$ and $W_{0} \in D_{X} \backslash E$. Proposition 2.3.6 and (2.3.9) shows that

$$
\operatorname{det}\left(\boldsymbol{\iota}^{\prime}(0), \frac{\partial \boldsymbol{x}}{\partial t_{1}}(0), \ldots, \frac{\partial \boldsymbol{x}}{\partial t_{n-2}}(0)\right) \neq 0,
$$

therefore (2.3.10) is of full rank also in this case. By the implicit function theorem the assertion follows. 
Let $\Delta$ and $\iota$ be as in the proof of Proposition 2.3.8. The pullback $\iota^{*} \mathcal{W}=\{(z, x) \in$ $\Delta \times X: x \in \iota(z)\}$ has the natural projection $f_{\iota}: \iota^{*} \mathcal{W} \rightarrow \Delta$. Explicitly, $f_{\iota}$ is given by $f_{\iota}(z, x)=z$.

Theorem 2.3.12 Notation is as above. Then, $\left(z_{0}, x^{0}\right) \in \iota^{*} \mathcal{W}$ is a critical point of $f_{\iota}$ if and only if $z_{0}=0$ and $\left(x^{0}, \iota(0)\right) \in \mathcal{D}$. All the critical points are nondegenerate.

In fact, we will see in Corollary 3.4.6 that there is only one critical point. By an argument like the Morse lemma, we see that near each critical point $f_{\iota}$ looks like $\left(z_{1}, z_{2}\right) \mapsto z_{1}^{2}+z_{2}^{2}$. The next section is devoted to the proof of this theorem.

\subsection{Proof of Theorem 2.3.12}

We will use the notation in the proof of Proposition 2.3.8.

Let $\left(z_{0}, x^{0}\right) \in \iota^{*} \mathcal{W}$ and write $W_{0}:=\iota\left(z_{0}\right)$. Suppose $\left(x^{0}, W_{0}\right) \notin \mathcal{D}$. Then we may assume that in the column vectors of (2.3.10)

$$
\frac{\partial \boldsymbol{x}}{\partial t_{1}}(0), \ldots, \frac{\partial \boldsymbol{x}}{\partial t_{n-1}}(0)
$$

are linearly independent. By the implicit function theorem, there exist local coordinates $\left(s_{1}, s_{2}\right)$ of $\iota^{*} \mathcal{W}$ centered at $\left(z_{0}, x^{0}\right)$ such that the points near $\left(z_{0}, x^{0}\right)$ can be expressed as $\left(z, t_{1}, \ldots, t_{n-1}, t_{n}\right)$ where

$$
z=s_{1}, t_{1}=t_{1}\left(s_{1}, s_{2}\right), \ldots, t_{n-1}=t_{n-1}\left(s_{1}, s_{2}\right), t_{n}=s_{2},
$$

and $t_{i}\left(s_{1}, s_{2}\right)$ are holomorphic in $s_{1}, s_{2}$. Since $f_{l}(z, x)=z=s_{1},\left(z_{0}, x^{0}\right)$ is not a critical point of $f_{l}$.

Suppose $\left(x^{0}, W_{0}\right) \in \mathcal{D}$. Then as we have seen in the proof of Proposition 2.3.8, $z_{0}=0$, $W_{0} \in D_{X} \backslash E$, and $\left(x^{0}, W_{0}\right) \in \mathcal{Y}_{2}$. We may assume (2.3.5). Then we have the inequality (2.3.11) hence there exist local coordinates $\left(s_{1}, s_{2}\right)$ of $\iota^{*} \mathcal{W}$ centered at $\left(z_{0}, x^{0}\right)$ such that

(2.4.1) $z=z\left(s_{1}, s_{2}\right), t_{1}=t_{1}\left(s_{1}, s_{2}\right), \ldots, t_{n-2}=t_{n-2}\left(s_{1}, s_{2}\right), t_{n-1}=s_{1}, t_{n}=s_{2}$.

For $k+1 \leq j \leq N$, differentiating the identity

$$
\psi_{j}\left(z\left(s_{1}, s_{2}\right), t_{1}\left(s_{1}, s_{2}\right), \ldots, t_{n-2}\left(s_{1}, s_{2}\right), s_{1}, s_{2}\right)=0
$$

with respect to $s_{1}$ and setting $\left(s_{1}, s_{2}\right)=(0,0)$, we have

$$
-\frac{\partial z}{\partial s_{1}}(0) \iota^{\prime}(0)+\sum_{i=1}^{n-2} \frac{\partial t_{i}}{\partial s_{1}}(0) \frac{\partial \boldsymbol{x}}{\partial t_{i}}(0)+\frac{\partial \boldsymbol{x}}{\partial t_{n-1}}(0)=0 .
$$


But by the transversality, $\iota^{\prime}(0)$ is not contained in $\operatorname{Span}\left(\partial \boldsymbol{x} / \partial t_{i}(0)\right)_{1 \leq i \leq n}$. Thus we have $\partial z / \partial s_{1}(0)=0$ and the identity

$$
\sum_{i=1}^{n-2} \frac{\partial t_{i}}{\partial s_{1}}(0) \frac{\partial \boldsymbol{x}}{\partial t_{i}}(0)+\frac{\partial \boldsymbol{x}}{\partial t_{n-1}}(0)=0 .
$$

Similarly, we have $\partial z / \partial s_{2}(0)=0$ and the identity

$$
\sum_{i=1}^{n-2} \frac{\partial t_{i}}{\partial s_{2}}(0) \frac{\partial \boldsymbol{x}}{\partial t_{i}}(0)+\frac{\partial \boldsymbol{x}}{\partial t_{n}}(0)=0
$$

This shows that $\left(0, x^{0}\right)$ is a critical point of $f_{l}$. We have proved the first part.

To accomplish the proof, we must show that all the critical points are nondegenerate. We need to compute the Hessian of $z\left(s_{1}, s_{2}\right)$ at $\left(s_{1}, s_{2}\right)=(0,0)$ where $z\left(s_{1}, s_{2}\right)$ is as in (2.4.1). For this purpose we give a system of local equations for the submanifold $\mathcal{Y}_{2}$, and we rephrase the fact that the differential $\left(\left.p_{2}\right|_{\mathcal{D}}\right)_{*}: T_{\left(x^{0}, W_{0}\right)} \mathcal{D}=T_{\left(x^{0}, W_{0}\right)} \mathcal{Y}_{2} \rightarrow T_{W_{0}} D_{X}$ is an isomorphism.

Now take the local coordinates $(2.1 .3)$ of $\mathcal{W}$. Then for $(x, W)$ in this coordinate neighborhood, $T_{x} X=T_{x}\left(X \cap \mathcal{U}_{0}\right) \subset \mathbb{C}^{N}$ is spanned by the $n$ vectors

$$
\alpha_{i}=\left(\frac{\partial x_{1}}{\partial t_{i}}, \ldots, \frac{\partial x_{N}}{\partial t_{i}}\right)^{t}, \quad 1 \leq i \leq n,
$$

and $T_{x} W=T_{x}\left(W \cap \mathcal{U}_{0}\right)$ is spanned by the $k$ vectors

$$
\beta_{i}=\left(0, \ldots, 1, \ldots, 0, F_{i}^{k+1}, \ldots, F_{i}^{N}\right)^{t}, \quad 1 \leq i \leq k .
$$

$(x, W) \in \mathcal{Y}_{2}$ if and only if the linear span of these $n+k=N+1$ vectors is $(N-1)$-dimensional. On the other hand, the origin $\left(x^{0}, W_{0}\right)$ is in $\mathcal{Y}_{2}$ and by the assumption (2.3.5) $\alpha_{1}, \ldots, \alpha_{n-2}, \beta_{1}, \ldots, \beta_{k}$ are linearly independent at $\left(x^{0}, W_{0}\right)$. Therefore, the vanishing of the two determinants $\operatorname{det}\left(\alpha_{1}, \ldots, \alpha_{n-2}, \alpha_{n-1}, \beta_{1}, \ldots, \beta_{k}\right)$ and $\operatorname{det}\left(\alpha_{1}, \ldots, \alpha_{n-2}, \alpha_{n}, \beta_{1}, \ldots, \beta_{k}\right)$ gives a system of local equations for $\mathcal{Y}_{2}$ near $\left(x^{0}, W_{0}\right)$. By elementary transformations of matrices, we see that these determinants are equal up to sign to $\Phi_{n-1}$ and $\Phi_{n}$ respectively, where

$$
\Phi_{v}=\operatorname{det}\left(\frac{\partial \boldsymbol{x}}{\partial t_{1}}-\sum_{i=1}^{k} \frac{\partial x_{i}}{\partial t_{1}} \boldsymbol{F}_{i}, \cdots, \frac{\partial \boldsymbol{x}}{\partial t_{n-2}}-\sum_{i=1}^{k} \frac{\partial x_{i}}{\partial t_{n-2}} \boldsymbol{F}_{i}, \frac{\partial \boldsymbol{x}}{\partial t_{v}}-\sum_{i=1}^{k} \frac{\partial x_{i}}{\partial t_{v}} \boldsymbol{F}_{i}\right) .
$$

Here, $\boldsymbol{F}_{i}=\left(F_{i}^{k+1}, \ldots, F_{i}^{N}\right)^{t}$. Hence $\mathcal{Y}_{2}$ is locally given by $\Phi_{n-1}=\Phi_{n}=0$.

Now the fact that $\left(\left.p_{2}\right|_{\mathcal{D}}\right)_{*}: T_{\left(x^{0}, W_{0}\right)} \mathcal{Y}_{2} \rightarrow T_{W_{0}} D_{X}$ is an isomorphism can be rephrased as follows: the rank of the Jacobian matrix of $\left(\Phi_{n-1}, \Phi_{n}, p_{2}\right)$ at $\left(x^{0}, W_{0}\right)$ is equal 
to $\operatorname{dim} D_{X}+2=(k+1)(n-1)+1$. Again by elementary transformations, this is equivalent to the following:

Lemma 2.4.4 Let $\left(x^{0}, \mathcal{W}_{0}\right)$ and let $\Phi_{n-1}$ and $\Phi_{n}$ be as in the above. Then the rank of the $(n+1) \times n$ matrix

$$
\left(\begin{array}{ccc}
\frac{\partial \Phi_{n-1}}{\partial t_{1}}(0) & \cdots & \frac{\partial \Phi_{n-1}}{\partial t_{n}}(0) \\
\frac{\partial \Phi_{n}}{\partial t_{1}}(0) & \cdots & \frac{\partial \Phi_{n}}{\partial t_{n}}(0) \\
\frac{\partial \boldsymbol{x}}{\partial t_{1}}(0) & \cdots & \frac{\partial \boldsymbol{x}}{\partial t_{n}}(0)
\end{array}\right)
$$

is equal to $(k+1)(n-1)+1-k(n-1)=n$.

We perform the following two elementary transformation to (2.4.5): let $C_{i}$ be the $i$-th column of (2.4.5), then (1) add $\sum_{i=1}^{n-2} \partial t_{i} / \partial s_{1}(0) C_{i}$ to the (n-1)-th column, and (2) add $\sum_{i=1}^{n-2} \partial t_{i} / \partial s_{2}(0) C_{i}$ to the $n$-th column. Then by (2.4.2) and (2.4.3), (2.4.5) is transformed into

$$
\left(\begin{array}{ccccc}
\frac{\partial \Phi_{n-1}}{\partial t_{1}}(0) & \cdots & \frac{\partial \Phi_{n-1}}{\partial t_{n-2}}(0) & A_{11} & A_{12} \\
\frac{\partial \Phi_{n}}{\partial t_{1}}(0) & \cdots & \frac{\partial \Phi_{n}}{\partial t_{n-2}}(0) & A_{21} & A_{22} \\
\frac{\partial \boldsymbol{x}}{\partial t_{1}}(0) & \cdots & \frac{\partial \boldsymbol{x}}{\partial t_{n-2}}(0) & \mathbf{0} & \mathbf{0}
\end{array}\right)
$$

where

$$
A_{\lambda \mu}=\sum_{i=1}^{n-2} \frac{\partial t_{i}}{\partial s_{\mu}}(0) \frac{\partial \Phi_{n+\lambda-2}}{\partial t_{i}}(0)+\frac{\partial \Phi_{n+\lambda-2}}{\partial t_{n+\mu-2}}(0) .
$$

Now combining Lemma 2.4.4 and (2.3.5), we see that

$$
\operatorname{det}\left(\begin{array}{ll}
A_{11} & A_{12} \\
A_{21} & A_{22}
\end{array}\right) \neq 0 \text {. }
$$

Lemma 2.4.8 We have the equality

$$
\left(\begin{array}{ll}
A_{11} & A_{12} \\
A_{21} & A_{22}
\end{array}\right)=A_{0}\left(\begin{array}{cc}
\frac{\partial^{2} z}{\partial s_{1}^{2}}(0) & \frac{\partial^{2} z}{\partial s_{2} \partial s_{1}}(0) \\
\frac{\partial^{2} z}{\partial s_{1} \partial s_{2}}(0) & \frac{\partial^{2} z}{\partial s_{2}{ }^{2}}(0)
\end{array}\right),
$$

where $A_{0}=\operatorname{det}\left(\partial \boldsymbol{x} / \partial t_{1}(0), \ldots, \partial \boldsymbol{x} / \partial t_{n-2}(0), \boldsymbol{\iota}^{\prime}(0)\right)$. 
By (2.3.11), (2.4.7) and Lemma 2.4.8, it follows that the Hessian of $z\left(s_{1}, s_{2}\right)$ at $\left(s_{1}, s_{2}\right)=(0,0)$ is nonzero. Thus, $\left(0, x^{0}\right)$ is a nondegenerate critical point of $f_{l}$. This completes the proof of Theorem 2.3.12, modulo Lemma 2.4.8.

Lemma 2.4 .8 can be proved by a straightforward computation. We only give an outline of the proof of $A_{11}=A_{0} \partial^{2} z / \partial s_{1}{ }^{2}(0)$. The first claim is that

(2.4.9) $\frac{\partial \Phi_{n-1}}{\partial t_{i}}(0)$

$=\operatorname{det}\left(\frac{\partial \boldsymbol{x}}{\partial t_{1}}(0), \ldots, \frac{\partial \boldsymbol{x}}{\partial t_{n-2}}(0), \sum_{\ell=1}^{n-2} \frac{\partial t_{\ell}}{\partial s_{1}}(0) \frac{\partial^{2} \boldsymbol{x}}{\partial t_{i} \partial t_{\ell}}(0)+\frac{\partial^{2} \boldsymbol{x}}{\partial t_{i} \partial t_{n-1}}(0)\right)$,

which can be proved by using (2.4.2).

For $k+1 \leq j \leq N$, differentiating twice the identity

$$
\psi_{j}\left(z\left(s_{1}, s_{2}\right), t_{1}\left(s_{1}, s_{2}\right), \ldots, t_{n-2}\left(s_{1}, s_{2}\right), s_{1}, s_{2}\right)=0
$$

with respect to $s_{1}$ and setting $\left(s_{1}, s_{2}\right)=(0,0)$, we have

$$
\begin{aligned}
-\frac{d \iota_{0}^{j}}{d z}(0) & \frac{\partial^{2} z}{\partial s_{1}{ }^{2}}(0) \\
& +\sum_{i=1}^{n}\left(\left(\sum_{h=1}^{n} \frac{\partial^{2} x_{j}}{\partial t_{h} \partial t_{i}}(0) \frac{\partial t_{h}}{\partial s_{1}}(0)\right) \frac{\partial t_{i}}{\partial s_{1}}(0)+\frac{\partial x_{j}}{\partial t_{i}}(0) \frac{\partial^{2} t_{i}}{\partial s_{1}{ }^{2}}(0)\right)=0
\end{aligned}
$$

(note that $\partial z / \partial s_{1}(0)=\partial z / \partial s_{2}(0)=0, \partial t_{n-1} / \partial s_{1}(0)=1$, and $\left.\partial t_{n} / \partial s_{1}(0)=0\right)$.

Using (2.4.6), (2.4.9) and (2.4.10), we can get the desired formula.

\section{The Meyer function for a projective variety}

\subsection{Main theorem}

Recall the situation arising from $X \subset \mathbb{P}_{N}$ as described in Section 1. We focus on the topological monodromy

$$
\rho_{X}: \pi_{1}\left(U^{X}\right) \rightarrow \Gamma_{g}
$$

of (1.0.1).

Theorem 3.1.1 There exists a uniquely determined $\mathbb{Q}$-valued 1-cochain $\phi_{X}: \pi_{1}\left(U^{X}\right) \rightarrow \mathbb{Q}$ whose coboundary equals $\rho_{X}^{*} \tau_{g}$. In particular, we have $\rho_{X}^{*}\left[\tau_{g}\right]=$ $0 \in H^{2}\left(\pi_{1}\left(U^{X}\right) ; \mathbb{Q}\right)$. 
Here we comment about the group $\pi_{1}\left(U^{X}\right)$. If the codimension of $D_{X}$ is $\geq 2, \pi_{1}\left(U^{X}\right)$ is trivial since $G_{k}\left(\mathbb{P}_{N}\right)$ is simply connected. Suppose the codimension of $D_{X}$ is 1 . Then $\pi_{1}\left(U^{X}\right)$ is finitely presentable since $U^{X}$ is an affine algebraic variety, and the first Betti number $b_{1}\left(\pi_{1}\left(U^{X}\right)\right.$ ) is zero (see Lemma 3.3.1). Moreover, $\pi_{1}\left(U^{X}\right)$ is normally generated by a single element, called a lasso. Roughly speaking, a lasso is an element of $\pi_{1}\left(U^{X}\right)$ going once around $D_{X}$. The precise definition is as follows. We fix some base point in $U^{X}$. Let $W_{0}$ be a smooth point of $D_{X}$ and $\left(z_{1}, \ldots, z_{m}\right)$ be local coordinates of $G_{k}\left(\mathbb{P}_{N}\right)$ centered at $W_{0}$, such that $D_{X}$ is locally given by $z_{1}=0$. For a sufficiently small $\varepsilon>0$, consider the loop

$$
[0,1] \rightarrow U^{X}, t \mapsto\left(\varepsilon e^{2 \pi \sqrt{-1} t}, 0, \ldots, 0\right)
$$

defined in this coordinate neighborhood. Joining this loop with a path in $U^{X}$ from the base point of $U^{X}$ to $(\varepsilon, 0, \ldots, 0)$, we get an element of $\pi_{1}\left(U^{X}\right)$, which is called a lasso around $D_{X}$. The irreducibility of $D_{X}$ implies that all lassos are conjugate to each other and $\pi_{1}\left(G_{k}\left(\mathbb{P}_{N}\right)\right)=1$ implies that $\pi_{1}\left(U^{X}\right)$ is normally generated by a lasso. Since $\phi_{X}$ is a class function (see Section 1), the value of $\phi_{X}$ on any lasso is constant. This value can be computed from various invariants of $X$. For details, see Section 3.5. Nevertheless, the values of $\phi_{X}$ on an element other than lasso seems difficult to know.

The proof of Theorem 3.1.1 will be given in the next two sections. The following argument is a generalization of Sections 3 and 4 of [16].

\subsection{Proof of the existence}

It suffices to consider the case when the codimension of $D_{X}$ is 1 . The existence of $\phi_{X}$ is equivalent to $\rho_{X}^{*}\left[\tau_{g}\right]=0 \in H^{2}\left(\pi_{1}\left(U^{X}\right) ; \mathbb{Q}\right)$.

We first embed $H^{2}\left(\pi_{1}\left(U^{X}\right) ; \mathbb{Q}\right)$ into another space. Let $V_{k+1, N+1}$ be the (complex) Stiefel manifold of all $(k+1)$-frames of $\mathbb{C}^{N+1}$. Regarding $\mathbb{P}_{N}$ as the projectivization of $\mathbb{C}^{N+1}$ we have the natural projection $q: V_{k+1, N+1} \rightarrow G_{k}\left(\mathbb{P}_{N}\right)$, which is a principal $\mathrm{GL}(k+1 ; \mathbb{C})$ bundle. Let $\widetilde{D}_{X}=q^{-1}\left(D_{X}\right), \widetilde{U}^{X}=V_{k+1, N+1} \backslash \widetilde{D}_{X}$, and $\widetilde{E}=q^{-1}(E)$. For simplicity we use the same letter $q$ for the restriction $\left.q\right|_{\tilde{U}^{X}}: \widetilde{U}^{X} \rightarrow U^{X}$.

We have the following short exact sequence with rational coefficients:

$$
H^{0}\left(U^{X}\right) \stackrel{\cup c_{1}}{\longrightarrow} H^{2}\left(U^{X}\right) \stackrel{q^{*}}{\longrightarrow} H^{2}\left(\tilde{U}^{X}\right) .
$$

This is derived from the 5-term exact sequence of the principal bundle $q: \tilde{U}^{X} \rightarrow U^{X}$. Here, $c_{1}$ is the first Chern class, which is the restriction of a generator of $H^{2}\left(G_{k}\left(\mathbb{P}_{N}\right)\right)$ to $H^{2}\left(U^{X}\right)$. But since $D_{X}$ is of codimension 1 , the first Chern class $c_{1}\left(\left[D_{X}\right]\right) \in$ $H^{2}\left(G_{k}\left(\mathbb{P}_{N}\right)\right)$ is defined and is also a generator of $H^{2}\left(G_{k}\left(\mathbb{P}_{N}\right)\right)$. The point here is that 
$H^{2}\left(G_{k}\left(\mathbb{P}_{N}\right)\right)$ is of rank 1. Clearly the restriction of $c_{1}\left(\left[D_{X}\right]\right)$ to $U^{X}=G_{k}\left(\mathbb{P}_{N}\right) \backslash D_{X}$ is zero, therefore $c_{1}$ is also zero. Thus, we have the injective homomorphism

$$
q^{*}: H^{2}\left(U^{X} ; \mathbb{Q}\right) \hookrightarrow H^{2}\left(\tilde{U}^{X} ; \mathbb{Q}\right) .
$$

Let $\chi: \pi_{1}\left(\tilde{U}^{X}\right) \rightarrow \pi_{1}\left(U^{X}\right)$ be the homomorphism between fundamental groups induced by $q$. Since for any space $X$ there is the natural injection $H^{2}\left(\pi_{1}(X)\right) \rightarrow$ $H^{2}(X)$ of the second cohomology with arbitrary coefficients, (3.2.1) implies that we have the injective homomorphism

$$
\chi^{*}: H^{2}\left(\pi_{1}\left(U^{X}\right) ; \mathbb{Q}\right) \hookrightarrow H^{2}\left(\pi_{1}\left(\tilde{U}^{X}\right) ; \mathbb{Q}\right)
$$

induced by $\chi$.

Next we show $\chi^{*} \rho_{X}^{*}\left[\tau_{g}\right]=0 \in H^{2}\left(\pi_{1}\left(\tilde{U}^{X}\right) ; \mathbb{Z}\right)$. Let

$$
\widetilde{\mathcal{W}}:=\left\{(x, \widetilde{W}) \in \mathbb{P}_{N} \times V_{k+1, N+1}: x \in X \cap q(\widetilde{W})\right\}
$$

and $\tilde{p}_{2}: \widetilde{\mathcal{W}} \rightarrow V_{k+1, N+1}$ be the second projection, and

$$
\widetilde{\mathcal{C}^{X}}:=\left\{(x, \widetilde{W}) \in \mathbb{P}_{N} \times \widetilde{U}^{X}: x \in X \cap q(\widetilde{W})\right\} .
$$

The second projection $\tilde{p}_{X}: \widetilde{\mathcal{C}^{X}} \rightarrow \widetilde{U}^{X}$ is a family of Riemann surfaces, which is the pullback of $p_{X}: \mathcal{C}^{X} \rightarrow U^{X}$ by $q$. The associated topological monodromy is $\tilde{\rho}_{X}:=\rho_{X} \circ \chi$.

We construct a $1-$ cochain $c: \pi_{1}\left(\tilde{U}^{X}\right) \rightarrow \mathbb{Z}$ whose coboundary $\delta c$ coincides with $\tilde{\rho}_{X}^{*} \tau_{g}$. The point here is $V_{k+1, N+1} \backslash \widetilde{E}$ is 2 -connected. This follows from the two facts: (1) the Stiefel manifold $V_{k+1, N+1}$ is $2(N-k)$-connected and $2(N-k)=2 n-2 \geq 2$, and (2) the complex codimension of $\widetilde{E} \subset V_{k+1, N+1}$ is $\geq 2$ (see Lemma 2.3.1). All of the spaces that we consider in the rest of this section as well as all of the maps are based, otherwise stated.

Construction of $c$ Let $\ell: S^{1} \rightarrow \widetilde{U}^{X}$ be a $C^{\infty}$-loop, ie, a $C^{\infty}$-map from $S^{1}$ to $\tilde{U}^{X}$. Since $V_{k+1, N+1} \backslash \tilde{E}$ is simply connected we can extend $\ell$ to a $C^{\infty}$-map $\widetilde{\ell}: D^{2} \rightarrow V_{k+1, N+1} \backslash \widetilde{E}$ which is transverse to $\widetilde{D}_{X}$. Here we make the identifications $S^{1}=\{z \in \mathbb{C}:|z|=1\}$ and $D^{2}=\{z \in \mathbb{C}:|z| \leq 1\}$, and endow them the usual orientation: the orientation of $D^{2}$ is induced by that of $\mathbb{C}$ and $S^{1}$ goes around $D^{2}$ by counterclockwise manner.

By Proposition 2.3.8 the pullback $\widetilde{\ell^{*}} \widetilde{\mathcal{W}}:=(q \circ \tilde{\ell})^{*} \mathcal{W}$ has the natural structure of a compact oriented 4 -dimensional $C^{\infty}$-manifold with boundary. The orientation is 
induced by the orientation of $D^{2}$ and that of the general fibers of $\widetilde{\ell^{*}} \widetilde{\mathcal{W}}$, which have the natural orientations as compact Riemann surfaces. Set

$$
c([\ell]):=\operatorname{Sign}\left(\widetilde{\ell}^{*} \widetilde{\mathcal{W}}\right) \in \mathbb{Z} .
$$

Here $[\ell] \in \pi_{1}\left(\tilde{U}^{X}\right)$ is the element represented by $\ell$, and the right hand side is the signature of $\widetilde{\ell} \widetilde{\mathcal{W}}$.

Proposition 3.2.3 The above definition of $c$ is well defined. The 1 -cochain $c$ is a class function on $\pi_{1}\left(\tilde{U}^{X}\right)$ and $c\left(x^{-1}\right)=-c(x)$ for $x \in \pi_{1}\left(\tilde{U}^{X}\right)$. We have $\delta c=-\tilde{\rho}_{X}^{*} \tau_{g}$.

Proof Let $\ell_{0}$ and $\ell_{1}$ be $C^{\infty}$-loops in $\tilde{U}^{X}$. Suppose that the elements of $\pi_{1}\left(\tilde{U}^{X}\right)$ represented by them are conjugate to each other. Then there exists a $C^{\infty}$-homotopy $H: S^{1} \times[0,1] \rightarrow \widetilde{U}^{X}$ such that $H(\cdot, 0)=\ell_{0}$ and $H(\cdot, 1)=\ell_{1}$ (caution: we do not require that $H(\cdot, t)$ is a base preserving map for every $t \in[0,1])$. Identify the 2-sphere $S^{2}$ as

$$
S^{2} \cong\left(S^{1} \times[0,1]\right) \cup\left(D^{2} \times\{0\}\right) \cup\left(D^{2} \times\{1\}\right)
$$

and take some extensions $\tilde{\ell}_{i}: D^{2} \times\{i\} \rightarrow V_{k+1, N+1} \backslash \widetilde{E}$ of $\ell_{i}$ for $i=0,1$. Then piecing $H, \tilde{\ell}_{0}$, and $\tilde{\ell}_{1}$ together, we can construct a $C^{\infty}$-map $\tilde{H}: S^{2} \rightarrow V_{k+1, N+1} \backslash \widetilde{E}$ which is transverse to $\widetilde{D}_{X}$. Introduce the orientation of $S^{2}$ such that $D^{2} \times\{0\} \hookrightarrow S^{2}$ is orientation preserving. Then $D^{2} \times\{1\} \hookrightarrow S^{2}$ is orientation reversing and the pullback $\widetilde{H}^{*} \widetilde{\mathcal{W}}$ is a closed oriented 4-dimensional $C^{\infty}$-manifold. Moreover, since $\pi_{2}\left(V_{k+1, N+1} \backslash \tilde{E}\right)=0, \tilde{H}$ extends to a $C^{\infty}$-map from the 3-ball to $V_{k+1, N+1} \backslash \widetilde{E}$ which is transverse to $\widetilde{D}_{X}$. Hence $\widetilde{H}^{*} \widetilde{\mathcal{W}}$ is the boundary of a 5-dimensional manifold and the signature of $\widetilde{H}^{*} \widetilde{\mathcal{W}}$ is zero. Now by the Novikov additivity of the signature we have

$$
0=\operatorname{Sign}\left(\tilde{H}^{*} \widetilde{\mathcal{W}}\right)=\operatorname{Sign}\left(\tilde{\ell}_{0}^{*} \widetilde{\mathcal{W}}\right)-\operatorname{Sign}\left(\tilde{\ell}_{1}^{*} \widetilde{\mathcal{W}}\right)
$$

This proves that $c$ is well defined and $c$ is a class function, ie, $c\left(x y x^{-1}\right)=c(y)$ for $x, y \in \pi_{1}\left(\tilde{U}^{X}\right)$. Since changing the orientation of a manifold changes the sign of its signature, the property $c\left(x^{-1}\right)=-c(x)$ is clear.

We next prove that $\delta c=-\tilde{\rho}_{X}^{*} \tau_{g}$, ie,

$$
c\left(\left[\ell_{0}\right]\right)+c\left(\left[\ell_{1}\right]\right)-c\left(\left[\ell_{0}\right]\left[\ell_{1}\right]\right)=-\tilde{\rho}_{X}^{*} \tau_{g}\left(\left[\ell_{0}\right],\left[\ell_{1}\right]\right)
$$

for any based $C^{\infty}$-loops $\ell_{0}$ and $\ell_{1}$. Let $D_{i}, 0 \leq i \leq 2$, be embedded three disjoint closed 2-disks in $S^{2}$ and we denote its boundary circle by $S_{i}^{1}$. Let $P:=$ $S^{2} \backslash \bigsqcup_{i=0}^{2} \operatorname{Int}\left(D_{i}\right)$. Since $P$ has the homotopy type of the bouquet $S^{1} \vee S^{1}$, we can construct a $C^{\infty}$-map $L: P \rightarrow \widetilde{U}^{X}$ such that the restriction of $L$ to $S_{i}^{1} \cong S^{1}$ is equal to $\ell_{i}$ for $i=0,1$. Then the restriction of $L$ to $S_{2}^{1}$ is homotopic to the inverse 
of the composition loop $\ell_{0} \cdot \ell_{1}$. Notice that $\operatorname{Sign}\left(L^{*} \widetilde{\mathcal{W}}\right)$ is equal to $\widetilde{\rho}_{X}^{*} \tau_{g}\left(\left[\ell_{0}\right],\left[\ell_{1}\right]\right)$. Take some extensions $\tilde{\ell}_{0}, \tilde{\ell}_{1}$, and $\widetilde{\ell_{0} \cdot \ell_{1}}$ of $\ell_{0}, \ell_{1}$, and $\ell_{0} \cdot \ell_{1}$, respectively. Then by piecing them and $L$ together we have a $C^{\infty}$-map $\widetilde{L}: S^{2} \rightarrow V_{k+1, N+1} \backslash \widetilde{E}$ which is transverse to $D_{X}$. Again, the vanishing of $\pi_{2}\left(V_{k+1, N+1} \backslash \widetilde{E}\right)$ implies that the signature of $\widetilde{L}^{*} \widetilde{\mathcal{W}}$ is zero. Finally, by the Novikov additivity we have

$$
0=\operatorname{Sign}\left(\tilde{L}^{*} \widetilde{\mathcal{W}}\right)=\operatorname{Sign}\left(\tilde{\ell}_{0}^{*} \widetilde{\mathcal{W}}\right)+\operatorname{Sign}\left(\tilde{\ell}_{0}^{*} \widetilde{\mathcal{W}}\right)-\operatorname{Sign}\left(\widetilde{\ell_{0} \cdot \ell_{1}} * \widetilde{\mathcal{W}}\right)+\operatorname{Sign}\left(L^{*} \widetilde{\mathcal{W}}\right)
$$

but this equation is equivalent to (3.2.4). This completes the proof.

By Proposition 3.2.3, we have $\chi^{*} \rho_{X}^{*}\left[\tau_{g}\right]=0 \in H^{2}\left(\pi_{1}\left(\tilde{U}^{X}\right) ; \mathbb{Z}\right)$. Combining this with the injection (3.2.2) we have $\rho_{X}^{*}\left[\tau_{g}\right]=0 \in H^{2}\left(\pi_{1}\left(U^{X}\right)\right.$; $\left.\mathbb{Q}\right)$. This completes the proof of the existence of $\phi_{X}$.

\subsection{Proof of the uniqueness}

The uniqueness of $\phi_{X}$ follows from the following:

Lemma 3.3.1 The first cohomology group of $\pi_{1}\left(U^{X}\right)$ is trivial over rationals:

$$
H^{1}\left(\pi_{1}\left(U^{X}\right) ; \mathbb{Q}\right)=\operatorname{Hom}\left(\pi_{1}\left(U^{X}\right), \mathbb{Q}\right)=0 .
$$

Proof It suffices to consider the case when the codimension of $D_{X}$ is 1 . Consider the following commutative diagram among (co)homology groups with integer coefficients:

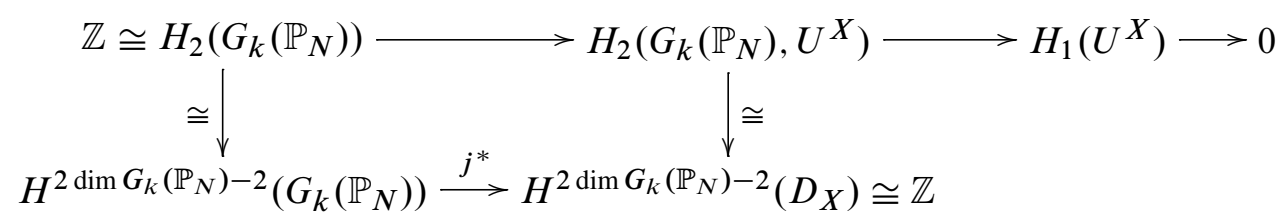

The vertical isomorphisms are Poincaré duality. Note that $H^{2 \operatorname{dim} G_{k}\left(\mathbb{P}_{N}\right)-2}\left(D_{X}\right) \cong \mathbb{Z}$ since $D_{X}$ is irreducible. The first horizontal sequence is exact and is a part of the homology sequence of the pair $\left(G_{k}\left(\mathbb{P}_{N}\right), U^{X}\right)$ and $j^{*}$ is induced by the inclusion $D_{X} \hookrightarrow G_{k}\left(\mathbb{P}_{N}\right)$. Then the generator of $H_{2}\left(G_{k}\left(\mathbb{P}_{N}\right)\right)$ is mapped to a positive integer times the generator of $H^{2 \operatorname{dim} G_{k}\left(\mathbb{P}_{N}\right)-2}\left(D_{X}\right)$, the fundamental class of $D_{X}$ (this positive integer is denoted by $\operatorname{deg} D_{X}$ and will be studied in the next section). Thus $H_{1}\left(U^{X}\right)$, which is isomorphic to the abelianization of $\pi_{1}\left(U^{X}\right)$, is a cyclic group of finite order. This completes the proof.

Now Theorem 3.1.1 is established. 


\subsection{Theory of Lefschetz pencils}

In this section we recall the definition of the degree of an analytic subset in a Grassmannian and describe a method to compute the degree of $D_{X} \subset G_{k}\left(\mathbb{P}_{N}\right)$. This will be used to compute the value of $\phi_{X}$ on a lasso.

First we treat the case of classical dual varieties, namely when $n=2$. Let $X \subset \mathbb{P}_{N}$ be a smooth projective surface. Then $G_{k}\left(\mathbb{P}_{N}\right)=G_{N-1}\left(\mathbb{P}_{N}\right)$ is nothing but the dual projective space $\mathbb{P}_{N}^{\vee}$ and $D_{X}$ is the dual variety of $X$. Let $L$ be a line of $\mathbb{P}_{N}^{\vee}$ avoiding the singular points of $D_{X}$ and meeting $D_{X}$ transversally. Note that generic lines of $\mathbb{P}_{N}^{\vee}$ satisfy this condition. We denote by $i_{L}$ the inclusion $L \hookrightarrow \mathbb{P}_{N}^{\vee}$. Then as explained by Katz [15] or Lamotke [17, (1.6.3)], $i_{L}^{*} \mathcal{W} \rightarrow L$ is a holomorphic Lefschetz fibration with the set of critical values being $L \cap D_{X}$, in the following sense.

Definition 3.4.1 Let $Y$ be a complex surface and $C$ a compact Riemann surface. A proper surjective holomorphic map $f: Y \rightarrow C$ is called a holomorphic Lefschetz fibration if the number of critical values of $f$ is finite and over each critical value, there exists only one critical point near which $f$ locally looks like $\left(z_{1}, z_{2}\right) \mapsto z_{1}^{2}+z_{2}^{2}$.

In particular deg $D_{X}$, which is equal to the number $\sharp\left(L \cap D_{X}\right)$ by definition, is equal to the number of critical points of $i_{L}^{*} \mathcal{W} \rightarrow L$.

More generally for a projective variety $X \subset \mathbb{P}_{N}$, we can take a generic line $L$ of $\mathbb{P}_{N}^{\vee}$ and consider the family of hyperplane sections $\{H \cap X\}_{H \in L}$ of $X$, parametrized by $L$. This construction is called Lefschetz pencils and very useful to study the topology of $X$.

We slightly generalize the above construction to the case of general $n$. We shall start by giving the definition of the degree of an analytic subset in a Grassmannian following [9, Chapter 3, Section 2-A].

Recall that $G_{p, q}$ is the Grassmannian of $p$-planes of $\mathbb{C}^{q}$. By a line of $G_{p, q}$ is meant a curve embedded in $G_{p, q}$ which can be written as

$$
P_{N M}:=\left\{W \in G_{p, q}: N \subset W \subset M\right\}
$$

for some $(p-1)$-plane $N$ and $(p+1)$-plane $M$ satisfying $N \subset M$.

Let $Z$ be an analytic subset of $G_{p, q}$. Take a line $P_{M N}$ of $G_{p, q}$ such that

(1) $P_{M N} \cap S(Z)=\varnothing$, where $S(Z)$ denotes the set of singular points of $Z$,

(2) $P_{M N}$ and $Z$ meet transversally. 
Then the intersection $P_{M N} \cap Z$ consists of finitely many points. We define $\operatorname{deg} Z \in \mathbb{Z}$ by $\operatorname{deg} Z:=\sharp\left(P_{M N} \cap Z\right)$

where $P_{M N}$ satisfies the above two conditions. When $Z$ is a hypersurface this number is positive (see [10, page 64]), and has the following topological interpretation. Let $c_{1}([Z]) \in H^{2}\left(G_{p, q} ; \mathbb{Z}\right)$ be the first Chern class of the line bundle over $G_{p, q}$ determined by $Z$ and $\left[P_{N M}\right] \in H_{2}\left(G_{p, q} ; \mathbb{Z}\right)$ the homology class represented by the embedded 1-dimensional projective space $P_{N M}$. Note that $\left[P_{N M}\right]$ is a generator of $H_{2}\left(G_{p, q} ; \mathbb{Z}\right) \cong \mathbb{Z}$. Then $\operatorname{deg} Z$ is equal to the result of the Kronecker pairing $\left\langle c_{1}([Z]),\left[P_{N M}\right]\right\rangle$. If $n=2$, this degree coincides with the usual definition of the degree of a projective hypersurface in $G_{N-1}\left(\mathbb{P}_{N}\right)=\mathbb{P}_{N}^{\vee}$. When the codimension of $Z$ is $\geq 2, \operatorname{deg} Z=0$.

We remark that generic lines of $G_{p, q}$ satisfy the above two conditions in the following sense. Let us consider the space parametrizing all lines of $G_{p, q}$; namely, let

$$
\mathcal{L}_{p, q}:=\left\{(N, M) \in G_{p-1, q} \times G_{p+1, q}: N \subset M\right\} .
$$

Then the set of $(N, M) \in \mathcal{L}_{p, q}$ such that $P_{N M}$ satisfying the above two conditions is nonempty and Zariski open in $\mathcal{L}_{p, q}$. This is proved by an application of Sard's lemma for varieties to the second projection $\left\{(z,(N, M)) \in Z \times \mathcal{L}_{p, q}: z \in P_{N M}\right\} \rightarrow \mathcal{L}_{p, q}$.

Let us return to our setting: $X \subset \mathbb{P}_{N}$ is a $n$-dimensional smooth projective variety, $D_{X}$ is the $k$-th associated variety. Let $E_{X}=E \subset D_{X}$ be as in Lemma 2.3.1 when the codimension of $D_{X}$ is $1, E_{X}=\varnothing$ when the codimension of $D_{X}$ is $\geq 2$. Here we put the subscript $X$ to $E$ to indicate its dependence on $X$. Let

$$
\mathcal{L}_{k}\left(\mathbb{P}_{N}\right):=\left\{(N, M) \in G_{k-1}\left(\mathbb{P}_{N}\right) \times G_{k+1}\left(\mathbb{P}_{N}\right): N \subset M\right\} .
$$

This is clearly isomorphic to $\mathcal{L}_{k+1, N+1}$. For $(N, M) \in \mathcal{L}_{k}\left(\mathbb{P}_{N}\right)$, the corresponding line $P_{N M} \subset G_{k}\left(\mathbb{P}_{N}\right)$ is defined by the same way as (3.4.2).

The existence of Lefschetz pencils for the case of general $n$ is stated as follows.

Theorem 3.4.3 (Existence of Lefschetz pencils) Let $P_{N M}$ be a line of $G_{k}\left(\mathbb{P}_{N}\right)$ not meeting $E_{X}$ and meeting $D_{X}$ transversally. Then the projection $i_{N M}^{*} \mathcal{W} \rightarrow P_{N M}$, where $i_{N M}: P_{N M} \hookrightarrow G_{k}\left(\mathbb{P}_{N}\right)$ denotes the inclusion, is a holomorphic Lefschetz fibration in the sense of Definition 3.4.1. Moreover $\operatorname{deg} D_{X}$ is equal to the number of critical points of $i_{N M}^{*} \mathcal{W} \rightarrow P_{N M}$.

By Theorem 2.3.12, the remaining to show is the number of critical points over each critical value is just one. If $n=2$, there is nothing to prove as remarked before Definition 3.4.1. We only remark that in the proof of (1.6.3) of [17], the biduality theorem plays a key role. 
To reduce the case of general $n$ to the case of $n=2$, we will cut $X$ with a generic $(k+1)$-plane. The result will be a smooth projective surface in the $(k+1)$-plane. We prepare some notation. $P_{N M}$ can be considered as a line of $M^{\vee}$, the dual projective space of $M$. Then we write it by $L_{N}$. For $M \in G_{k}\left(\mathbb{P}_{N}\right)$, let $X^{\prime}:=M \cap X \subset M$. If $M$ meets $X$ transversally, $X^{\prime}$ is a smooth surface in $M$. Then we can consider $D_{X^{\prime}}$ and $E_{X^{\prime}}$ in $M^{\vee}$.

Lemma 3.4.4 There exists a point $(N, M) \in \mathcal{L}_{k}\left(\mathbb{P}_{N}\right)$ such that

(1) the $(k+1)$-plane $M$ meets $X$ transversally (hence $X^{\prime}$ is a smooth projective surface),

(2) the line $P_{N M}$ does not meet $E_{X}$ and meets $D_{X}$ transversally,

(3) the line $L_{N}$ does not meet $E_{X^{\prime}}$ and meets $D_{X^{\prime}}$ transversally.

Proof The set of points in $\mathcal{L}_{k}\left(\mathbb{P}_{N}\right)$ satisfying the conditions 1 and 2 is nonempty and Zariski open in $\mathcal{L}_{k}\left(\mathbb{P}_{N}\right)$. Let $\left(N^{\prime}, M\right)$ be a point in this set. Since the set of lines of $M^{\vee}$ not meeting $E_{X^{\prime}}$ and meeting $D_{X^{\prime}}$ transversally is nonempty and Zariski open in the space of all lines of $M^{\vee}$, there exists a line $L_{N}$ near $L_{N^{\prime}}$ such that $(N, M)$ satisfies all the three conditions.

Implications of the Lemma Let $(N, M)$ be as in Lemma 3.4.4. We have the natural inclusion $\iota_{M}: M^{\vee} \hookrightarrow G_{k}\left(\mathbb{P}_{N}\right)$. Since $M$ meets $X$ transversally, for $H \in M^{\vee}$ the conditions $H \in D_{X^{\prime}}$ and $\iota_{M}(H) \in D_{X}$ are equivalent. Therefore we have the injection

$$
\left.\iota_{M}\right|_{U^{X^{\prime}}}: U^{X^{\prime}} \hookrightarrow U^{X},
$$

where $U^{X^{\prime}}=M^{\vee} \backslash D^{X^{\prime}}$, and the bijection $\left.\iota_{M}\right|_{L_{N} \cap D_{X^{\prime}}}: L_{N} \cap D_{X^{\prime}} \cong \rightarrow P_{N M} \cap D_{X}$. In particular, $D_{X}$ is a hypersurface if and only if $D_{X^{\prime}}$ is a hypersurface and we have $\operatorname{deg} D_{X}=\operatorname{deg} D_{X^{\prime}}$.

For simplicity we identify $L_{N} \cap D_{X^{\prime}}$ with $P_{N M} \cap D_{X}$ and write it by $D_{N M}$. Let $U_{N M}=P_{N M} \backslash D_{N M}$. By the inclusion $U_{N M} \hookrightarrow U^{X}$ (resp. $U_{N M} \hookrightarrow U^{X^{\prime}}$ ), any loop in $U_{N M}$ going once around a point of $D_{N M}$ is mapped to a lasso around $D_{X}$ (resp. $D_{X^{\prime}}$ ), hence a lasso around $D_{X^{\prime}}$ is mapped to a lasso around $D_{X}$ by the map (3.4.5). Consider the group homomorphism $j_{M}: \pi_{1}\left(U^{X^{\prime}}\right) \rightarrow \pi_{1}\left(U^{X}\right)$ induced by (3.4.5). The uniqueness of $\phi_{X^{\prime}}$ shows that $j_{M}^{*} \phi_{X}$ coincides with $\phi_{X^{\prime}}$. Thus the value of $\phi_{X}$ on a lasso around $D_{X}$ coincides with the value of $\phi_{X^{\prime}}$ on a lasso around $D_{X^{\prime}}$. In this way we can reduce the computation of $\operatorname{deg} D_{X}$ or the value of $\phi_{X}$ on a lasso around $D_{X}$ to the case of $n=2$.

Proof of Theorem 3.4.3 Let $(N, M)$ be as in Lemma 3.4.4. Let $i_{N}^{\prime}: L_{N} \rightarrow M^{\vee}$ be the inclusion and $\mathcal{W}^{\prime}:=\left\{(x, W) \in M \times M^{\vee}: x \in X^{\prime} \cap W\right\}$. We can consider the 
pullback $\left(i_{N}^{\prime}\right)^{*} \mathcal{W}^{\prime}$. Since $\operatorname{dim} X^{\prime}=2$ the remark right after the statement of Theorem 3.4.3 applies, so $\left(i_{N}^{\prime}\right)^{*} \mathcal{W}^{\prime} \rightarrow L_{N}$ is a holomorphic Lefschetz fibration. Therefore, $i_{N M}^{*} \mathcal{W} \rightarrow P_{N M}$ is also a holomorphic Lefschetz fibration because $\iota_{M}$ induces the isomorphism

$$
\left(i_{N}^{\prime}\right)^{*} \mathcal{W}^{\prime} \stackrel{\cong}{\longrightarrow} i_{N M}^{*} \mathcal{W}
$$

between the families of algebraic curves over $L_{N}=P_{N M}$. Thus we have proved that there exists a line $P_{0}=P_{N M}$ of $G_{k}\left(\mathbb{P}_{N}\right)$ not meeting $E_{X}$ and meeting $D_{X}$ transversally such that the projection $i_{N M}^{*} \mathcal{W} \rightarrow P_{N M}$ is a holomorphic Lefschetz fibration.

Let $P_{1}$ be a line not meeting $E_{X}$ and meeting $D_{X}$ transversally. For $j=0,1$, we denote the inclusion map $P_{j} \hookrightarrow G_{k}\left(\mathbb{P}_{N}\right)$ by $i_{j}$. Let $\mathcal{V}$ be the space of lines of $G_{k}\left(\mathbb{P}_{N}\right)$ not meeting $E_{X}$ and meeting $D_{X}$ transversally. This is nonempty and Zariski open, hence connected. Thus there exists a differentiable path in $\mathcal{V}$ joining $P_{0}$ and $P_{1}$, inducing a deformation equivalence of class $C^{\infty}$ between $i_{0}^{*} \mathcal{W}$ and $i_{1}^{*} \mathcal{W}$ as a family of algebraic curves over 1-dimensional projective space. We already know $i_{0}^{*} \mathcal{W} \rightarrow P_{0}$ is a holomorphic Lefschetz fibration, so $i_{1}^{*} \mathcal{W} \rightarrow P_{1}$ is also a holomorphic Lefschetz fibration.

Taking into account that $D_{X} \backslash E_{X}$ is connected, similar argument shows the following:

Corollary 3.4.6 In Theorem 2.3.12, the number of critical points of $f_{\imath}: \iota^{*} \mathcal{W} \rightarrow \Delta$ is 1. The singular fiber $f_{l}^{-1}(0)$ has just one nodal singularity and its topological type does not depend on the choice of $\iota$.

\subsection{Computations}

In this section we will give a formula for the value of $\phi_{X}$ on a lasso around $D_{X}$ from the data of various invariants of $X$. In view of the discussion of the implications of Lemma 3.4.4 in Section 3.4, we may focus on the case $n=2$.

First we review theory of Lefschetz pencils following [17]. Let $X \subset \mathbb{P}_{N}$ be a smooth projective surface and $L$ a generic line of $\mathbb{P}_{N}^{V}$, as in the beginning of Section 3.4. Let $D_{L}=L \cap D_{X}, U_{L}=L \backslash D_{L}$ and $X_{H}=H \cap X$ for $H \in L$. We have a family of Riemann surfaces over $U_{L}$ by restricting $i_{L}^{*} \mathcal{W} \rightarrow L$. Choosing a base point $H_{0} \in U_{L}$, let $\rho_{X}$ be the associated topological monodromy. The source of $\rho_{X}$ is the fundamental group $\pi_{1}\left(U_{L}\right)=\pi_{1}\left(U_{L}, H_{0}\right)$.

Picard-Lefschetz formula For $H^{\prime} \in D_{L}$ choose a path $\ell$ from $H_{0}$ to $H^{\prime}$ and let $\sigma$ be the element of $\pi_{1}\left(U_{L}\right)$ represented by a loop going to a point nearby $H^{\prime}$ along $\ell$, then going once around $H^{\prime}$ by counterclockwise manner and then coming back along $\ell$. 
Then the famous Picard-Lefschetz formula says that $\rho_{X}(\sigma)$ is the inverse (recall our conventions about monodromies) of the right hand Dehn twist along some simple closed curve $C_{\sigma}$, called the vanishing cycle, on $X_{H_{0}}$. The adjective "vanishing" comes in because looking at the fiber $H \cap X$ when $H$ moves along $\ell, X_{H^{\prime}}$ looks like obtained from $X_{H_{0}}$ by pinching $C_{\sigma}$ into a point.

Let $V$ be the submodule of $H_{1}\left(X_{H_{0}}\right)$ generated by all the vanishing cycles. Homology with coefficients in some principal ideal domain is considered. Then the equality

$$
V=\operatorname{Ker}\left(i_{*}: H_{1}\left(X_{H_{0}}\right) \rightarrow H_{1}(X)\right),
$$

where $i_{*}$ is induced by the inclusion $X_{H_{0}} \hookrightarrow X$, holds. See [17, (3.8.2)]. In particular, if $H_{1}(X)=0$, the vanishing cycles generate the homology of the reference fiber $X_{H_{0}}$.

Lemma 3.5.2 Suppose the genus of $X_{H_{0}}$ is positive and for some principal ideal domain $R$, the rank of $H_{1}(X)=H_{1}(X ; R)$ is less than twice the genus of $X_{H_{0}}$. Then every singular fiber of $i_{L}^{*} \mathcal{W} \rightarrow L$, ie, the inverse image of a point of $D_{L}$, is irreducible.

Proof First remark that for any choice of $H^{\prime}$ and $\ell, \sigma \in \pi_{1}\left(U_{L}\right)$ is mapped to a lasso around $D_{X}$ by the homomorphism $\pi_{1}\left(U_{L}\right) \rightarrow \pi_{1}\left(U^{X}\right)$ induced by the inclusion. Thus for any two vanishing cycles the Dehn twists along them are conjugate to each other in the mapping class group of $X_{H_{0}}$.

Suppose there exists a reducible fiber. This means that there exists a vanishing cycle which is a separating simple closed curve. Then all the vanishing cycles are separating by the remark above. Since any separating simple closed curve is zero as a homology class, this implies $V=0$. But by the assumption and (3.5.1) we also have $V=H_{1}\left(X_{H_{0}}\right) \neq 0$, a contradiction. This completes the proof.

Proposition 3.5.3 Let $X \subset \mathbb{P}_{N}$ be a smooth projective surface and $g$ the genus of a generic hyperplane section $H \cap X, H \in U^{X}$. Assume that $g>0$ and the rank of $H_{1}(X ; R)$ is less than $2 g$ for some principal ideal domain $R$. Suppose $D_{X}$ is a hypersurface and let $\sigma_{X} \in \pi_{1}\left(U^{X}\right)$ be a lasso around $D_{X}$. Then we have

$$
\phi_{X}\left(\sigma_{X}\right)=\frac{\operatorname{Sign} X-\operatorname{deg} X}{\chi(X)+\operatorname{deg} X-2(2-2 g)} .
$$

Here, Sign $X$ is the signature of $X$ as a closed oriented 4-manifold and $\chi(X)$ is the Euler-Poincaré characteristic of $X$, and $\operatorname{deg} X$ is the usual degree of $X$ (ie, the number of intersecting points with a generic complementary dimensional plane to $X$ ).

Proof Let $L$ be a generic line of $\mathbb{P}_{N}^{\vee}$ as in the beginning of this section. As in [17, (1.6.1)] the axis $A=\bigcap_{H \in L} H$ of the pencil meets $X$ transversally, and $i_{L}^{*} \mathcal{W}$ is the 
blow up of $X$ at the $\operatorname{deg} X$ points $A \cap X$ hence diffeomorphic to the connected sum $X \#(\operatorname{deg} X) \overline{\mathbb{P}}_{2}$. Therefore we have

$$
\operatorname{Sign}\left(i_{L}^{*} \mathcal{W}\right)=\operatorname{Sign} X-\operatorname{deg} X
$$

and $\chi\left(i_{L}^{*} \mathcal{W}\right)=\chi(X)+\operatorname{deg} X$.

Let $D_{L}=\left\{H_{1}, \ldots, H_{d}\right\}$, where $d=\operatorname{deg} D_{X}$ and for $1 \leq i \leq d$, let $\sigma_{i} \in \pi_{1}\left(U_{L}\right)$ be the element obtained by substituting $H_{i}$ for $H^{\prime}$ in the definition of $\sigma$; see the beginning of this section. As elements of $\pi_{1}\left(U^{X}\right)$, all $\sigma_{i}$ are lassos around $D_{X}$.

Let $D_{i} \subset L$ be a small closed 2-disk around $H_{i}$. We write by $f_{L}$ the projection $i_{L}^{*} \mathcal{W} \rightarrow L$ and write $X_{i}=f_{L}^{-1}\left(D_{i}\right)$. Let $X_{0}=X \backslash \coprod_{i}$ Int $X_{i}$. By Lemma 3.5.2, $f_{L}^{-1}\left(H_{i}\right)$ is irreducible hence the signature of $X_{i}$ is zero. Using the Novikov additivity, we have $\operatorname{Sign}\left(i_{L}^{*} \mathcal{W}\right)=\operatorname{Sign} X_{0}$. By Meyer's signature formula [20, Satz 1] and $\rho_{X}^{*} \tau_{g}=$ $\delta \phi_{X}$, we have

$$
\operatorname{Sign}\left(i_{L}^{*} \mathcal{W}\right)=\operatorname{Sign} X_{0}=\sum_{i=1}^{d} \phi_{X}\left(\sigma_{i}\right)=d \phi_{X}\left(\sigma_{X}\right)
$$

On the other hand since all the singular fibers have one nodal singularity, there are $d$ singular fibers with Euler contribution +1 (see [5, (11.4) Proposition]), thus

$$
d=\chi\left(i_{L}^{*} \mathcal{W}\right)-2(2-2 g)=\chi(X)+\operatorname{deg} X-2(2-2 g) .
$$

The proposition follows from (3.5.4), (3.5.5) and (3.5.6).

Note that by (3.5.6) we can express $\operatorname{deg} D_{X}$ in terms of $\chi(X), \operatorname{deg} X$, and $g$. The genus $g$ is expressed as follows. For $H \in U^{X}$, let $C=H \cap X$. By the adjunction formula we have $c_{1}(C)=\left.c_{1}(X)\right|_{C}-h$, where $h$ is the hyperplane class, thus

$$
2-2 g=\chi(C)=\left\langle c_{1}(C),[C]\right\rangle=\left\langle c_{1}(X) h-h^{2},[X]\right\rangle=\left\langle c_{1}(X) h,[X]\right\rangle-\operatorname{deg} X .
$$

Example 3.5.7 Let $m \geq 1$ and $n_{1}, \ldots, n_{m} \geq 2$ be integers and let $X \subset \mathbb{P}_{m+2}$ be a smooth complete intersection of type $\left(n_{1}, \ldots, n_{m}\right)$. Namely $X$ is given as the zero set of some homogeneous polynomials $f_{1}, \ldots, f_{m}$ where $f_{i}$ is of degree $n_{i}$.

Proposition 3.5.8 Let $X$ be as above and assume that $\left(m, n_{1}, \ldots, n_{m}\right) \neq(1,2)$. Then $D_{X}$ is a hypersurface. Let $\sigma_{X} \in \pi_{1}\left(U^{X}\right)$ be a lasso around $D_{X}$. We have

$$
\phi_{X}\left(\sigma_{X}\right)=\frac{m-\sum_{i=1}^{m} n_{i}^{2}}{3\left(\frac{m^{2}+m}{2}+\sum_{i=1}^{m} n_{i}^{2}-(m+1) \sum_{i=1}^{m} n_{i}+\sum_{i<j} n_{i} n_{j}\right)} .
$$


Proof We have $\operatorname{deg} X=n_{1} \cdots n_{m}$ and using the adjunction formula we can compute

$$
\chi(X)=c_{2}(X)
$$

$$
=n_{1} \cdots n_{m}\left(\left(\begin{array}{c}
m+3 \\
2
\end{array}\right)+\sum_{i=1}^{m} n_{i}^{2}-(m+3) \sum_{i=1}^{m} n_{i}+\sum_{i<j} n_{i} n_{j}\right),
$$

(3.5.10)

$$
\text { Sign } X=\frac{n_{1} \cdots n_{m}}{3}\left(m+3-\sum_{i=1}^{m} n_{i}^{2}\right) .
$$

For $H \in U^{X}, C=H \cap X$ is a smooth complete intersection of type $\left(n_{1}, \ldots, n_{m}, 1\right)$. Using the adjunction formula we have

$$
2-2 g=\chi(C)=n_{1} \cdots n_{m}\left(m+2-\sum_{i=1}^{m} n_{i}\right),
$$

and following the argument in the proof of Proposition 3.5.3, deg $D_{X}$ is given by

$$
\operatorname{deg} D_{X}=n_{1} \cdots n_{m}\left(\frac{m^{2}+m}{2}+\sum_{i=1}^{m} n_{i}^{2}-(m+1) \sum_{i=1}^{m} n_{i}+\sum_{i<j} n_{i} n_{j}\right) .
$$

We claim that $\operatorname{deg} D_{X}$ is positive. If $m=1, \operatorname{deg} D_{X}=n_{1}\left(n_{1}-1\right)^{2}>0$. If $m \geq 2$, Using the inequality

$$
\sum_{i=1}^{m} n_{i}^{2} \geq \frac{2}{m-1} \sum_{i<j} n_{i} n_{j}
$$

for $n_{i} \geq 0$ (this is easily derived from the geometric-arithmetic mean inequality), we have

$$
\begin{aligned}
\operatorname{deg} D_{X} & \geq n_{1} \cdots n_{m}\left(\frac{m^{2}+m}{2}+\frac{m+1}{m-1} \sum_{i<j} n_{i} n_{j}-(m+1) \sum_{i=1}^{m} n_{i}\right) \\
& =n_{1} \cdots n_{m} \frac{m+1}{m-1} \sum_{i<j}\left(n_{i}-1\right)\left(n_{j}-1\right) .
\end{aligned}
$$

Thus in any case deg $D_{X}>0$, ie, $D_{X}$ is a hypersurface. Also we can show $\chi(C) \leq 0$ hence $g>0$ except for the case $m=1$ and $n_{1}=2$. Finally, $X$ is simply connected. This follows from the Zariski theorem of Lefschetz type; see [17, (8.1.1)]. Now Proposition 3.5.3 can be applied, and combining the above computations all together we have the result. 
The next example is a generalization of the above, but it will illustrate that for a fixed variety, how the value of the Meyer function on a lasso depends on a choice of its projective embedding.

Example 3.5.12 Let $m \geq 0, n_{1}, \ldots, n_{m} \geq 2, n \geq 2$, and $d \geq 1$ be integers. When $m=0$, we assume that $d \geq 2$. Let $v_{d}: \mathbb{P}_{m+n} \hookrightarrow \mathbb{P}_{N}$ be the Veronese embedding of degree $d$. Here,

$$
N=\left(\begin{array}{c}
n+m+d \\
d
\end{array}\right)-1
$$

Let $X$ be the $v_{d}$-image of a smooth complete intersection in $\mathbb{P}_{m+n}$ of type $\left(n_{1}, \ldots, n_{m}\right)$. When $m=0, X$ is by definition the $v_{d}$-image of $\mathbb{P}_{n}$.

Proposition 3.5.13 Let $X$ be as above and assume that $\left(d, m, n_{1}, \ldots, n_{m}\right) \neq(1,1,2)$ and $(n, d, m) \neq(2,2,0)$. Then the $k$-th associated variety $D_{X}$ is a hypersurface. Let $\sigma_{X} \in \pi_{1}\left(U^{X}\right)$ be a lasso around $D_{X}$. We have

$$
\phi_{X}\left(\sigma_{X}\right)=\frac{\alpha_{X}}{\beta_{X}},
$$

where $\alpha_{X}=\frac{m+n+1-\sum_{i=1}^{m} n_{i}^{2}-(n+1) d^{2}}{3}$

and $\quad \beta_{X}=\left(\begin{array}{c}m+n+1 \\ 2\end{array}\right)+\sum_{i=1}^{m} n_{i}^{2}+\sum_{i<j} n_{i} n_{j}-(m+n+1)\left(\sum_{i=1}^{m} n_{i}+n d\right)$

$$
+n d \sum_{i=1}^{m} n_{i}+\frac{\left(n^{2}+n\right) d^{2}}{2} \text {. }
$$

Proof Let $(N, M) \in \mathcal{L}_{k}\left(\mathbb{P}_{N}\right)$ be as in Lemma 3.4.4 and $X^{\prime}=M \cap X$. We may focus on $X^{\prime} \subset M$. We will show that $D_{X^{\prime}}$ is a hypersurface and compute the value $\phi_{X^{\prime}}\left(\sigma_{X^{\prime}}\right)$, which must coincide with $\phi_{X}\left(\sigma_{X}\right)$, where $\sigma_{X^{\prime}} \in \pi_{1}\left(U^{X^{\prime}}\right)$ is a lasso around $D_{X^{\prime}}$.

First of all, the pullback $v_{d}^{-1}\left(X^{\prime}\right)$ is a smooth complete intersection in $\mathbb{P}_{m+n}$ of type

$$
(n_{1}, \ldots, n_{m}, \underbrace{d, \ldots, d}_{n-2}) .
$$

Thus $X^{\prime}$ is simply connected, and the invariants $\chi\left(X^{\prime}\right)$ and Sign $X^{\prime}$ can be computed from (3.5.9), (3.5.10). Also, we have $\operatorname{deg} X^{\prime}=\operatorname{deg} X=n_{1} \cdots n_{m} d^{n}$. From these we can see that $\operatorname{Sign} X^{\prime}-\operatorname{deg} X^{\prime}=n_{1} \cdots n_{m} d^{n-2} \alpha_{X}$. For $W \in U^{X^{\prime}} \subset U^{X}, C:=$ $W \cap X=W \cap X^{\prime}$ is a smooth complete intersection in $\mathbb{P}_{m+n}$ of type

$$
(n_{1}, \ldots, n_{m}, \underbrace{d, \ldots, d}_{n-1}) .
$$


Thus the genus $g$ of $C$ is seen by

$$
2-2 g=\chi(C)=n_{1} \cdots n_{m} d^{n-1}\left(m+n+1-\sum_{i=1}^{m} n_{i}-(n-1) d\right) .
$$

It is easy to see that under the assumption, we have $\chi(C) \leq 0$ hence $g>0$. Using (3.5.6) and our knowledge of $\chi\left(X^{\prime}\right), \operatorname{deg} X^{\prime}$, and $\chi(C)$ gives

$$
\operatorname{deg} D_{X^{\prime}}=n_{1} \cdots n_{m} d^{n-2} \beta_{X} .
$$

We claim that $\operatorname{deg} D_{X^{\prime}}$ is positive. Now we have the inequality

$$
n d^{2}+\sum_{i=1}^{m} n_{i}^{2} \geq \frac{2}{m+n-1}\left(\sum_{i<j} n_{i} n_{j}+n d \sum_{i=1}^{m} n_{i}+\left(\begin{array}{l}
n \\
2
\end{array}\right) d^{2}\right),
$$

the same kind as (3.5.11). Using this, we have

$$
\begin{array}{r}
\operatorname{deg} D_{X^{\prime}} \geq n_{1} \cdots n_{m} d^{n-2} \frac{m+n+1}{m+n-1}\left(\sum_{i<j}\left(n_{i}-1\right)\left(n_{j}-1\right)+n \sum_{i=1}^{m}\left(n_{i}-1\right)(d-1)\right. \\
\left.+\left(\begin{array}{l}
n \\
2
\end{array}\right)(d-1)^{2}\right) .
\end{array}
$$

This shows $\operatorname{deg} D_{X^{\prime}}>0$ except for the case $d=1$ and $m=1$. In this case, we have deg $D_{X^{\prime}}=n_{1}\left(n_{1}-1\right)^{2}>0$. Thus $D_{X^{\prime}}$ is a hypersurface, so is $D_{X}$. Applying Proposition 3.5.3, we have $\phi_{X}\left(\sigma_{X}\right)=\phi_{X^{\prime}}\left(\sigma_{X^{\prime}}\right)=\left(\operatorname{Sign} X^{\prime}-\operatorname{deg} X^{\prime}\right) / \operatorname{deg} D_{X^{\prime}}=$ $\alpha_{X} / \beta_{X}$, as desired.

\subsection{Bounded cohomology of $\pi_{1}\left(U^{X}\right)$}

For a group $G$, we denote by $H_{b}^{*}(G ; \mathbb{R})$ the bounded cohomology group of $G$. Namely, $H_{b}^{*}(G ; \mathbb{R})$ is the cohomology of the cochain complex of $\mathbb{R}$-valued bounded cochains of $G$. In this section we show that the second bounded cohomology of $\pi_{1}\left(U^{X}\right)$ is nontrivial under a certain mild condition.

Proposition 3.6.1 Let $X \subset \mathbb{P}_{N}$ be a smooth projective variety of dimension $\geq 2$ such that $D_{X}$ is a hypersurface. Suppose the value of $\phi_{X}$ on a lasso around $D_{X}$ is neither equal to 0 nor -1 . Then the bounded cohomology $H_{b}^{2}\left(\pi_{1}\left(U^{X}\right) ; \mathbb{R}\right)$ is nontrivial and the natural comparison map $H_{b}^{2}\left(\pi_{1}\left(U^{X}\right) ; \mathbb{R}\right) \rightarrow H^{2}\left(\pi_{1}\left(U^{X}\right) ; \mathbb{R}\right)$ is not injective. 
We need a lemma.

Lemma 3.6.2 Let $T \in \Gamma_{g}$ be the right hand Dehn twist along a nonseparating simple closed curve on $\Sigma_{g}$. Then for any integer $n \geq 1$, we have

$$
\tau_{g}\left(T^{-1}, T^{-n}\right)=-1
$$

Proof We use the description (1.0.4) of $\tau_{g}$. By the formulas (12) and (13) of [20], it suffices to prove $\operatorname{Sign}\left(V_{A, A^{n}},\langle,\rangle_{A, A^{n}}\right)=-1$ where

$$
A=\left(\begin{array}{cc}
1 & -1 \\
0 & 1
\end{array}\right)
$$

corresponding to the inverse of the right Dehn twist along a nonseparating simple closed curve on the torus. We have

$$
V_{A, A^{n}}=\left\{(x, y) \in \mathbb{R}^{2} \oplus \mathbb{R}^{2}:\left(\begin{array}{ll}
0 & 1 \\
0 & 0
\end{array}\right) x+\left(\begin{array}{cc}
0 & -n \\
0 & 0
\end{array}\right) y=0\right\},
$$

thus the vectors $\left((1,0)^{t},(0,0)^{t}\right),\left((0,0)^{t},(1,0)^{t}\right)$, and $\left((0, n)^{t},(0,1)^{t}\right)$ form a basis for $V_{A, A^{n}}$. The presentation matrix of $\langle,\rangle_{A, A^{n}}$ with respect to this basis is

$$
\left(\begin{array}{ccc}
0 & 0 & 0 \\
0 & 0 & 0 \\
0 & 0 & -n(n+1)
\end{array}\right) .
$$

This completes the proof.

Proof of Proposition 3.6.1 Note that $\tau_{g}$ is a bounded 2-cocycle of $\Gamma_{g}$. More precisely, for $f_{1}, f_{2} \in \Gamma_{g}$ we have $\left|\tau_{g}\left(f_{1}, f_{2}\right)\right| \leq 4 g$. Thus $\rho_{X}^{*} \tau_{g}$ is also a bounded 2 -cocycle. Since $\phi_{X}$ is a unique 1 -cochain cobounding $\rho_{X}^{*} \tau_{g}$, it suffices to show that $\phi_{X}$ is unbounded.

Let $\sigma_{X}$ be a lasso around $D_{X}$. By the Picard-Lefschetz formula, $\rho_{X}\left(\sigma_{X}\right)$ is the inverse of the right hand Dehn twist along a simple closed curve. We claim that this curve is nonseparating. For, if this is separating, $\rho_{X}\left(\sigma_{X}\right) \in \Gamma_{g}$ does act trivially on the homology of $\Sigma_{g}$. Combining this with the fact that $\pi_{1}\left(U^{X}\right)$ is normally generated by $\sigma_{X}$, we deduce that the image $\rho_{X}\left(\pi_{1}\left(U^{X}\right)\right)$ acts trivially on the homology of $\Sigma_{g}$. Hence $\rho_{X}^{*} \tau_{g}$ is zero as a cocycle. Since $\delta \phi_{X}=\rho_{X}^{*} \tau_{g}=0$ and $\phi_{X}\left(\sigma_{X}\right) \neq 0$ it follows that $\phi_{X}$ is a nontrivial homomorphism from $\pi_{1}\left(U^{X}\right)$ to $\mathbb{Q}$, contradicting to Lemma 3.3.1. 
Now by $\delta \phi_{X}=\rho_{X}^{*} \tau_{g}$ and Lemma 3.6.2, we have

$$
\phi_{X}\left(\sigma_{X}^{n}\right)=n \phi_{X}\left(\sigma_{X}\right)-\sum_{i=1}^{n-1} \tau_{g}\left(\rho_{X}\left(\sigma_{X}\right), \rho_{X}\left(\sigma_{X}^{i}\right)\right)=n \phi_{X}\left(\sigma_{X}\right)+n-1
$$

for $n \geq 1$. Since $\phi_{X}\left(\sigma_{X}\right) \neq-1$, this shows the unboundedness of $\phi_{X}$.

It is known that if a discrete group is amenable, then its bounded cohomology vanishes in positive degrees (see Gromov [11]). Thus:

Corollary 3.6.3 Let $X \subset \mathbb{P}_{N}$ be a smooth projective variety satisfying the hypothesis of Proposition 3.6.1. Then the fundamental group $\pi_{1}\left(U^{X}\right)$ is not amenable.

As an example, when $X$ is the one of those in Proposition 3.5.13, we can check that $\alpha_{X}<0$ and $\alpha_{X}+\beta_{X}>0$. Therefore Proposition 3.6.1 can be applied to this situation.

\section{Applications to local signatures}

\subsection{An approach to local signatures via Meyer functions}

Let $\mathbb{M}_{g}$ be the moduli space of compact Riemann surfaces of genus $g$ and $\mathcal{A}$ a subset of $\mathbb{M}_{g}$. We introduce the notion of an $\mathcal{A}$-fibration and a local signature with respect to $\mathcal{A}$.

Definition 4.1.1 Let $B$ be a topological space.

(1) A triple $\xi=(\mathcal{C}, p, B)$ is called an $\mathcal{A}$-family (on $B$ ) if $p: \mathcal{C} \rightarrow B$ is a continuous family of compact Riemann surfaces with each fiber being an element of $\mathcal{A}$.

(2) Let $\xi_{0}, \xi_{1}$ be $\mathcal{A}$-families on $B$. They are called isotopic if there exists an $\mathcal{A}$-family $\xi$ on $B \times[0,1]$ such that for $i=0,1$, the restriction of $\xi$ to $B \times\{i\}$ is isomorphic to $\xi_{i}$ as continuous family of Riemann surfaces on $B$, where $B \times\{i\}$ is identified with $B$ by $(b, i) \mapsto b$.

(3) We denote by $\mathcal{A}(B)$ the set of isotopy classes of $\mathcal{A}$-families over $B$.

Let $\xi=(\mathcal{C}, p, B)$ be an $\mathcal{A}$-family and $\psi: B^{\prime} \rightarrow B$ a continuous map. By taking the fiber product of $\psi$ and $p$, the pullback $\psi^{*} \xi$ by $\psi$ is naturally defined as an $\mathcal{A}$-family on $B^{\prime}$. In this way we get a category of $\mathcal{A}$-families, which we denote by $\mathcal{A}^{\prime}$. Moreover this association induces the map $\eta_{\xi, B^{\prime}}:\left[B^{\prime}, B\right] \rightarrow \mathcal{A}\left(B^{\prime}\right)$, where $\left[B^{\prime}, B\right]$ is the set of homotopy classes of continuous maps from $B^{\prime}$ to $B$. 
Definition 4.1.2 An $\mathcal{A}$-family $\xi_{u}=\left(\mathcal{C}_{u}, p_{u}, B_{u}\right)$ over a path connected space $B_{u}$ having the homotopy type of a CW-complex is called universal if $\eta_{\xi_{u}, B}$ is bijective for any topological space $B$ having the homotopy type of a CW-complex. We denote by $\rho_{u}: \pi_{1}\left(B_{u}\right) \rightarrow \Gamma_{g}$ the topological monodromy of $p_{u}: \mathcal{C}_{u} \rightarrow B_{u}$.

A universal $\mathcal{A}$-family is uniquely determined up to isotopy if it exists: if $\xi_{u}^{\prime}=$ $\left(\mathcal{C}_{u}^{\prime}, p_{u}^{\prime}, B_{u}^{\prime}\right)$ is another universal $\mathcal{A}$-family then there exist continuous maps $\psi: B_{u} \rightarrow$ $B_{u}^{\prime}$ and $\psi^{\prime}: B_{u}^{\prime} \rightarrow B_{u}$ such that $\psi^{*} \xi_{u}^{\prime}$ and $\xi_{u}$ (resp. $\psi^{\prime *} \xi_{u}$ and $\xi_{u}^{\prime}$ ) are isotopic. In particular $B_{u}$ and $B_{u}^{\prime}$ are homotopy equivalent.

In some situations as we will see, we can construct a universal $\mathcal{A}$-family from a certain $\mathcal{A}$-family with group action. The following proposition is used to verify the universality of such a family. To state the proposition we prepare a terminology. Let $\xi^{0}=\left(\mathcal{C}^{0}, p^{0}, B^{0}\right)$ be a $\mathcal{A}$-family on a connected $C^{\infty}$-manifold $B^{0}$, and let $\mathcal{G}$ be a Lie group acting on $\mathcal{C}^{0}$ and $B^{0}$ from the left such that $p^{0}$ is $\mathcal{G}$-equivariant. Let $\mathcal{G}^{\prime}$ be the category defined as follows: the objects consist of $(P, \pi, B, E)$ such that $\pi: P \rightarrow B$ is a principal $\mathcal{G}$-bundle on $B$ (the $\mathcal{G}$-action on $P$ being from the left) and $E: P \rightarrow B^{0}$ is a $\mathcal{G}$-equivariant map, and the morphisms from $(P, \pi, B, E)$ and $\left(P^{\prime}, \pi^{\prime}, B^{\prime}, E^{\prime}\right)$ are the bundle maps from $P$ to $P^{\prime}$ compatible with $E$ and $E^{\prime}$.

Proposition 4.1.3 (A criterion for universality) Let $\mathcal{A}$ be a subset of $\mathbb{M}_{g}$ and $\xi^{0}=$ $\left(\mathcal{C}^{0}, p^{0}, B^{0}\right)$ an $\mathcal{A}$-family as above. Suppose there is a covariant functor from $\mathcal{A}^{\prime}$ to $\mathcal{G}^{\prime}$ associating an $\mathcal{A}$-family $\xi=(\mathcal{C}, p, B)$ with $\left(P(\xi), \pi, B, E_{\xi}\right)$, and satisfying the following conditions:

(1) $E_{\xi}^{*} \xi^{0}$ and $\pi^{*} \xi$ are isomorphic as continuous families of Riemann surfaces on $P(\xi)$.

(2) As for the object associated to $\xi^{0}$, we can take a trivial $\mathcal{G}$-bundle $P\left(\xi^{0}\right)=\mathcal{G} \times B^{0}$ and a $\mathcal{G}$-equivariant map $E_{\xi 0}$ such that $E_{\xi^{0}}(g, b)=g \cdot b$. Moreover, for any $g \in \mathcal{G}$ the bundle map $\bar{g}: P\left(\xi^{0}\right) \rightarrow P\left(\xi^{0}\right)$ induced by the maps $B^{0} \rightarrow B^{0}$, $b \mapsto g \cdot b$ and $\mathcal{C}^{0} \rightarrow \mathcal{C}^{0}, c \mapsto g \cdot c$ is given by $\bar{g}\left(g^{\prime}, b\right)=\left(g^{\prime} g^{-1}, g \cdot b\right)$.

Let $E \mathcal{G} \rightarrow B \mathcal{G}$ be a universal principal $\mathcal{G}$-bundle (the $\mathcal{G}$-action on $E \mathcal{G}$ being from the right). Taking the Borel constructions $B_{\mathcal{G}}^{0}=E \mathcal{G} \times_{\mathcal{G}} B^{0}$ and $\mathcal{C}_{\mathcal{G}}^{0}$ we obtain an $\mathcal{A}$-family $\xi_{\mathcal{G}}^{0}=\left(\mathcal{C}_{\mathcal{G}}^{0}, p_{\mathcal{G}}^{0}, B_{\mathcal{G}}^{0}\right)$. Then, $\xi_{\mathcal{G}}^{0}$ is a universal $\mathcal{A}$-family.

Proof Let $B$ be a space having the homotopy type of a CW-complex. For simplicity we write $\eta=\eta_{\xi_{\mathcal{G}}^{0}, B}$. We construct a candidate for the inverse of $\eta$. Let $\xi=(\mathcal{C}, p, B)$ be an $\mathcal{A}$-family on $B$. Take a principal $\mathcal{G}$-bundle $P=P(\xi)$ and a $\mathcal{G}$-equivariant map $E=E_{\xi}$ associated to $\xi$. Considering the Borel construction $P_{\mathcal{G}}=E \mathcal{G} \times \mathcal{G} P$, let 
$T: P_{\mathcal{G}} \rightarrow B$ be the map induced from the projection $\pi: P \rightarrow B$. This is an $E \mathcal{G}$-bundle, thus by Dold's theorem it has a section: a map $\zeta: B \rightarrow P_{\mathcal{G}}$ such that $T \circ \zeta=\operatorname{id}_{B}$. Let $E_{\mathcal{G}}: P_{\mathcal{G}} \rightarrow B_{\mathcal{G}}^{0}$ be the map induced from $E$. Now the isomorphism $E^{*} \xi^{0} \cong \pi^{*} \xi$ induces the isomorphism $E_{\mathcal{G}}{ }^{*} \xi_{\mathcal{G}}^{0} \cong T^{*} \xi$ and $E_{\mathcal{G}} \circ \zeta$ is a continuous map from $B$ to $B_{\mathcal{G}}^{0}$ such that

$$
\left(E_{\mathcal{G}} \circ \zeta\right)^{*} \xi_{\mathcal{G}}^{0}=\zeta^{*} E_{\mathcal{G}}{ }^{*} \xi_{\mathcal{G}}^{0} \cong \zeta^{*} T^{*} \xi=(T \circ \zeta)^{*} \xi=\xi
$$

This shows $\eta$ is surjective. In fact, using the functoriality we can show that the homotopy class of $E_{\mathcal{G}} \circ \zeta$ depends only on the isotopy class of $\xi$. In this way we have the map $\theta: \mathcal{A}(B) \rightarrow\left[B, B_{\mathcal{G}}^{0}\right]$ satisfying $\eta \circ \theta=i d_{\mathcal{A}(B)}$.

Here we consider the above construction applied to $\xi_{\mathcal{G}}^{0}$. We have $P\left(\xi^{0}\right)=\mathcal{G} \times B^{0}$ with the projection $\pi^{0}: P\left(\xi^{0}\right) \rightarrow B^{0},(g, b) \mapsto b$ and the $\mathcal{G}$-equivariant map $E_{\xi^{0}}: P\left(\xi^{0}\right) \rightarrow$ $B^{0},(g, b) \mapsto g \cdot b$. By the functoriality, the $\mathcal{G}$-bundle $\pi_{u}: P^{u} \rightarrow B_{\mathcal{G}}^{0}$ and the $\mathcal{G}-$ equivariant map $E^{u}: P^{u} \rightarrow B^{0}$ associated to $\xi_{\mathcal{G}}^{0}$ is described as follows.

Take the Borel construction $P^{u}:=E \mathcal{G} \times_{\mathcal{G}} P\left(\xi^{0}\right)$ where the $\mathcal{G}$-action on $P\left(\xi^{0}\right)$ is given by $g \cdot\left(g^{\prime}, b\right)=\left(g^{\prime} g^{-1}, g \cdot b\right)$. Define $\pi_{u}: P^{u} \rightarrow B_{\mathcal{G}}^{0}$ and $E^{u}: P^{u} \rightarrow B^{0}$ by

$$
\pi_{u}([e,(g, b)])=[e, b] \text {, and } E^{u}([e,(g, b)])=g \cdot b,
$$

where $[e, b]$ denotes the element of $B_{\mathcal{G}}^{0}$ represented by $(e, b) \in E \mathcal{G} \times B^{0}$, etc. Given the $\mathcal{G}$-action on $P^{u}$ by $g \cdot\left[e,\left(g^{\prime}, b\right)\right]=\left[e,\left(g g^{\prime}, b\right)\right], \pi_{u}$ is a principal $\mathcal{G}$-bundle and $E^{u}$ is $\mathcal{G}$-equivariant. Also the isomorphism $E_{\xi^{0}}{ }^{*} \xi_{0} \cong \pi^{0^{*}} \xi_{0}$ induces $E^{u *} \xi_{0} \cong \pi_{u}{ }^{*} \xi_{\mathcal{G}}^{0}$. Notice that $T_{u}: P_{\mathcal{G}}^{u} \rightarrow B_{\mathcal{G}}^{0}$ has a section $\zeta_{u}$ given by $\zeta_{u}([e, b])=\left[e,\left[e,\left(\operatorname{id}_{\mathcal{G}}, b\right)\right]\right]$.

Now we show $\theta \circ \eta=\operatorname{id}_{\left[B, B_{\mathcal{G}}^{0}\right]}$, which will complete the proof. Let $\psi: B \rightarrow B_{\mathcal{G}}^{0}$ be a continuous map. By the functoriality, we can use the fiber product $\psi^{*} P^{u}$ as the $\mathcal{G}$-bundle associated to $\psi^{*} \xi_{\mathcal{G}}^{0}$. Pulling back $\zeta_{u}$, we have a section $\psi^{*} \zeta_{u}$ of $T:\left(\psi^{*} P^{u}\right)_{\mathcal{G}} \rightarrow B$ which makes the following diagram commutative.

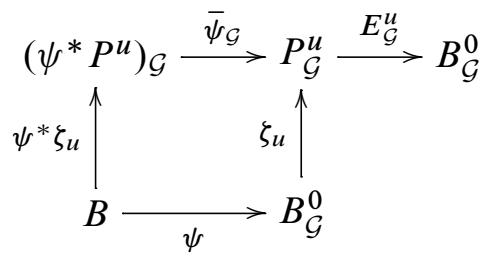

Notice that $E_{\mathcal{G}}^{u} \circ \zeta_{u}=\mathrm{id}_{B_{\mathcal{G}}^{0}}$. Following the construction of $\theta$ we have

$$
\theta\left(\left[\psi^{*} \xi_{\mathcal{G}}^{0}\right]\right)=\left[E_{\mathcal{G}}^{u} \circ \bar{\psi}_{\mathcal{G}} \circ \psi^{*} \zeta_{u}\right]=\left[E_{\mathcal{G}}^{u} \circ \zeta_{u} \circ \psi\right]=[\psi],
$$

which shows $\theta \circ \eta=\operatorname{id}_{\left[B, B_{\mathcal{G}}^{0}\right]}$. 
Definition 4.1.4 (1) Let $\Delta$ be a closed oriented 2-disk with the center $b$. A $4^{\circ}$ tuple $\mathcal{F}=(S, f, \Delta, b)$ is called an $\mathcal{A}$-degeneration if $S$ is a $C^{\infty}$-manifold of dimension 4 and $f: S \rightarrow \Delta$ is a proper surjective $C^{\infty}$-map, and the restriction of $f$ to $\Delta \backslash\{b\}$ is given a structure of $\mathcal{A}$-family. We denote by $\xi_{\mathcal{F}}$ this $\mathcal{A}$-family.

(2) Let $\mathcal{F}=(S, f, \Delta, b)$ and $\mathcal{F}^{\prime}=\left(S^{\prime}, f^{\prime}, \Delta^{\prime}, b^{\prime}\right)$ be $\mathcal{A}$-degenerations. We say $\mathcal{F}$ and $\mathcal{F}^{\prime}$ are equivalent if taking suitably smaller disks $\Delta_{0} \subset \Delta$ with $b \in \Delta_{0}$ and $\Delta_{0}^{\prime} \subset \Delta^{\prime}$ with $b^{\prime} \in \Delta_{0}^{\prime}$, there exist an orientation preserving homeomorphism $\psi:\left(\Delta_{0}, b\right) \rightarrow\left(\Delta_{0}^{\prime}, b^{\prime}\right)$ such that $\psi^{*} \xi_{\mathcal{F}^{\prime}}$ is isotopic to the restriction of $\xi_{\mathcal{F}}$ to $\Delta_{0} \backslash\{b\}$.

(3) We denote by $\mathcal{A}_{\text {loc }}$ the set of all equivalence classes of $\mathcal{A}$-degenerations. We often identify an element of $\mathcal{A}_{\text {loc }}$ with its representative. Each element of $\mathcal{A}_{\text {loc }}$ is called a fiber germ. A smooth fiber germ is an element of $\mathcal{A}_{\text {loc }}$ obtained by an $\mathcal{A}$-family $\xi=(\mathcal{C}, p, \Delta)$.

Definition 4.1.5 Let $M$ be a closed oriented 4-manifold and $B$ a closed oriented 2-manifold. A proper surjective $C^{\infty}$-map $f: M \rightarrow B$ is called an $\mathcal{A}$-fibration if there exist finitely many points $b_{1}, \ldots, b_{m} \in B$ such that the restriction of $f$ to $B \backslash\left\{b_{1}, \ldots, b_{m}\right\}$ is given a structure of $\mathcal{A}$-family.

The triple $(M, f, B)$ is a fibered 4-manifold in the sense of Section 1. For an $\mathcal{A}_{-}$ fibration $f: M \rightarrow B$, let $\mathcal{F}_{i}$ be the element of $\mathcal{A}_{\text {loc }}$ obtained by restricting $f$ to a small closed disk neighborhood $\Delta_{i}$ of $b_{i}$. We formulate the notion of a local signature in our setting.

Definition 4.1.6 Let $\mathcal{A}$ be a subset of $\mathbb{M}_{g}$. A function $\sigma_{\mathcal{A}}: \mathcal{A}_{\mathrm{loc}} \rightarrow \mathbb{Q}$ is called a local signature with respect to $\mathcal{A}$ if

(1) for a smooth fiber germ $\mathcal{F}, \sigma_{\mathcal{A}}(\mathcal{F})=0$, and

(2) for any $\mathcal{A}$-fibration $f: M \rightarrow B$, we have the global signature formula:

$$
\operatorname{Sign}(M)=\sum_{i=1}^{m} \sigma_{\mathcal{A}}\left(\mathcal{F}_{i}\right) .
$$

Proposition 4.1.8 Let $\mathcal{A}$ be a subset of $\mathbb{M}_{g}$. Suppose there exist a universal $\mathcal{A}$ family $\xi_{u}=\left(\mathcal{C}_{u}, p_{u}, B_{u}\right)$ and a $\mathbb{Q}$-valued 1 -cochain $\phi_{\mathcal{A}}: \pi_{1}\left(B_{u}\right) \rightarrow \mathbb{Q}$ such that $\delta \phi_{\mathcal{A}}=\rho_{u}^{*} \tau_{g}$. Then there exists a local signature with respect to $\mathcal{A}$.

Proof Let $\mathcal{F}=(S, f, \Delta, b) \in \mathcal{A}_{\mathrm{loc}}$. Since $\eta=\eta_{\xi_{u}, \Delta \backslash\{b\}}$ is bijective, there exists uniquely up to homotopy a continuous map $g_{\mathcal{F}}: \Delta \backslash\{b\} \rightarrow B_{u}$ such that $\eta\left(\left[g_{\mathcal{F}}\right]\right)=\left[\xi_{\mathcal{F}}\right]$. 
We denote by $\partial \Delta$ the element of $\pi_{1}(\Delta \backslash\{b\})$ represented by the loop going once around the boundary of $\Delta$ by counterclockwise manner. Then we obtain an element $x_{\mathcal{F}}=g_{\mathcal{F}_{*}}(\partial \Delta) \in \pi_{1}\left(B_{u}\right)$, which is uniquely determined up to conjugacy. Since the equality $\delta \phi_{\mathcal{A}}=\rho_{u}^{*} \tau_{g}$ implies that $\phi_{\mathcal{A}}$ is a class function (see Section 1), the value $\phi_{\mathcal{A}}\left(x_{\mathcal{F}}\right)$ is well defined.

Now define $\sigma_{\mathcal{A}}: \mathcal{A}_{\text {loc }} \rightarrow \mathbb{Q}$ by

$$
\sigma_{\mathcal{A}}(\mathcal{F})=\phi_{\mathcal{A}}\left(x_{\mathcal{F}}\right)+\operatorname{Sign}(S) .
$$

If $\mathcal{F}$ is a smooth fiber germ, $g_{\mathcal{F}}$ extends to a continuous map from $\Delta$. So $x_{\mathcal{F}} \in \pi_{1}\left(B_{u}\right)$ is trivial, hence $\phi_{\mathcal{A}}\left(x_{\mathcal{F}}\right)=0$. Also we have $\operatorname{Sign}(S)=0$ since topologically $f: S \rightarrow \Delta$ is just a trivial $\Sigma_{g}$-bundle. The first condition in Definition 4.1.6 is verified. The second condition is verified by an argument similar to the proof of Proposition 3.5.3, so we omit the detail (see also [16, Theorem 7.2]).

Definition 4.1.10 For $\mathcal{F} \in \mathcal{A}_{\text {loc }}$, we call $x_{\mathcal{F}} \in \pi_{1}\left(B_{u}\right)$ appeared in the proof of Proposition 4.1 .8 the lifted monodromy. This is uniquely determined up to conjugacy.

\subsection{Fibrations of rank 4 nonhyperelliptic curves of genus 4}

Let $C$ be a nonhyperelliptic Riemann surface of genus 4 . Its canonical image is a $(2,3)$ complete intersection in $\mathbb{P}_{3}$ hence is contained in a uniquely determined quadric. We say $C$ is of rank 4 if this quadric is of rank 4 . Let $\mathcal{R}^{4} \subset \mathbb{M}_{4}$ be the set of rank 4 nonhyperelliptic Riemann surfaces of genus $4 . \mathcal{R}^{4}$ is Zariski open in $\mathbb{M}_{4}$.

Let $s: \mathbb{P}_{1} \times \mathbb{P}_{1} \rightarrow \mathbb{P}_{3}$ be the Segre embedding. Explicitly, $s$ is given by

$$
s\left(\left[a_{0}: a_{1}\right],\left[b_{0}: b_{1}\right]\right)=\left[a_{0} b_{0}: a_{0} b_{1}: a_{1} b_{0}: a_{1} b_{1}\right],
$$

using the homogeneous coordinates. Let $V_{3,3}=\mathbb{C}\left[a_{0}, a_{1}\right]^{3} \otimes \mathbb{C}\left[b_{0}, b_{1}\right]^{3}$ be the space of $(3,3)$ homogeneous polynomials, and let

$$
s_{3,3}: \mathbb{P}_{1} \times \mathbb{P}_{1} \rightarrow \mathbb{P}\left(V_{3,3} \vee\right) \cong \mathbb{P}\left(V_{3,3}\right)^{\vee} \cong \mathbb{P}_{15}
$$

be the embedding induced from the evaluation map $\mathbb{C}^{2} \times \mathbb{C}^{2} \rightarrow V_{3,3}{ }^{\vee}=\operatorname{Hom}\left(V_{3,3}, \mathbb{C}\right)$. Set

$$
X=\operatorname{Im}\left(s_{3,3}\right) .
$$

Consider the group $\mathcal{G}=\operatorname{Aut}\left(\mathbb{P}_{1} \times \mathbb{P}_{1}\right)$. Of course $\mathcal{G}$ acts on $\mathbb{P}_{1} \times \mathbb{P}_{1}$ (from the left), inducing an action of $\mathcal{G}$ on $\mathbb{P}_{15}$ so that $s_{3,3}$ is $\mathcal{G}$-equivariant. Moreover $\mathcal{G}$ naturally acts on $\mathbb{P}\left(V_{3,3}\right)$ from the left.

Let $D_{X} \subset \mathbb{P}_{15}^{\vee}=\mathbb{P}\left(V_{3,3}\right)$ be the dual variety of $X$ and $U^{X}=\mathbb{P}\left(V_{3,3}\right) \backslash D_{X} . D_{X}$ is preserved by the $\mathcal{G}$-action. Also $\mathcal{G}$ acts on $\mathcal{C}^{X} \subset \mathbb{P}_{15} \times U^{X}$ diagonally, and the 
projection $p_{X}: \mathcal{C}^{X} \rightarrow U^{X}$ is $\mathcal{G}$-equivariant. Note that for $W \in U^{X}$, the fiber $p_{X}^{-1}(W)$ is isomorphic to the smooth curve in $\mathbb{P}_{1} \times \mathbb{P}_{1}$ determined by a $(3,3)$ homogeneous polynomial, which is an element of $\mathcal{R}^{4}$ since the restriction of $s$ to the curve gives its canonical embedding and the image is contained in $s\left(\mathbb{P}_{1} \times \mathbb{P}_{1}\right)$, which is a smooth quadric $x_{0} x_{3}-x_{1} x_{2}=0$. Thus, $\xi^{X}=\left(\mathcal{C}^{X}, p_{X}, U^{X}\right)$ is a $\mathcal{R}^{4}$-family.

Now we will show that $\xi^{X}$ and the $\mathcal{G}$-action on it satisfies the conditions in Proposition 4.1.3. We need to construct a principal $\mathcal{G}$-bundle from a $\mathcal{R}^{4}$-family.

First we consider the case of a single element $C \in \mathcal{R}^{4}$. We denote by $\Omega^{1}(C)$ the space of holomorphic 1 -forms on $C$. The unique quadric containing the canonical image of $C$ corresponds to the 1-dimensional kernel of the natural map $t_{2}: \operatorname{Sym}^{2} \Omega^{1}(C) \rightarrow$ $H^{0}\left(C ; K_{C}^{\otimes 2}\right)$. Here, $K_{C}$ is the canonical bundle of $C$. Note that $t_{2}$ is surjective by Max Noether's theorem (see [10, page 253]).

If we take a basis $\omega=\left(\omega_{0}, \omega_{1}, \omega_{2}, \omega_{3}\right)$ of $\Omega^{1}(C)$, an explicit form of $\operatorname{Ker}\left(t_{2}\right)$ is obtained as follows. Let $\varphi_{0}, \varphi_{1}, \varphi_{2}, \varphi_{3} \in \Omega^{1}(C)^{*}$ be the dual basis of $\omega$. Then Sym $^{2} \Omega^{1}(C)$ is identified with the space $S_{4}$ of $4 \times 4$ symmetric matrices by assigning $B \in S_{4}$ with the quadratic function

$$
\Omega^{1}(C)^{*} \rightarrow \mathbb{C}, x_{0} \varphi_{0}+x_{1} \varphi_{1}+x_{2} \varphi_{2}+x_{3} \varphi_{3} \mapsto\left(x_{0}, x_{1}, x_{2}, x_{3}\right) B\left(x_{0}, x_{1}, x_{2}, x_{3}\right)^{t} .
$$

Hence a choice of a basis $\omega$ of $\Omega^{1}(C)$ determines the element $B(\omega) \in \mathbb{P}\left(S_{4}\right)$ corresponding to $\operatorname{Ker}\left(t_{2}\right)$, and the image of the canonical map $\iota_{\omega}: C \hookrightarrow \mathbb{P}_{3}, c \mapsto\left[\omega_{0}(c)\right.$ : $\left.\omega_{1}(c): \omega_{2}(c): \omega_{3}(c)\right]$ is contained in the quadric determined by $B(\omega)$.

Let $P(C)$ be the set of $\omega$ modulo $\mathbb{C}^{*}$ such that the quadric determined by $B(\omega)$ is equal to $\left\{x_{0} x_{3}-x_{1} x_{2}=0\right\}$. Namely,

$$
P(C)=\left\{\omega \bmod \mathbb{C}^{*}: \omega \text { is a basis of } \Omega^{1}(C) \text { and } B(\omega)=H\right\},
$$

where $H \in \mathbb{P}\left(S_{4}\right)$ is represented by

$$
\left(\begin{array}{cccc}
0 & 0 & 0 & 1 \\
0 & 0 & -1 & 0 \\
0 & -1 & 0 & 0 \\
1 & 0 & 0 & 0
\end{array}\right) .
$$

Now consider the group $\mathrm{PO}_{4}^{H}(\mathbb{C})=\left\{A \in \operatorname{PGL}(4): A^{t} H A=H\right\}$, which acts on $P(C)$ from the left freely and transitively by $A \cdot\left(\omega_{0}, \omega_{1}, \omega_{2}, \omega_{3}\right) \bmod \mathbb{C}^{*}=$ $\left(\omega_{0}, \omega_{1}, \omega_{2}, \omega_{3}\right) A^{t} \bmod \mathbb{C}^{*}$. In fact, this group is isomorphic to $\mathcal{G}$ and the isomorphism is induced by the action of $\mathrm{PO}_{4}^{H}(\mathbb{C})$ on $\mathbb{P}_{3}$ (as a subgroup of PGL(4)) preserving $s\left(\mathbb{P}_{1} \times \mathbb{P}_{1}\right)$. Therefore, $\mathcal{G}$ acts on $P(C)$ freely and transitively. Finally, define the map

$$
E_{C}: P(C) \rightarrow U^{X}
$$


as follows. Again by Max Noether's theorem, the natural map $t_{3}: \operatorname{Sym}^{3} \Omega^{1}(C) \rightarrow$ $H^{0}\left(C ; K_{C}^{\otimes 3}\right)$ is surjective. Choose $h \in \operatorname{Ker}\left(t_{3}\right)$ which is not divided by elements of $\operatorname{Ker}\left(t_{2}\right)$. Let $\omega \in P(C)$. Then $h$ is identified with a homogeneous polynomial of degree 3 in determinates $x_{0}, x_{1}, x_{2}, x_{3}$. We denote it by $h^{\omega}$. The canonical image $\iota_{\omega}(C)$ is given by $x_{0} x_{3}-x_{1} x_{2}=h^{\omega}\left(x_{0}, x_{1}, x_{2}, x_{3}\right)=0$. Set

$$
E_{C}(\omega)=h^{\omega}\left(a_{0} b_{0}, a_{0} b_{1}, a_{1} b_{0}, a_{1} b_{1}\right) \in \mathbb{P}\left(V_{3,3}\right) .
$$

Since the zero set of $h^{\omega}\left(a_{0} b_{0}, a_{0} b_{1}, a_{1} b_{0}, a_{1} b_{1}\right)$ is isomorphic to $C$, we have $E_{C}(\omega) \in$ $U^{X} . E_{C}(\omega)$ does not depend on the choice of $h$, and we can verify that $E_{C}$ is $\mathcal{G}-$ equivariant.

Now let $\xi=(\mathcal{C}, p, B)$ be a $\mathcal{R}^{4}$-family. Applying the above construction to all the fibers, we get a principal $\mathcal{G}$-bundle

$$
P(\xi)=\bigcup_{b \in B} P\left(p^{-1}(b)\right)
$$

and by piecing together $E_{p^{-1}(b)}, b \in B$, we get a $\mathcal{G}$-equivariant map

$$
E_{\xi}: P(\xi) \rightarrow U^{X}
$$

The first condition in Proposition 4.1.3 is clear from the construction. So far we have only used the objects arising from holomorphic 1-forms on Riemann surfaces, which behave naturally under pullback by biholomorphic maps. Thus the functoriality is also true. As to the second condition, we can describe $P\left(\xi^{X}\right)$ as follows. For $W \in U^{X}$, $W \cap X$ is isomorphic to $v_{3}^{-1}(W \cap X) \subset \mathbb{P}_{3}$. Let $\omega_{W}$ be the basis of $\Omega^{1}(W \cap X)$ corresponding to the homogeneous coordinates $\left[x_{0}: x_{1}: x_{2}: x_{3}\right]$ of $\mathbb{P}_{3}$. Then the isomorphism

$$
P\left(\xi_{X}\right) \cong \mathcal{G} \times U^{X}
$$

is given by assigning $(A, W) \in \mathcal{G} \times U^{X}$ with $A \cdot \omega_{W}$. We can check that for $A \in \mathcal{G}$ and $W \in U^{X}, \omega_{W}$ corresponds to $A^{-1} \cdot \omega_{A \cdot W}$ by the isomorphism $W \cap X \rightarrow(A \cdot W) \cap X$ induced by $A$. The second condition follows from this. Applying Proposition 4.1.3, we have a universal $\mathcal{R}^{4}$-family $\xi_{\mathcal{G}}^{X}=\left(\mathcal{C}_{\mathcal{G}}^{X}, p_{u}, U_{\mathcal{G}}^{X}\right)$. Here, $p_{u}=\left(p_{X}\right)_{\mathcal{G}}$.

Theorem 4.2.1 Let $\mathcal{R}^{4}$ be the set of rank 4 nonhyperelliptic Riemann surfaces of genus 4 and $X, \mathcal{G}$ as above. Then $\xi_{\mathcal{G}}^{X}=\left(\mathcal{C}_{\mathcal{G}}^{X}, p_{u}, U_{\mathcal{G}}^{X}\right)$ is a universal $\mathcal{R}^{4}$-family. We denote by $\rho_{u}: \pi_{1}\left(U_{\mathcal{G}}^{X}\right) \rightarrow \Gamma_{4}$ the topological monodromy of $p_{u}: \mathcal{C}_{\mathcal{G}}^{X} \rightarrow U_{\mathcal{G}}^{X}$. Then there exists a unique $\mathbb{Q}$-valued 1 -cochain $\phi_{\mathcal{R}^{4}}: \pi_{1}\left(U_{\mathcal{G}}^{X}\right) \rightarrow \mathbb{Q}$ whose coboundary equals $\rho_{u}^{*} \tau_{4}$. 
Proof We only have to prove the latter part. Consider the map $U_{\mathcal{G}}^{X} \rightarrow B \mathcal{G}$ induced from the projection $E \mathcal{G} \rightarrow B \mathcal{G}$. This is a $U^{X}$-bundle. By the homotopy exact sequence, we have the exact sequence

$$
\pi_{1}(\mathcal{G}) \rightarrow \pi_{1}\left(U^{X}\right) \stackrel{i}{\rightarrow} \pi_{1}\left(U_{\mathcal{G}}^{X}\right) \rightarrow \pi_{0}(\mathcal{G}) \rightarrow *,
$$

where $i$ is induced from the inclusion. But $\pi_{1}(\mathcal{G}) \cong \mathbb{Z} / 2 \mathbb{Z} \oplus \mathbb{Z} / 2 \mathbb{Z}$ and $\pi_{0}(\mathcal{G})$ consists of two points, since $\mathcal{G}$ is isomorphic to the semidirect product $(\operatorname{PGL}(2) \times \operatorname{PGL}(2)) \ltimes$ $\mathbb{Z} / 2 \mathbb{Z}$. So (4.2.2) shows that $i^{*}: H^{2}\left(\pi_{1}\left(U_{\mathcal{G}}^{X}\right) ; \mathbb{Q}\right) \rightarrow H^{2}\left(\pi_{1}\left(U^{X}\right) ; \mathbb{Q}\right)$ is injective. By Theorem 3.1.1, we have $i^{*} \rho_{u}^{*}\left[\tau_{4}\right]=0 \in H^{2}\left(\pi_{1}\left(U^{X}\right)\right.$; $\left.\mathbb{Q}\right)$. Therefore we also have $\rho_{u}^{*}\left[\tau_{4}\right]=0 \in H^{2}\left(\pi_{1}\left(U_{\mathcal{G}}^{X}\right) ; \mathbb{Q}\right)$. This shows the existence of $\phi_{\mathcal{R}^{4}}$.

On the other hand, in the proof of Lemma 3.3.1 we have seen that the abelianization of $\pi_{1}\left(U^{X}\right)$ is a cyclic group of finite order. Combining this fact with (4.2.2), we see that the abelianization of $\pi_{1}\left(U_{\mathcal{G}}^{X}\right)$ is a finite abelian group. This shows the uniqueness of $\phi_{\mathcal{R}^{4}}$.

Combining this with Proposition 4.1.8, we have:

Corollary 4.2.3 Let $\mathcal{R}^{4}$ be the set of rank 4 nonhyperelliptic Riemann surfaces of genus 4 . Then the formula

$$
\sigma_{\mathcal{R}^{4}}(\mathcal{F})=\phi_{\mathcal{R}^{4}}\left(x_{\mathcal{F}}\right)+\operatorname{Sign}(S)
$$

for $\mathcal{F}=(S, \pi, \Delta, b) \in \mathcal{R}_{\text {loc }}^{4}$ (see (4.1.9)) gives a local signature with respect to $\mathcal{R}^{4}$.

\subsection{Some computations of $\sigma_{\mathcal{R}^{4}}$ and $\phi_{\mathcal{R}^{4}}$}

In this section we compute the value of our $\sigma_{\mathcal{R}^{4}}$ or $\phi_{\mathcal{R}^{4}}$ for some examples.

Lemma 4.3.1 Let $X=s_{3,3}\left(\mathbb{P}_{1} \times \mathbb{P}_{1}\right)$ be as defined in Section 4.2. Then $D_{X}$ is a hypersurface and $\operatorname{deg} D_{X}=34$. For a lasso $\sigma_{X}$ around $D_{X}$, we have $\phi_{X}\left(\sigma_{X}\right)=$ $-9 / 17$.

Proof Since $X \cong \mathbb{P}_{1} \times \mathbb{P}_{1}, X$ is simply connected and we have Sign $X=0, \chi(X)=4$. Also we have $\operatorname{deg} X=18$. By Proposition 3.5.3 and (3.5.6), the assertion follows.

Let $\iota: \Delta \rightarrow \mathbb{P}\left(V_{3,3}\right)$ be as in Proposition 2.3.8. Then we get a $\mathcal{R}^{4}$-degeneration $\iota^{*} \mathcal{W} \rightarrow \Delta$ (see Theorem 2.3.12), which we denote by $\mathcal{F}_{I}$ and call a singular fiber germ of type $I$. In this case we can choose $x_{\mathcal{F}_{I}}$ in (4.2.4) to be the image of a lasso around $D_{X}$. By Lemma 3.5.2 the signature of the fiber neighborhood is 0 . Thus we have: 
Proposition 4.3.2 $\sigma_{\mathcal{R}^{4}}\left(\mathcal{F}_{I}\right)=\phi_{\mathcal{R}^{4}}\left(x_{\mathcal{F}_{I}}\right)=-9 / 17$.

In the following let $\Delta=\{z \in \mathbb{C}:|z| \leq \varepsilon\}$ for a sufficiently small real number $\varepsilon>0$.

\section{Example 4.3.3 Let}

$$
\Phi\left(z, a_{0}, a_{1}, b_{0}, b_{1}\right)=\varphi^{0}\left(a_{0}, a_{1}\right) b_{0}{ }^{3}+\left(a_{0}{ }^{3}+z^{6} a_{1}{ }^{3}\right) b_{0} b_{1}{ }^{2}+z^{9} \varphi^{3}\left(a_{0}, a_{1}\right) b_{1}{ }^{3}
$$

and $S_{\Phi} \subset \Delta \times \mathbb{P}_{1} \times \mathbb{P}_{1}$ be the zero locus of $\Phi$. Here $\varphi^{0}, \varphi^{3}$ are generic homogeneous polynomials of degree 3. Let $f^{\prime}: S_{\Phi} \rightarrow \Delta$ be the first projection.

The zero locus $S_{\Phi}$ has an isolated singularity at $(0,[0: 1],[0: 1])$. Applying the resolution process given by Ashikaga [1], we will obtain a resolution $\varpi: \widetilde{S}_{\Phi} \rightarrow S_{\Phi}$ of the singularity. By successive blow down of $(-1)$-curves contained in the fiber at 0 , we finally get the fiber germ what we want.

In the below we describe the resolution process. We shall introduce the inhomogeneous coordinates $a=a_{0} / a_{1}$ and $b=b_{0} / b_{1}$.

First we recall a terminology from [1]. Let $W$ be a complex manifold of dimension two and $L$ a holomorphic line bundle on $W$. Let $\bar{L}=\mathbb{P}\left(\mathcal{O}_{W} \oplus \mathcal{O}_{W}(L)\right)$ the associated $\mathbb{P}_{1}$ bundle. This is the $\mathbb{P}_{1}$ bundle on $W$ whose fiber at $w \in W$ is the projectivization of the dual space of $\mathbb{C} \oplus L_{w}$. Here $L_{w}$ is the fiber of $L \rightarrow W$ at $w$. Let $T=\mathcal{O}_{\bar{L}}(1)$. This is the line bundle on $\bar{L}$ whose fiber at $\bar{\ell}$ (which is a line of $\left(\mathbb{C} \oplus L_{w}\right)^{*}$ for some $w \in W$ ) is the dual space of $\bar{\ell}$. Let $S$ be an irreducible reduced divisor on $\bar{L}$ which is linearly equivalent to $3 T$. In [1], the triple $(S, W, L)$ is called a triple section surface.

Let $W=\Delta \times \mathbb{P}_{1}$ with $\left(z,\left[a_{0}: a_{1}\right]\right)$ the global coordinates, and $L$ a trivial line bundle on $W$. We can introduce the homogeneous fiber coordinates $\left[b_{0}: b_{1}\right]$ for $\bar{L}$ by assigning the linear functional on $\mathbb{C} \oplus L_{w}=\mathbb{C} \oplus \mathbb{C}$, given by $\left(c_{0}, c_{1}\right) \mapsto b_{0} c_{0}+b_{1} c_{1}$, to $\left(b_{0}, b_{1}\right)$. Our $S_{\Phi}$, which is the zero locus of $\Phi$, is naturally identified with an irreducible reduced divisor on $\bar{L}$. Then $\left(S_{\Phi}, W, L\right)$ is a triple section surface in the above sense.

Let $\tau_{1}: W_{1} \rightarrow W$ be the blow up at the origin $p_{1}=(0,[0: 1]) \in W$ and let $\hat{\tau}_{1}=$ $\left(\overline{\tau_{1}}, \tau_{1}\right):\left(S_{1}, W_{1}, L_{1}\right) \rightarrow\left(S_{\Phi}, W, L\right)$ be the triplet blow-up at $p_{1}$ with $\ell_{1}=1$, in the sense of [1, page 181], $W_{1}$ is covered by the two coordinate neighborhoods $U_{z}=\{(z, \tilde{a})\}$ and $U_{a}=\{(\widetilde{z}, a)\}$, and $\tau_{1}$ is given by $\tau_{1}(z, \tilde{a})=(z, \tilde{a} z)$ on $U_{z}$, and $\tau_{1}(\widetilde{z}, a)=(a \tilde{z}, a)$ on $U_{a}$. Note that $L_{1}=\tau_{1}^{*} L-E_{1}$ where $E_{1}$ is the exceptional curve of $\tau_{1}$.

Next, let $p_{2}=(0,0) \in U_{z}$ and let $\tau_{2}: W_{2} \rightarrow W_{1}$ be the blow up at $p_{2}$. Let $\hat{\tau}_{2}=$ $\left(\overline{\tau_{2}}, \tau_{2}\right):\left(S_{2}, W_{2}, L_{2}\right) \rightarrow\left(S_{1}, W_{1}, L_{1}\right)$ be the triplet blow-up at $p_{2}$ with $\ell_{2}=2$. $\left(S_{2}, W_{2}, L_{2}\right)$ is also a triple section surface. Note that $L_{2}=\tau_{2}^{*} L_{1}-2 E_{2}$ where $E_{2}$ is the exceptional curve of $\tau_{2}$. 


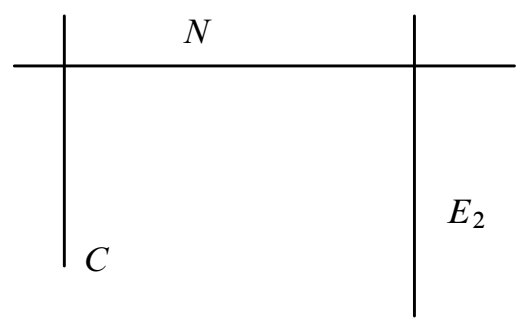

Figure 1

There is a natural map $W_{2} \rightarrow W \rightarrow \Delta$, whose fiber at $0 \in \Delta$ looks like Figure 1. Here, $N$ is the proper transform of $E_{1} \subset W_{1}$, and $C$ is the proper transform of $\{z=0\} \subset W$. All the irreducible components are curves of genus 0 . We denote by $\hat{\pi}_{2}$ the natural projection $S_{2} \rightarrow W_{2}$ and let $N^{*}=\hat{\pi}_{2}^{-1}(N) . N^{*} \subset \bar{L}_{2}$ is a curve of genus 0 .

Let $\bar{\sigma}: M \rightarrow \bar{L}_{2}$ be the blow up with center $N^{*}$ and let $\widetilde{S}_{\Phi}$ be the proper transform of $S_{2}$ by $\bar{\sigma}$ (see [1, page 187]). Then $\widetilde{S}_{\Phi}$ turns out to be nonsingular. Setting $\varpi$ to be the natural map from $\widetilde{S}_{\Phi}$ to $S_{\Phi}$, we get a resolution $\varpi: \widetilde{S}_{\Phi} \rightarrow S_{\Phi}$.

The fiber $\left(f^{\prime} \circ \varpi\right)^{-1}(0) \subset \widetilde{S}_{\Phi}$ looks like Figure 2. Here $C_{1}, N_{1}, N_{2}$ are curves of

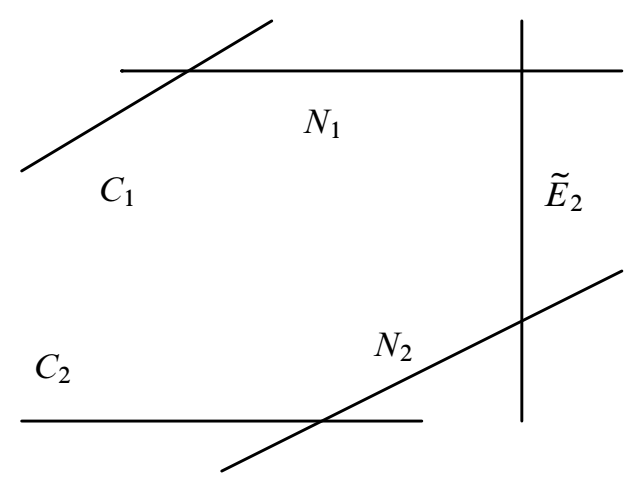

Figure 2

genus $0, C_{2}$ is a curve of genus 1 , and $\widetilde{E}_{2}$ is a curve of genus 3 . The self intersection numbers are: $C_{1}{ }^{2}=N_{2}{ }^{2}=-1, N_{1}{ }^{2}=C_{2}{ }^{2}=-2$, and $\widetilde{E}_{2}^{2}=-3$. The inverse images of $C, N$, and $E_{2}$ by the natural map $\widetilde{S}_{\Phi} \rightarrow W_{2}$ are $C_{1} \amalg C_{2}, N_{1} \amalg N_{2}$, and $\widetilde{E}_{2}$, respectively.

Note that we have a triple covering $\widetilde{S}_{\Phi} \rightarrow W_{2}$. The restrictions of this map to $C_{2}$ or $\widetilde{E}_{2}$ gives a double covering $C_{2} \rightarrow C$ with 4 simple branch points or a triple 
covering $\widetilde{E}_{2} \rightarrow E_{2}$ with 10 simple branch points. As a divisor, $\left(f^{\prime} \circ \varpi\right)^{-1}(0)=$ $C_{1}+N_{1}+\widetilde{E}_{2}+2 N_{2}+C_{2}$.

Finally let $\widetilde{S}_{\Phi} \rightarrow \bar{S}_{\Phi}$ be the contraction obtained by repeating blow down of $(-1)$ curves in the fiber at $0 \in \Delta$ until the resulting surface contains no more such curves (we need to blow down three times). Let $\bar{C}_{2}$ (resp. $\bar{E}_{2}$ ) be the image of $C_{2}$ (resp. $\widetilde{E}_{2}$ ) by this contraction. They are curves of genus 1 and 3 respectively, and $\bar{C}_{2} \cdot \bar{E}_{2}=1$, $\bar{C}_{2}^{2}=\bar{E}_{2}^{2}=-1$.

Let $f_{\Phi}: \bar{S}_{\Phi} \rightarrow \Delta$ be the map induced from $f^{\prime} \circ \varpi$. Then $\mathcal{F}_{3,1}:=\left(\bar{S}_{\Phi}, f_{\Phi}, \Delta, 0\right)$ is a $\mathcal{R}^{4}$-degeneration with $f_{\Phi}^{-1}(0)$ being homeomorphic to the one point union of a surface of genus 1 and a surface of genus 3 .

\section{Proposition 4.3.4 $\sigma_{\mathcal{R}^{4}}\left(\mathcal{F}_{3,1}\right)=11 / 17$ and $\phi_{\mathcal{R}^{4}}\left(x_{\mathcal{F}_{3,1}}\right)=28 / 17$.}

Proof The idea is to globalize the fiber germ $\mathcal{F}_{3,1}$ by a small perturbation. As a result we will obtain a $\mathcal{R}^{4}$-fibration such that the set of singular fiber germs consists of one $\mathcal{F}_{3,1}$ and $\mathcal{F}_{I}$ 's. Then we can compute the number of singular fiber germs, and get the value of $\sigma_{\mathcal{R}^{4}}\left(\mathcal{F}_{3,1}\right)$ by the global signature formula (4.1.7).

Let $\alpha \gg 0$ be a sufficiently large natural number and let $\varphi=\varphi\left(a_{0}, a_{1}, b_{0}, b_{1}\right)$ be a generic $(3,3)$ homogeneous polynomial. Set $\Phi^{\prime}=\Phi+z^{\alpha} \varphi$. Regarding $z$ as an affine coordinate of $\mathbb{P}_{1}$, let $S_{\Phi^{\prime}} \subset \mathbb{P}_{1} \times \mathbb{P}_{1} \times \mathbb{P}_{1}$ be the zero locus of $\Phi^{\prime}$.

The zero locus $S_{\Phi^{\prime}}$ has an isolated singularity at $(0,[0: 1],[0: 1])$, and the same resolution process as that of $S_{\Phi}$ can be applied. Let $\widetilde{S}_{\Phi^{\prime}}$ and $\bar{S}_{\Phi^{\prime}}$ be the result of the process corresponding to $\widetilde{S}_{\Phi}$ and $\bar{S}_{\Phi}$, respectively. Then $\tilde{S}_{\Phi^{\prime}}$ is a nonsingular compact complex surface, and the induced projection $f_{\Phi^{\prime}}: \bar{S}_{\Phi^{\prime}} \rightarrow \mathbb{P}_{1}$ is a $\mathcal{R}^{4}$-fibration. The singular fiber germ at 0 is $\mathcal{F}_{3,1}$, and since $\psi$ is chosen to be generic, the other singular fiber germs is of type $I$.

We compute the various invariants. First of all, the holomorphic Euler characteristic of $S_{\Phi^{\prime}}$ is computed as $\chi\left(\mathcal{O}_{S_{\Phi^{\prime}}}\right)=4 \alpha-3$, and the self intersection number of the dualizing sheaf (see Introduction) of $S_{\Phi^{\prime}}$ is computed as $\omega_{S_{\Phi^{\prime}}}{ }^{2}=14 \alpha-24$. By using Lemma 1.10 and the formula in [1, page 187], we have $\chi\left(\mathcal{O}_{\Phi_{\Phi^{\prime}}}\right)=4 \alpha-10$ and $\omega \widetilde{S}_{\Phi^{\prime}}{ }^{2}=14 \alpha-49$. Thus, we have $\chi\left(\mathcal{O} \bar{S}_{\Phi^{\prime}}\right)=4 \alpha-10$ and $\omega{\overline{S_{\Phi^{\prime}}}}^{2}=14 \alpha-46$. By the Noether formula and the Hirzebruch signature formula, we have $\chi\left(\bar{S}_{\Phi^{\prime}}\right)=34 \alpha-74$ and $\operatorname{Sign}\left(\bar{S}_{\Phi^{\prime}}\right)=-18 \alpha+34$.

As (3.5.6), the number of singular fiber germs of $f_{\Phi^{\prime}}: \bar{S}_{\Phi^{\prime}} \rightarrow \mathbb{P}_{1}$ is computed as

$$
\chi\left(\bar{S}_{\Phi^{\prime}}\right)-2 \cdot(2-2 \cdot 4)=34 \alpha-62 .
$$


In particular, the number of fiber germs of type I is $34 \alpha-63$. By the global signature formula, we have

$$
\operatorname{Sign}\left(\bar{S}_{\Phi^{\prime}}\right)=-18 \alpha+34=(34 \alpha-63) \cdot(-9 / 17)+\sigma_{\mathcal{R}^{4}}\left(\mathcal{F}_{3,1}\right) .
$$

Finally, since the signature of $\bar{S}_{\Phi}$ is $-1, \phi_{\mathcal{R}^{4}}\left(\mathcal{F}_{3,1}\right)=\sigma_{\mathcal{R}^{4}}\left(\mathcal{F}_{3,1}\right)-1$. This completes the proof.

\section{Example 4.3.5 Let}

$\Phi\left(z, a_{0}, a_{1}, b_{0}, b_{1}\right)=\varphi^{0}\left(a_{0}, a_{1}\right) b_{0}{ }^{3}+\varphi^{1}\left(a_{0}, a_{1}\right) b_{0}{ }^{2} b_{1}+a_{0}{ }^{3} b_{0} b_{1}{ }^{2}+z^{6} \varphi^{3}\left(a_{0}, a_{1}\right) b_{1}{ }^{3}$ and $S_{\Phi} \subset \Delta \times \mathbb{P}_{1} \times \mathbb{P}_{1}$ be the zero locus of $\Phi$. Here $\varphi^{0}, \varphi^{1}$ and $\varphi^{3}$ are generic homogeneous polynomials of degree 3 .

$S_{\Phi}$ has an isolated singularity at $(0,[0: 1],[0: 1])$. If we write $\varphi^{i}(a)$ or $\Phi(z, a, b)$ instead of $\varphi^{i}(a, 1)$ or $\Phi(z, a, 1, b, 1)$ respectively, then

$$
\Phi(z, a, b)=\left(\varphi^{0}(a) b+\varphi^{1}(a)\right)\left(b^{2}+A^{3} b+Z^{6}\right),
$$

where $A=a\left(\varphi^{0}(a) b+\varphi^{1}(a)\right)^{-1 / 3}$ and $Z=z\left\{\varphi^{3}(a) /\left(\varphi^{0}(a) b+\varphi^{1}(a)\right)\right\}^{1 / 6}$. Note that

$$
b^{2}+a^{3} b+z^{6}=\left(b+\frac{a^{3}}{2}\right)^{2}-\frac{a^{6}}{4}+Z^{6} .
$$

Set $h(b, z, a)=b^{2}+z^{6}-a^{6}$ and let $S_{h} \subset \mathbb{C}^{3}$ be the zero locus of $h$. Define $f^{\prime}: S_{h} \rightarrow \Delta$ by $f^{\prime}(b, z, a)=z$. Then the singularity of $S_{\Phi}$ is analytically equivalent to the hypersurface singularity $\left(S_{h}, 0\right)$. In this case Horikawa's canonical resolution for double coverings (see [13, Section 2]) can be used for a resolution of $\left(S_{h}, 0\right)$.

The process is as follows. Let $W$ be an open neighborhood of 0 in $\mathbb{C}^{2}$ with global coordinates $(z, a)$ and let $\tau: \widetilde{W} \rightarrow W$ be the blow up at the origin. $\widetilde{W}$ is covered by the two coordinate neighborhoods $U_{z}$ and $U_{a}$ as in Example 4.3.3, and $\tau$ is given by the same formula as $\tau_{1}$. The picture of $\widetilde{W}$ is like Figure 3 . Here $E$ is the exceptional

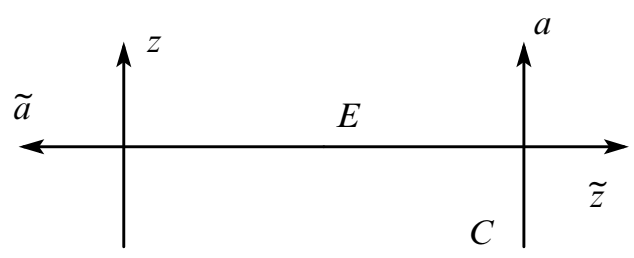

Figure 3

curve of $\tau$ and $C$ is the proper transform of $\{z=0\} \subset W$. 
Let $\widetilde{S}_{h}$ be the double covering on $\widetilde{W}$ defined by the following: on $U_{z}$, let $\widetilde{S}_{h}^{z}$ be the hypersurface in $\mathbb{C}^{3}$ given by $x^{2}+1-\widetilde{a}^{6}=0$ and on $U_{a}$, let $\widetilde{S}_{h}^{a}$ be given by $x^{2}+\widetilde{z}^{6}-1=0$. Identifying $\widetilde{S}_{h}^{z}$ with $\widetilde{S}_{h}^{a}$ over $U_{z} \cap U_{a}$ by the map $(x, z, \tilde{a}) \mapsto$ $\left(x / \widetilde{a}^{3}, \tilde{z}, a\right)$, we get the resulting nonsingular surface $\widetilde{S}_{h}$. Define $\varpi: \widetilde{S}_{h} \rightarrow S_{h}$ by $\varpi(x, z, \tilde{a})=\left(x z^{3}, z, \tilde{a}\right)$ on $\widetilde{S}_{h}^{z}$, and $\varpi(x, \tilde{z}, a)=\left(x a^{3}, a \tilde{z}, a\right)$ on $\tilde{S}_{h}^{a}$. Then this is a resolution of singularity. $\left(f^{\prime} \circ \varpi\right)^{-1}(0)$ looks like in Figure 4.

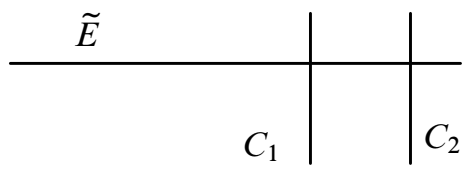

Figure 4

Here $\widetilde{E}$ (resp. $C_{1} \amalg C_{2}$ ) is the proper transform of $E$ (resp. $C$ ). $\widetilde{E}$ is a curve of genus 2. Note that the restriction of $\widetilde{S}_{h} \rightarrow \widetilde{W}$ to $\widetilde{E}$ gives a double covering $\widetilde{E} \rightarrow E \cong \mathbb{P}_{1}$ with 6 simple branch points.

Now applying the above process, we can resolve the singularity of $S_{\Phi}$. Let $\varpi: \widetilde{S}_{\Phi} \rightarrow$ $S_{\Phi}$ be such a resolution. Then $\left(f^{\prime} \circ \varpi\right)^{-1}(0)$ consists of the three components, $\tilde{E}$, $C_{1}$ and $C_{2}$, like Figure 4 (using the same letter). Note that

$$
\Phi(0, a, b)=b\left(\varphi^{0}(a) b^{2}+\varphi^{1}(a) b+a^{3}\right) .
$$

We assume that $C_{1}$ (resp. $C_{2}$ ) is the component corresponding to $b=0$ (resp. $\left.\varphi^{0}(a) b^{2}+\varphi^{1}(a) b+a^{3}=0\right) . C_{1}$ is a curve of genus 0 and $C_{2}$ is a curve of genus 2 . The self intersection numbers are: $\widetilde{E}^{2}=-2, C_{1}{ }^{2}=-1, C_{2}{ }^{2}=-1$.

Let $\widetilde{S}_{\Phi} \rightarrow \bar{S}_{\Phi}$ be the blow down of $\bar{C}_{1}$ and $f_{\Phi}: \bar{S}_{\Phi} \rightarrow \Delta$ the map induced from $f^{\prime} \circ \varpi$. Then $\mathcal{F}_{2,2}:=\left(\bar{S}_{\Phi}, f_{\Phi}, \Delta, 0\right)$ is a $\mathcal{R}^{4}$-degeneration with $f_{\Phi}^{-1}(0)$ being homeomorphic to the one points union of two curves of genus 2 .

Proposition 4.3.6 $\sigma_{\mathcal{R}^{4}}\left(\mathcal{F}_{2,2}\right)=19 / 17$ and $\phi_{\mathcal{R}^{4}}\left(x_{\mathcal{F}_{2,2}}\right)=36 / 17$.

Proof The idea of proof is the same as Proposition 4.3.4. Let $\alpha, \varphi, \Phi^{\prime}$, and $S_{\Phi^{\prime}}$ be the same as in the proof of Proposition 4.3.4. We construct $\widetilde{S}_{\Phi^{\prime}}$ and $f_{\Phi^{\prime}}: \bar{S}_{\Phi^{\prime}} \rightarrow \mathbb{P}_{1}$ by a similar manner to Proposition 4.3.4 except for using the resolution of $\left(S_{h}, 0\right)$ described as above.

We have $\chi\left(\mathcal{O}_{S_{\Phi^{\prime}}}\right)=4 \alpha-3$, and $\omega_{S_{\Phi^{\prime}}}{ }^{2}=14 \alpha-24$. Using Lemma 6 of [13], we have $\chi\left(\mathcal{O} \widetilde{S}_{\Phi^{\prime}}\right)=4 \alpha-6$, and $\omega \widetilde{S}_{\Phi^{\prime}}{ }^{2}=14 \alpha-32$. Thus we have $\chi\left(\mathcal{O}_{\bar{S}_{\Phi^{\prime}}}\right)=4 \alpha-6$ 
and $\omega \bar{S}_{\Phi^{\prime}}{ }^{2}=14 \alpha-31$, therefore $\chi\left(\bar{S}_{\Phi^{\prime}}\right)=34 \alpha-41$ and $\operatorname{Sign}\left(\bar{S}_{\Phi^{\prime}}\right)=-18 \alpha+17$. Now the number of singular fiber germs of $f_{\Phi^{\prime}}$ is

$$
34 \alpha-41-2 \cdot(2-2 \cdot 4)=34 \alpha-29,
$$

hence the number of singular fiber germs of type I is $34 \alpha-30$. By the global signature formula we have

$$
-18 \alpha+17=(34 \alpha-30) \cdot(-9 / 17)+\sigma_{\mathcal{R}^{4}}\left(\mathcal{F}_{2,2}\right) .
$$

Finally the signature of $\bar{S}_{\Phi}$ is -1 . This completes the proof.

\section{Example 4.3.7 Let}

$$
\Phi\left(z, a_{0}, a_{1}, b_{0}, b_{1}\right)=\left(a_{0} b_{1}-a_{1} b_{0}\right)^{3}+z^{3} \varphi^{0}\left(a_{0}, a_{1}, b_{0}, b_{1}\right) .
$$

We write $Y=\Delta \times \mathbb{P}_{1} \times \mathbb{P}_{1}$ and let $S_{\Phi} \subset Y$ be the zero locus of $\Phi$. Here $\varphi^{0} \in V_{3,3}$ is a generic $(3,3)$ homogeneous polynomial.

Let $\Gamma=\{z=0\} \subset Y$ and we denote by $D$ the fiber at 0 of the first projection $S_{\Phi} \rightarrow \Delta$. The fiber $D$ is the diagonal locus in $\Gamma \cong \mathbb{P}_{1} \times \mathbb{P}_{1}$. Let $\tau_{1}: Y_{1} \rightarrow Y$ be the blow up along $D$. Let $E_{0} \subset Y_{1}$ be the proper transform of $\Gamma, E_{1}$ the exceptional set of $\tau_{1}$, and $\tilde{S}_{\Phi} \subset Y_{1}$ the proper transform of $S_{\Phi}$. Note that $E_{1}$ is isomorphic to the Hirzebruch surface $\mathbb{F}_{2}$ of degree 2 .

Then we see that $\widetilde{S}_{\Phi}$ is nonsingular and $\widetilde{S}_{\Phi} \cap E_{0}=\varnothing$. We write $f_{\Phi}: \widetilde{S}_{\Phi} \rightarrow \Delta$ the natural projection. Then we have a $\mathcal{R}^{4}$-degeneration $\mathcal{F}_{R}^{\prime}:=\left(\tilde{S}_{\Phi}, f_{\Phi}, \Delta, 0\right)$. We see that $f_{\Phi}^{-1}(0)=E_{1} \cap S_{1}$ is a smooth curve of genus 4 . This curve is nonhyperelliptic, but not a curve of rank 4 . This can be seen as follows. First we can contract $E_{0}$. Let $\bar{\tau}: Y_{1} \rightarrow \bar{Y}$ be the contraction of $E_{0}$. The projection $Y_{1} \rightarrow \Delta$ induces the projection $\tilde{f}: \bar{Y} \rightarrow \Delta$, whose central fiber $\tilde{f}^{-1}(0)=\bar{\tau}\left(E_{1}\right)$ is $\mathbb{F}_{2}$ and the other fibers are isomorphic to $\mathbb{P}_{1} \times \mathbb{P}_{1}$. Thus, we can think $f_{\Phi}^{-1}(0)$ is contained in $\mathbb{F}_{2}$. Contracting the negative section of $\mathbb{F}_{2}$, we get a quadric $Q_{3}$ of rank 3 in $\mathbb{P}_{3}$. If we map $f_{\Phi}^{-1}(0)$ into $\mathbb{P}_{3}$ by this contraction, then $f_{\Phi}^{-1}(0)$ can be realized as a $(2,3)$-complete intersection: the intersection of $Q_{3}$ and some cubic surface. Thus $f_{\Phi}^{-1}(0)$ is nonhyperelliptic but not of rank 4. Topologically $f_{\Phi}: \widetilde{S}_{\Phi} \rightarrow \Delta$ is a trivial $\Sigma_{4}$-bundle.

Proposition 4.3.8 Let $\mathcal{F}_{R}^{\prime}=\left(\tilde{S}_{\Phi}, f, \Delta, 0\right)$ be the fiber germ as above. Then we have $\sigma_{\mathcal{R}^{4}}\left(\mathcal{F}_{R}^{\prime}\right)=\phi_{\mathcal{R}^{4}}\left(x_{\mathcal{F}_{R}^{\prime}}\right)=4 / 17$.

Proof The proof proceeds as the same before. Let $\alpha, \varphi, \Phi^{\prime}, S_{\Phi^{\prime}}$, and $\widetilde{S}_{\Phi^{\prime}}$ be the same as in the proof of Proposition 4.3.4. We use the same notation $Y$ and $Y_{1}$ for $\mathbb{P}_{1} \times \mathbb{P}_{1} \times \mathbb{P}_{1}$ and the blow up along $D$, respectively. 
First we have $\chi\left(\mathcal{O}_{S_{\Phi^{\prime}}}\right)=4 \alpha-3$ and $\omega_{S_{\Phi^{\prime}}}{ }^{2}=14 \alpha-24$. We need to compute $\chi\left(\mathcal{O} \widetilde{S}_{\Phi^{\prime}}\right)$ and $\omega{\widetilde{S_{\Phi^{\prime}}}}^{2}$. By $K_{Y_{1}} \sim \tau_{1}^{*} K_{Y}+E_{1}$ (linear equivalence) and $S_{1} \sim \tau_{1}^{*} S-3 E_{1}$, we have $K_{Y_{1}}+S_{1} \sim \tau_{1}^{*}\left(K_{Y}+S\right)-2 E_{1}$. By restricting to $S_{1}$, we have $\tau_{1}^{*} \omega_{S}=\omega_{S_{1}}+\left.2 E_{1}\right|_{S_{1}}$. We get

$$
\begin{aligned}
\omega_{S}{ }^{2}=\left(\tau_{1}^{*} \omega_{S}\right)^{2} & =\left(\omega_{S_{1}}+\left.2 E_{1}\right|_{S_{1}}\right)^{2} \\
& =\omega_{S_{1}}{ }^{2}+\left.4 \omega_{S_{1}} \cdot E_{1}\right|_{S_{1}}+4\left(\left.E_{1}\right|_{S_{1}}\right)^{2}=\omega_{S_{1}}{ }^{2}+24,
\end{aligned}
$$

since $\left.\omega_{S_{1}} \cdot E_{1}\right|_{S_{1}}=2 g\left(E_{1} \cap S_{1}\right)-2-\left(\left.E_{1}\right|_{S_{1}}\right)^{2}=6-\left(\left.E_{1}\right|_{S_{1}}\right)^{2}$ by the adjunction formula. Therefore, $\omega{\widetilde{S_{\Phi}^{\prime}}}^{2}=14 \alpha-48$. We next compute

$$
\begin{aligned}
\chi\left(\mathcal{O}_{S_{1}}\right) & =\chi\left(\mathcal{O}_{Y_{1}}\right)-\chi\left(\mathcal{O}_{Y_{1}}\left(-S_{1}\right)\right) \\
& =\chi\left(\mathcal{O}_{Y_{1}}\right)-\chi\left(\mathcal{O}_{Y_{1}}\left(-S_{1}-E_{1}\right)\right)-\chi\left(\mathcal{O}_{E_{1}}\left(-\left.S_{1}\right|_{E_{1}}\right)\right) \\
& \vdots \\
& =\chi\left(\mathcal{O}_{Y_{1}}\right)-\chi\left(\mathcal{O}_{Y_{1}}\left(-S_{1}-3 E_{1}\right)\right)-\sum_{i=0}^{2} \chi\left(\mathcal{O}_{E_{1}}\left(-\left.S_{1}\right|_{E_{1}}-\left.i E_{1}\right|_{E_{1}}\right)\right) .
\end{aligned}
$$

We have $\chi\left(\mathcal{O}_{Y_{1}}\right)-\chi\left(\mathcal{O}_{Y_{1}}\left(-S_{1}-3 E_{1}\right)\right)=\chi\left(\mathcal{O}_{Y_{1}}\right)-\chi\left(\mathcal{O}_{Y_{1}}\left(-\tau_{1}^{*} S\right)\right)=\chi\left(\mathcal{O}_{Y}\right)-$ $\chi\left(\mathcal{O}_{Y}(-S)\right)=\chi\left(\mathcal{O}_{S}\right)$. To compute the remaining term, we use

$$
\chi\left(\mathcal{O}_{E_{1}}\left(-\left.S_{1}\right|_{E_{1}}-\left.i E_{1}\right|_{E_{1}}\right)\right)=\chi\left(\mathcal{O}_{E_{1}}\left(-\left.i E_{1}\right|_{E_{1}}\right)\right)-\chi\left(\mathcal{O}_{E_{1} \cap S_{1}}\left(-\left.i E_{1}\right|_{E_{1} \cap S_{1}}\right)\right) .
$$

Note that the divisor $\left.\left(E_{0}+E_{1}\right)\right|_{E_{1}}$ is trivial on $E_{1}$, since $E_{0}+E_{1}$ is a fiber of $Y_{1} \rightarrow \Delta$; $C_{\infty}=E_{0} \cap E_{1}$ is the negative section of $E_{1} \cong \mathbb{F}_{2}$, so $C_{\infty}^{2}=-2 ; E_{0} \cap E_{1} \cap S_{1}=\varnothing$. From these we have $\mathcal{O}_{E_{1}}\left(-\left.E_{1}\right|_{E_{1}}\right) \cong \mathcal{O}_{E_{1}}\left(C_{\infty}\right)$ and $\mathcal{O}_{E_{1} \cap S_{1}}\left(-E_{1}\right) \cong \mathcal{O}_{E_{1} \cap S_{1}}$. Using the Riemann-Roch formula and $\chi\left(\mathcal{O}_{E_{1}}\right)=1$, we get $\chi\left(\mathcal{O}_{E_{1}}\left(-S_{1} \mid E_{1}\right)\right)=4$; $\chi\left(\mathcal{O}_{E_{1}}\left(-\left.S_{1}\right|_{E_{1}}-\left.E_{1}\right|_{E_{1}}\right)\right)=3 ; \chi\left(\mathcal{O}_{E_{1}}\left(-\left.S_{1}\right|_{E_{1}}-\left.2 E_{1}\right|_{E_{1}}\right)\right)=0$.

In summary, we have $\chi\left(\mathcal{O}_{S_{1}}\right)=\chi\left(\mathcal{O}_{S}\right)-7$. Therefore, $\chi\left(\mathcal{O}_{\widetilde{S}_{\Phi^{\prime}}}\right)=4 \alpha-10$.

Now $\chi\left(\widetilde{S}_{\Phi^{\prime}}\right)=34 \alpha-72$, and $\operatorname{Sign}\left(\widetilde{S}_{\Phi^{\prime}}\right)=-18 \alpha+32$. The number of topologically singular fibers is

$$
34 \alpha-72-2 \cdot(2-2 \cdot 4)=34 \alpha-60 .
$$

By the global signature formula we have

$$
-18 \alpha+32=(34 \alpha-60) \cdot(-9 / 17)+\sigma_{\mathcal{R}^{4}}\left(\mathcal{R}_{3}\right) .
$$

Finally, the signature of $\widetilde{S}_{\Phi}$ is zero. This completes the proof.

Example 4.3.9 In this last example we do not use the global signature formula (4.1.7) directly. Let $q_{1}=x_{0}{ }^{2}+x_{1}{ }^{2}+x_{3}{ }^{2}$ and $q_{2}=x_{1}{ }^{2}+x_{2}{ }^{2}-x_{3}{ }^{2}$, and $h\left(x_{0}, x_{1}, x_{2}, x_{3}\right)$ a generic cubic polynomial. Let

$$
S_{h}=\left\{(x, z) \in \mathbb{P}_{3} \times \Delta: h(x)=\left(q_{1}+z q_{2}\right)(x)=0\right\},
$$


and let $f: S_{h} \rightarrow \Delta$ be the second projection. Note that $q_{1}+z q_{2}$ defines a smooth quadric except for $z=0$ and $f^{-1}(0)$ is contained in the singular quadric $\left\{q_{1}=0\right\}$. Thus $\mathcal{F}_{R}:=\left(S_{h}, f, \Delta, 0\right)$ is a $\mathcal{R}^{4}$-degeneration. Topologically $f: S_{h} \rightarrow \Delta$ is a trivial $\Sigma_{4}$-bundle.

Proposition 4.3.10 $\sigma_{\mathcal{R}^{4}}\left(\mathcal{F}_{R}\right)=\phi_{\mathcal{R}^{4}}\left(x_{\mathcal{F}_{R}}\right)=2 / 17$.

Proof We start from describing the associated principal $\mathcal{G}$-bundle $P\left(\xi_{\mathcal{F}_{R}}\right)$.

Let $\omega_{z}$ be the basis of $\Omega^{1}\left(f^{-1}(z)\right) \subset \mathbb{P}_{3}$ corresponding to the homogeneous coordinates of $\mathbb{P}_{3}$ (see the paragraph before Theorem 4.2.1). Any frame of $\Omega^{1}\left(f^{-1}(z)\right.$ ) (modulo $\mathbb{C}^{*}$ ) is written as the form $A \cdot \omega_{z}, A \in \mathrm{PGL}(4)$. Then

$$
\begin{gathered}
P\left(\xi_{\mathcal{F}_{R}}\right) \cong\left\{(z, A) \in(\Delta \backslash\{0\}) \times \operatorname{PGL}(4) ;\left(A^{-1}\right)^{t} B(z) A^{-1}=H\right\}, \\
B(z)=\left(\begin{array}{cccc}
1 & 0 & 0 & 0 \\
0 & 1+z & 0 & 0 \\
0 & 0 & z & 0 \\
0 & 0 & 0 & 1-z
\end{array}\right) .
\end{gathered}
$$

Let $\kappa: \Delta \backslash\{0\} \rightarrow \Delta \backslash\{0\}$ be the map defined by $w \mapsto z^{2}$ and consider the pullback $\kappa^{*} P\left(\xi_{\mathcal{F}_{R}}\right)$. Then the principal $\mathcal{G}$-bundle $\kappa^{*} P\left(\xi_{\mathcal{F}_{R}}\right) \rightarrow \Delta \backslash\{0\}$ has a section given by $w \mapsto(w, A(w))$ where

$$
A(w)=\frac{1}{\sqrt{2}}\left(\begin{array}{cccc}
1 & \sqrt{-1} r_{1}(w) & 0 & 0 \\
0 & 0 & w & \sqrt{-1} r_{2}(w) \\
0 & 0 & -w & \sqrt{-1} r_{2}(w) \\
1 & -\sqrt{-1} r_{1}(w) & 0 & 0
\end{array}\right)
$$

Here, $r_{1}(w)=\sqrt{1+w^{2}}$ and $r_{2}(w)=\sqrt{1-w^{2}}$. Thus, as a candidate for $g_{\kappa} * \mathcal{F}_{R}$ (see the proof of Proposition 4.1.8), we can take the map given by $w \mapsto[e, A(w)$. $\left.f\left(a_{0} b_{0}, a_{0} b_{1}, a_{1} b_{0}, a_{1} b_{1}\right)\right]$. Since the diagram

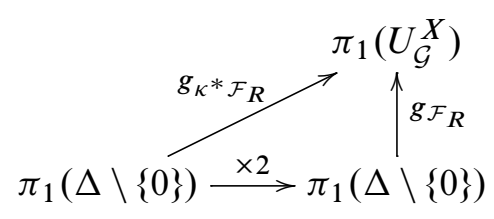

is commutative up to conjugacy, $x_{\mathcal{F}_{R}}{ }^{2}$ is conjugate to $x_{\kappa^{*} \mathcal{F}_{R}}=g_{\kappa^{*} \mathcal{F}_{R}}(\partial \Delta)$.

But now $A(w) \cdot f\left(a_{0} b_{0}, a_{0} b_{1}, a_{1} b_{0}, a_{1} b_{1}\right)$ is equal to

$$
f\left(\frac{a_{0} b_{0}+a_{1} b_{1}}{\sqrt{2}}, \frac{a_{0} b_{0}-a_{1} b_{1}}{\sqrt{-1} r_{1}(w)}, \frac{a_{0} b_{1}-a_{1} b_{0}}{w}, \frac{a_{0} b_{1}+a_{1} b_{0}}{\sqrt{-1} r_{2}(w)}\right) .
$$


Modulo $\mathbb{C}^{*}$ this can be written as

$$
\left(a_{0} b_{1}-a_{1} b_{0}\right)^{3}+w^{3}\left(\varphi^{0}+w \varphi^{1}+\text { higher term with respect to } w\right)
$$

where $\varphi^{i}$ is some $(3,3)$ homogeneous polynomial. This shows that $x_{\kappa^{*}} \mathcal{F}_{R}$ is homotopic to $x_{\mathcal{F}_{R}^{\prime}}$ in Proposition 4.3.8. Since $f: S_{h} \rightarrow \Delta$ is topologically trivial,

$$
4 / 17=\phi_{\mathcal{R}^{4}}\left(x_{\mathcal{F}_{R}^{\prime}}\right)=\phi_{\mathcal{R}^{4}}\left(x_{\kappa^{*} \mathcal{F}_{R}}\right)=\phi_{\mathcal{R}^{4}}\left(x_{\mathcal{F}_{R}}{ }^{2}\right)=2 \phi_{\mathcal{R}^{4}}\left(x_{\mathcal{F}_{R}}\right) .
$$

This completes the proof.

Compare the above proof with [3, Example 7.5], where the same fiber germ is considered.

\subsection{Fibrations of nontrigonal curves of genus 5}

Let $C$ be a nonhyperelliptic Riemann surface of genus 5. By the Enriques-Petri theorem [10, page 535], the canonical image of $C$ is a $(2,2,2)$ complete intersection if and only if $C$ is nontrigonal, ie, there is no holomorphic map $C \rightarrow \mathbb{P}_{1}$ of degree 3. Let $\mathcal{N} \mathcal{T}^{5} \subset \mathbb{M}_{5}$ be the set of nonhyperelliptic and nontrigonal Riemann surface of genus 5. $\mathcal{N} \mathcal{T}^{5}$ is Zariski open in $\mathbb{M}_{5}$.

We denote by $\left[\alpha_{0}: \alpha_{1}: \alpha_{2}: \alpha_{3}: \alpha_{4}\right]$ the homogeneous coordinates of $\mathbb{P}_{4}$ and let $S_{5}$ be the space of $5 \times 5$ symmetric matrices. The Veronese map $v_{2}: \mathbb{P}_{4} \rightarrow \mathbb{P}\left(S_{5}\right)$ is given by

$$
v_{2}\left(\left[\alpha_{0}: \alpha_{1}: \alpha_{2}: \alpha_{3}: \alpha_{4}\right]\right):=\left[\left(\alpha_{0}, \alpha_{1}, \alpha_{2}, \alpha_{3}, \alpha_{4}\right)^{t} \cdot\left(\alpha_{0}, \alpha_{1}, \alpha_{2}, \alpha_{3}, \alpha_{4}\right)\right] .
$$

This map is equivariant with respect to the action of $\mathcal{G}=\operatorname{PGL}(5)$, where the action of $\mathcal{G}$ on $\mathbb{P}_{4}$ is induced by

$$
A \cdot\left(\alpha_{0}, \alpha_{1}, \alpha_{2}, \alpha_{3}, \alpha_{4}\right)=\left(\alpha_{0}, \alpha_{1}, \alpha_{2}, \alpha_{3}, \alpha_{4}\right) A^{t}
$$

for $A \in \mathrm{GL}_{5}(\mathbb{C})$ and $\alpha_{i} \in \mathbb{C}$, and the action of $\mathcal{G}$ on $\mathbb{P}\left(S_{5}\right)$ is induced by $A \cdot B=A B A^{t}$ for $A \in \mathrm{GL}_{5}(\mathbb{C})$ and $B \in S_{5}$. Set

$$
X:=v_{2}\left(\mathbb{P}_{4}\right) .
$$

The action of $\mathcal{G}$ on $G_{11}\left(\mathbb{P}\left(S_{5}\right)\right)$ induced from the $\mathcal{G}$-action on $\mathbb{P}\left(S_{5}\right)$ preserves $D_{X}$ and $U^{X}=G_{11}\left(\mathbb{P}\left(S_{5}\right)\right) \backslash D_{X}$, and the projection $p_{X}: \mathcal{C}^{X} \rightarrow U^{X}$ is $\mathcal{G}$-equivariant. Note that for $W \in U^{X}$, the fiber $p_{X}^{-1}(W)$ is isomorphic to a smooth complete intersection in $\mathbb{P}_{4}$ of type $(2,2,2)$. Thus $\xi^{X}=\left(\mathcal{C}^{X}, p_{X}, U^{X}\right)$ is a $\mathcal{N} \mathcal{T}^{5}$-family.

We claim that $\xi^{X}$ and the $\mathcal{G}$-action on it satisfies the conditions in Proposition 4.1.3. The proof is similar to the case of $\mathcal{R}^{4}$, so we only describe the way of constructing $\mathcal{G}$-bundles and $\mathcal{G}$-equivariant maps. 
Let $C \in \mathcal{N} \mathcal{T}^{5}$. By Max Noether's theorem, the natural map $t_{2}: \operatorname{Sym}^{2} \Omega^{1}(C) \rightarrow$ $H^{0}\left(C ; K_{C}^{\otimes 2}\right)$ is surjective hence the kernel is 3-dimensional. By taking the dual, we get the codimension 3 subspace $\operatorname{Ker}\left(t_{2}\right)^{*} \subset \operatorname{Sym}^{2} \Omega^{1}(C)^{*}$. If we take a basis $\omega=\left(\omega_{0}, \omega_{1}, \omega_{2}, \omega_{3}, \omega_{4}\right)$ of $\Omega^{1}(C), \operatorname{Sym}^{2} \Omega^{1}(C)^{*}$ is identified with $S_{5}$ by assigning $B \in S_{5}$ with the quadratic function

$$
\Omega^{1}(C) \rightarrow \mathbb{C}, x_{0} \omega_{0}+\cdots+x_{4} \omega_{4} \mapsto\left(x_{0}, \ldots, x_{4}\right) B\left(x_{0}, \ldots, x_{4}\right)^{t},
$$

and we have the plane $B(\omega) \subset \mathbb{P}\left(S_{5}\right)$ of codimension 3 corresponding to $\operatorname{Ker}\left(t_{2}\right)^{*}$. Note that the image $v_{2} \circ \iota_{\omega}(C)$, where $\iota_{\omega}: C \rightarrow \mathbb{P}_{4}$ is the canonical map given by $c \mapsto\left[\omega_{0}(c): \cdots: \omega_{4}(c)\right]$, is the smooth intersection of $X$ and $B(\omega)$.

Let $P(C)$ be the set of all frames of $\Omega^{1}(C)$ modulo $\mathbb{C}^{*}$. By assigning $\omega \in P(C)$ with $B(\omega)$, we have the map $E_{C}: P(C) \rightarrow U^{X}$. Moreover, given the left action of $\mathcal{G}$ on $P(C)$ by the same formula as the action of $\mathrm{PO}_{4}^{H}(\mathbb{C})$ on $P(C)$ in Section 4.2, we see that $E_{C}$ is $\mathcal{G}$-equivariant.

Let $\xi=(\mathcal{C}, p, B)$ be a $\mathcal{N} \mathcal{T}^{5}$-family. Applying the above construction to all the fibers, we get a principal $\mathcal{G}$-bundle $P(\xi)$ and a $\mathcal{G}$-equivariant map $E_{\xi}: P(\xi) \rightarrow U^{X}$ what we want.

Theorem 4.4.1 Let $\mathcal{N} \mathcal{T}^{5}$ be the set of nonhyperelliptic and nontrigonal Riemann surfaces of genus 5 and $X, \mathcal{G}$ as above. Then $\xi_{\mathcal{G}}^{X}=\left(\mathcal{C}_{\mathcal{G}}^{X}, p_{u}, U_{\mathcal{G}}^{X}\right)$ is a universal $\mathcal{N T}^{5}$ family. We denote by $\rho_{u}: \pi_{1}\left(U_{\mathcal{G}}^{X}\right) \rightarrow \Gamma_{5}$ the topological monodromy of $p_{u}: \mathcal{C}_{\mathcal{G}}^{X} \rightarrow$ $U_{\mathcal{G}}^{X}$. Then there exists a unique $\mathbb{Q}$-valued 1 -cochain $\phi_{\mathcal{N} \mathcal{T}^{5}}: \pi_{1}\left(U_{\mathcal{G}}^{X}\right) \rightarrow \mathbb{Q}$ whose coboundary equals $\rho_{u}^{*} \tau_{5}$.

Proof The proof is the same as the proof of Theorem 4.2.1 except that (4.2.2) is replaced with the exact sequence

$$
\pi_{1}(\mathcal{G}) \cong \mathbb{Z} / 5 \mathbb{Z} \rightarrow \pi_{1}\left(U^{X}\right) \rightarrow \pi_{1}\left(U_{\mathcal{G}}^{X}\right) \rightarrow * .
$$

Corollary 4.4.2 Let $\mathcal{N} \mathcal{T}^{5}$ be the set of nonhyperelliptic and nontrigonal Riemann surfaces of genus 5 . Then the formula

$$
\sigma_{\mathcal{N} \mathcal{T}^{5}}(\mathcal{F})=\phi_{\mathcal{N} \mathcal{T}^{5}}\left(x_{\mathcal{F}}\right)+\operatorname{Sign}(S)
$$

for $\mathcal{F}=(S, \pi, \Delta, b) \in \mathcal{N} \mathcal{T}_{\text {loc }}^{5}$ (see (4.1.9)) gives a local signature with respect to $\mathcal{N} \mathcal{T}^{5}$.

Lemma 4.4.3 Let $X=v_{2}\left(\mathbb{P}_{4}\right)$ be as above. Then $D_{X}$ is a hypersurface and deg $D_{X}=$ 40. For a lasso $\sigma_{X}$ around $D_{X}$, we have $\phi_{X}\left(\sigma_{X}\right)=-1 / 2$.

Proof This follows from Proposition 3.5.13. 
Let $\iota: \Delta \rightarrow G_{11}\left(\mathbb{P}\left(S_{5}\right)\right)$ be as in Proposition 2.3.8. Then we get a $\mathcal{N} \mathcal{T}^{5}$-degeneration $\iota^{*} \mathcal{W} \rightarrow \Delta$, which we denote by $\mathcal{F}_{I}$ and call a singular fiber germ of type I.

Proposition 4.4.4 Let $\mathcal{F}_{I} \in \mathcal{N} \mathcal{T}_{\text {loc }}^{5}$ be as above. Then

$$
\sigma_{\mathcal{N} \mathcal{T}^{5}}\left(\mathcal{F}_{I}\right)=\phi_{\mathcal{N} \mathcal{T}^{5}}\left(x_{\mathcal{F}_{I}}\right)=-1 / 2
$$

Proof The proof is similar to the proof of Proposition 4.3.2.

This fiber germ is expected to play an important role when computing examples as $\mathcal{F}_{I} \in \mathcal{R}_{\text {loc }}^{4}$ behaves like an "atomic" germ in Section 4.3, but at the present moment we don't have any example of element of $\mathcal{N} \mathcal{T}^{5}$ other than $\mathcal{F}_{I}$ whose local signature has been computed.

Final remarks Although the construction of our local signature is purely topological, we have used some algebraic geometry to compute examples. It is an interesting problem to find and compute examples of fiber germs beyond the reach of algebraic geometry, or to give a formula for the Meyer functions $\phi_{X}$ as Meyer and Atiyah did. To do this we need a greater understanding of $\rho_{X}$ or the topological monodromy $\rho_{u}$ of a universal $\mathcal{A}$-family.

In the case of $\mathcal{A}=\mathcal{R}^{4}$ or $\mathcal{N} \mathcal{T}^{5}, \mathcal{A}$ is Zariski open in the moduli space. Using this, we can prove that $\rho_{u}$ is surjective. The proof is similar to the proof of [16, Proposition 6.3]. Here is an outline. Let $\mathcal{T}_{g}$ be the Teichmüller space of genus $g$, and let $\tilde{\mathcal{A}} \subset \mathcal{T}_{g}$ be the inverse image of $\mathcal{A}$ by the quotient map $\mathcal{T}_{g} \rightarrow \mathbb{M}_{g}$. Then $\tilde{\mathcal{A}}$ is Zariski open hence path connected, and is preserved by the action of $\Gamma_{g}$ on $\mathcal{T}_{g}$. By a natural way we get an $\mathcal{A}$-family on the Borel construction $\widetilde{\mathcal{A}}_{\Gamma_{g}}$, which is easily seen to be universal. The homotopy exact sequence

$$
\pi_{1}\left(\tilde{\mathcal{A}}_{\Gamma_{g}}\right) \rightarrow \pi_{1}\left(B \Gamma_{g}\right)=\Gamma_{g} \rightarrow \pi_{0}(\tilde{\mathcal{A}})=*
$$

of the $\widetilde{\mathcal{A}}$-bundle $\widetilde{\mathcal{A}}_{\Gamma_{g}} \rightarrow B \Gamma_{g}$ shows the desired surjectivity.

Acknowledgements I would like to thank Tadashi Ashikaga, who kindly communicated the constructions of the first three examples in Section 4.3 to me. I also would like to thank my advisor Nariya Kawazumi for reading a draft, giving various comments, and a warm encouragement. This research is supported by JSPS Research Fellowships for Young Scientists (19.5472). 


\section{References}

[1] T Ashikaga, Normal two-dimensional hypersurface triple points and the Horikawa type resolution, Tohoku Math. J. (2) 44 (1992) 177-200 MR1161610

[2] T Ashikaga, K Konno, Global and local properties of pencils of algebraic curves, from: "Algebraic geometry 2000, Azumino (Hotaka)", (S Usui, M Green, L Illusie, K Kato, E Looijenga, S Mukai, S Saito, editors), Adv. Stud. Pure Math. 36, Math. Soc. Japan, Tokyo (2002) 1-49 MR1971511

[3] T Ashikaga, K-I Yoshikawa, A divisor on the moduli space of curves associated to the signature of fibered surfaces (with an appendix by Kazuhiro Konno [2604074]), from: "Singularities-Niigata-Toyama 2007”, (J-P Brasselet, S Ishii, T Suwa, M Vaquie, editors), Adv. Stud. Pure Math. 56, Math. Soc. Japan, Tokyo (2009) 1-34 MR2604073

[4] M Atiyah, The logarithm of the Dedekind $\eta$-function, Math. Ann. 278 (1987) 335-380 MR909232

[5] W P Barth, K Hulek, C A M Peters, A Van de Ven, Compact complex surfaces, second edition, Ergebnisse der Math. und ihrer Grenzgebiete. 3. Folge. 4, Springer, Berlin (2004) MR2030225

[6] J A Carlson, D Toledo, Discriminant complements and kernels of monodromy representations, Duke Math. J. 97 (1999) 621-648 MR1682991

[7] I Dolgachev, A Libgober, On the fundamental group of the complement to a discriminant variety, from: "Algebraic geometry (Chicago, Ill., 1980)", (A Libgober, P Wagreich, editors), Lecture Notes in Math. 862, Springer, Berlin (1981) 1-25 MR644816

[8] H Endo, Meyer's signature cocycle and hyperelliptic fibrations, Math. Ann. 316 (2000) 237-257 MR1741270

[9] I M Gel'fand, M M Kapranov, A V Zelevinsky, Discriminants, resultants, and multidimensional determinants, Math.: Theory \& Appl., Birkhäuser, Boston (1994) MR1264417

[10] P Griffiths, J Harris, Principles of algebraic geometry, Wiley Classics Library, Wiley, New York (1994) MR1288523 Reprint of the 1978 original

[11] M Gromov, Volume and bounded cohomology, Inst. Hautes Études Sci. Publ. Math. (1982) 5-99 (1983) MR686042

[12] J Harer, The second homology group of the mapping class group of an orientable surface, Invent. Math. 72 (1983) 221-239 MR700769

[13] E Horikawa, On deformations of quintic surfaces, Proc. Japan Acad. 49 (1973) 377379 MR0330173

[14] S Iida, Adiabatic limits of $\eta$-invariants and the Meyer functions, Math. Ann. 346 (2010) 669-717 MR2578566 
[15] N Katz, Pinceaux de Lefschetz: théoréme d'existence, from: "Groupes de monodromie en géométrie algébrique. II", Lecture Notes in Math. 340, Springer, Berlin (1973) 212-253 MR0354657 Séminaire de Géométrie Algébrique du Bois-Marie 1967-1969 (SGA 7 II)

[16] Y Kuno, The mapping class group and the Meyer function for plane curves, Math. Ann. 342 (2008) 923-949 MR2443768

[17] K Lamotke, The topology of complex projective varieties after S Lefschetz, Topology 20 (1981) 15-51 MR592569

[18] Y Matsumoto, On 4-manifolds fibered by tori, Proc. Japan Acad. Ser. A Math. Sci. 58 (1982) 298-301 MR682687

[19] Y Matsumoto, Lefschetz fibrations of genus two-a topological approach, from: "Topology and Teichmüller spaces (Katinkulta, 1995)", (S Kojima, Y Matsumoto, K Saito, M Seppälä, editors), World Sci. Publ., River Edge, NJ (1996) 123-148 MR1659687

[20] W Meyer, Die Signatur von Flächenbündeln, Math. Ann. 201 (1973) 239-264 MR0331382

[21] E Y Miller, The homology of the mapping class group, J. Differential Geom. 24 (1986) 1-14 MR857372

[22] T Morifuji, On Meyer's function of hyperelliptic mapping class groups, J. Math. Soc. Japan 55 (2003) 117-129 MR1939188

[23] S Morita, Characteristic classes of surface bundles, Invent. Math. 90 (1987) 551-577 MR914849

[24] D Mumford, Algebraic geometry. I. Complex projective varieties, Grund. der Math. Wissenschaften 221, Springer, Berlin (1976) MR0453732

[25] D Mumford, Towards an enumerative geometry of the moduli space of curves, from: “Arithmetic and geometry, Vol. II", (M Artin, J Tate, editors), Progr. Math. 36, Birkhäuser, Boston (1983) 271-328 MR717614

[26] I Shimada, Generalized Zariski-van Kampen theorem and its application to Grassmannian dual varieties, Internat. J. Math. 21 (2010) 591-637 MR2650851

[27] V G Turaev, First symplectic Chern class and Maslov indices, J. Soviet Math. 37 (1987) 1115-1127 MR806563

Department of Mathematics, Graduate School of Science, Hiroshima University 1-3-1 Kagamiyama, Higashi-Hiroshima, 739-8526, Japan

kunotti@hiroshima-u.ac.jp

Received: 6 May 2010 Revised: 2 October 2010 